\title{
GENERALIZED RICCI FLOW II: EXISTENCE FOR COMPLETE NONCOMPACT MANIFOLDS
}

\author{
YI LI
}

\begin{abstract}
In this paper, we continue to study the generalized Ricci flow. We give a criterion on steady gradient Ricci soliton on complete and noncompact Riemannian manifolds that is Ricci-flat, and then introduce a natural flow whose stable points are Ricci-flat metrics. Modifying the argument used by Shi and List, we prove the short time existence and higher order derivatives estimates.
\end{abstract}

\section{Contents}

1. Introduction

1.1. Compact steady gradient Ricci solitons

1.2. Complete noncompact steady Ricci solitons

1.3. A parabolic flow

1.4. Notions and convenience

2. A parabolic geometric flow

21 A characterization of Ricci-flat metrics 6

2.2. Evolution equations 7

$\begin{array}{lll}2.3 . & \text { Regular flows on compact manifolds } & 15\end{array}$

$\begin{array}{lll}2.4 . & \text { Reduction to }\left(\alpha_{1}, 0, \beta_{1}, \beta_{2}\right) \text {-flow } & 17\end{array}$

$\begin{array}{lll}2.5 . & \text { De Turck's trick } & 18\end{array}$

$\begin{array}{lll}3 . & \text { Complete and noncompact case } & 19\end{array}$

3.1. Zeroth order estimates 20

3.2. Existence of the De Turck flow 22

3.3. First order derivative estimates 31

3.4. Second order derivative estimates 32

3.5. Higher order derivatives estimates 58

\begin{tabular}{ll}
\hline References & 62
\end{tabular}

\section{INTRODUCTION}

Ricci-flat metrics play an important role in geometry and physics. For compact Kähler manifold with trivial first Chern class, the existence of a (Kähler) Ricciflat metric was proved by Yau in his famous paper [29] on the Calabi conjecture.

2000 Mathematics Subject Classification. Primary 54C40, 14E20; Secondary 46E25, 20C20.

Key words and phrases. Steady gradient Ricci solitons, geometric flow, Ricci-flat metrics.

Yi Li is partially supported by Shanghai Sailing Program (grant) No. 14YF1401400, NSFC (grant) No. 11401374, and the Fonds National de la Recherche Luxembourg (FNR) unde the OPEN scheme (project GEOMREV O14/7628746). 
In the Riemannian setting, Ricci-flat metrics are stationary solutions of the Ricci flow introduced by Hamilton [13] as a powerful tool, together with Perelman's breakthrough 22, 23, 24, to study the Poincaré conjecture.

In the study of the singularities of the Ricci flow, Ricci solitons naturally arises as the self-similar solutions. From the definition, A Ricci-flat metric is indeed a Ricci soliton.

1.1. Compact steady gradient Ricci solitons. In particular, we consider a steady gradient Ricci solition which is a triple $(M, g, f)$, where $M$ is a smooth manifold, $g$ is a Riemannian metric on $M$ and $f$ is a smooth function, such that

$$
\operatorname{Ric}_{g}+\nabla_{g}^{2} f=0 \text { or } R_{i j}+\nabla_{i} \nabla_{j} f=0 .
$$

Hamilton [15] showed that on a compact manifold any steady gradient Ricci soliton must be Ricci-flat; this, together with Perelman's result 22 that any compact Ricci soliton is necessarily a gradient Ricci soliton, implies that any compact steady Ricci soliton must be Ricci-flat (cf. [5, 22])

1.2. Complete noncompact steady Ricci solitons. Now we suppose $(M, g, f)$ is a complete noncompact steady gradient Ricci soliton. The simplest example is Hamilton's cigar soliton or Witten's black hole ( 9 , 11]), which is the complete Riemann surface $\left(\mathbf{R}^{2}, g_{\mathrm{cs}}\right)$ where

$$
g_{\mathrm{cs}}:=\frac{d x \otimes d x+d y \otimes d y}{1+x^{2}+y^{2}} .
$$

If we define

$$
f(x, y):=-\ln \left(1+x^{2}+y^{2}\right)
$$

then

$$
\operatorname{Ric}_{g_{\mathrm{cs}}}+\nabla_{g_{\mathrm{cs}}}^{2} f=0
$$

The cigar soliton is rotationally symmetric, has positive Gaussian curvature, and is asymptotic to a cylinder near infinity; moreover, up to homothety, the cigar soliton is the uniques rotationally symmetric gradient Ricci soliton of positive curvature on $\mathbf{R}^{2}$ (cf. 9, 11]). The classification of two-dimensional complete compact steady gradient Ricci solitons was achieved by Hamilton [15, which states that Any complete noncompact steady gradient Ricci soliton with positive Gaussian curvature is indeed the cigar soliton.

The cigar soliton can be generalized to a rotationally symmetric steady gradient Ricci soliton in higher dimensions on $\mathbf{R}^{n}$. The resulting solitons are referred to be Bryant's solitons (see [11 for the construction), which is rotationally symmetric and has positive Riemann curvature operator. Other examples of steady gradient Ricci solions were constructed by Cao [4] and Ivey [16.

For three-dimensional case, Perelman 22] conjectured a classification of complete noncompact steady gradient Ricci soliton with positive sectional curvature which satisfies a non-collapsing assumption at infinity. Namely, A three-dimensional complete and noncompact steady gradient Ricci soliton which is nonflat and $\kappa$ noncollapsed, is isometric to the Bryant soliton up to scaling. Under some extra assumptions, it was proved in [1, 6, 7]. A complete proof was recently achieved by Brendle [2] and its generalization can be found in [3].

Another important result is Chen's result [8] saying that any complete noncompact steady gradient Ricci soliton has nonnegative scalar curvature. For certain cases, the lower bounded for the scalar curvature can be improved [10, 12. When 
the scalar curvature of a complete steady gradient Ricci soliton achieves its minimum, Petersen and William 25] proved that such a soliton must be Ricci-flat. On the other hand, if a complete noncompact steady gradient Ricci soliton has positive Ricci curvature and its scalar curvature achieves its maximum, then it must be diffeomorphic to the Euclidean space with the standard metric ([15, 5]); in particular, in this case, such a soliton is Ricci-flat.

To remove the curvature condition, we can prove the following

Proposition 1.1. Suppose $M$ is a compact or complete noncompact manifold of dimension $n$. Then the following conditions are equivalent:

(i) there exists a Ricci-flat Riemannian metric on $M$;

(ii) there exists real numbers $\alpha, \beta$, a smooth function $\phi$ on $M$, and a Riemannian metric $g$ on $M$ such that

$$
0=-R_{i j}+\alpha \nabla_{i} \nabla_{j} \phi, \quad 0=\Delta_{g} \phi+\beta\left|\nabla_{g} \phi\right|_{g}^{2} .
$$

The proof is given in subsection 2.1 .

Remark 1.2. In the compact case, the second condition in 1.2 can be removed. However, in the complete noncompact case, the second condition in 1.2 is necessarily. For example, the cigar soliton is a steady gradient Ricci soliton with nonzero scalar curvature $4 /\left(1+x^{2}+y^{2}\right)$.

The equation 1.2 suggests us to study the parabolic flow

$$
\partial_{t} g(t)=-2 \mathrm{Ric}_{g(t)}+2 \alpha \nabla_{g(t)}^{2} \phi(t), \quad \partial_{t} \phi_{t}=\Delta_{g(t)} \phi(t)+\beta\left|\nabla_{g(t)} \phi(t)\right|_{g(t)}^{2} .
$$

The system (1.3) is similar to the gradient flow of Perelman's entropy functional $\mathcal{W}$ 22. Let $\mathfrak{M e t}(M)$ denote the space of smooth Riemannian metrics on a compact smooth manifold $M$ of dimension $m$. We define Perelman's entropy functional

$$
\mathcal{W}: \mathfrak{M e t}(M) \times C^{\infty}(M) \times \mathbf{R}^{+} \longrightarrow \mathbf{R}
$$

by

$$
\mathcal{W}(g, f, \tau):=\int_{M}\left[\tau\left(R_{g}+\left|\nabla_{g} f\right|_{g}^{2}\right)+f-m\right] \frac{e^{-f}}{(4 \pi \tau)^{m / 2}} d V_{g},
$$

where $d V_{g}$ stands for the volume form of $g$. Perelman's showed that the gradient flow of 1.4$)$ is

$$
\begin{aligned}
\partial_{t} g(t) & =-2 \operatorname{Ric}_{g(t)}-2 \nabla_{g(t)}^{2} f(t), \\
\partial_{t} f(t) & =-\Delta_{g(t)} f(t)-R_{g(t)}+\frac{m}{2 \tau(t)}, \\
\frac{d}{d t} \tau(t) & =-1 ;
\end{aligned}
$$

moreover, the entropy $\mathcal{W}$ is nondecreasing along 1.5 . Since $\mathcal{W}$ is diffeomorphic invariant, i.e.,

$$
\mathcal{W}\left(\Phi^{*} g, \Phi^{*} f, \tau\right)=\mathcal{W}(g, f, \tau)
$$

for any diffeomorphisms $\Phi$ on $M$, it follows that the system 1.5$)$ is equivalent to

$$
\begin{aligned}
\partial_{t} g(t) & =-2 \operatorname{Ric}_{g(t)}, \\
\partial_{t} f(t) & =-\Delta_{g(t)} f(t)+\left|\nabla_{g(t)} f(t)\right|_{g(t)}^{2}-R_{g(t)}+\frac{m}{2 \tau(t)}, \\
\frac{d}{d t} \tau(t) & =-1
\end{aligned}
$$


Thus, (1.3) is a mixture of 1.5$)$ and $(1.6)$. There also are lots of interesting generalized Ricci flows, for example, see [14, 17, 18, 19, 20, 21.

1.3. A parabolic flow. In this paper, we consider a class of Ricc flow type parabolic differential equation:

$$
\begin{aligned}
& \partial_{t} g(t)=-2 \mathrm{Ric}_{g(t)}+2 \alpha_{1} \nabla_{g(t)} \phi(t) \otimes \nabla_{g(t)} \phi(t)+2 \alpha_{2} \nabla_{g(t)}^{2} \phi(t), \\
& \partial_{t} \phi(t)=\Delta_{g(t)} \phi(t)+\beta_{1}\left|\nabla_{g(t)} \phi(t)\right|_{g(t)}^{2}+\beta_{2} \phi(t),
\end{aligned}
$$

where $\alpha_{1}, \alpha_{2}, \beta_{1}, \beta_{2}$ are given constants. When $\alpha_{1}=\alpha_{2}=\beta_{1}=\beta_{2}=\phi(t)=0$, the system (1.7)-1.8) is exactly the Ricci flow introduced by Hamilton [13. When $\alpha_{2}=\beta_{1}=\beta_{2}=0$, it reduces to List's flow [19]. Recently, Hu and Shi 27] introduced a static flow on complete noncompact manifold that is similar to our flow. The main result is

Theorem 1.3. Let $(M, g)$ be an m-dimensional complete and noncompact Riemannian manifold with $\left|\mathrm{Rm}_{g}\right|^{2} \leq k_{0}$ on $M$ and $\phi$ a smooth function on $M$ satisfying $|\phi|^{2}+\left|\nabla_{g} \phi\right|_{g}^{2} \leq k_{1}$ and $\left|\nabla_{g}^{2} \phi\right|_{g}^{2} \leq k_{2}$. Then there exists a positive constant $T$, depending only on $m, k_{0}, k_{1}, k_{2}, \alpha_{1}, \alpha_{2}, \beta_{1}, \beta_{2}$, such that the $\star$-regular $\left(\alpha_{1}, \alpha_{2}, \beta_{1}, \beta_{2}\right)$-flow (1.7)-(1.8) with the initial data $(g, \phi)$ has a smooth solution $(g(t), \phi(t))$ on $M \times[0, T]$ and satisfies the following curvature estimate. For any nonnegative integer $n$, there exist uniform positive constants $C_{k}$, depending only on $m, n, k_{0}, k_{1}, k_{2}, \alpha_{1}, \alpha_{2}, \beta_{1}, \beta_{2}$, such that

on $M \times[0, T]$.

$$
\left|\nabla_{g(t)}^{n} \mathrm{Rm}_{g(t)}\right|_{g(t)}^{2} \leq \frac{C_{n}}{t^{n}}, \quad\left|\nabla_{g(t)}^{n+2} \phi(t)\right|_{g(t)}^{2} \leq \frac{C_{n}}{t^{n}}
$$

For the definition of regular flow and $\star$-regular flow, see Definition 2.11 and Section 3 .

1.4. Notions and convenience. Manifolds are denote by $M, N, \cdots$. If $g$ is a Riemannian metric on $M$, we write $\mathrm{Rm}_{g}, \operatorname{Ric}_{g}, R_{g}, \nabla_{g(t)}$, and $d V_{g}$ the Riemann curvature, Ricci curvature, scalar curvature, Levi-Civita connection, and volume form of $g$, respectively. In our notions, we have

$\Gamma_{i j}^{k}=\frac{1}{2} g^{k \ell}\left(\frac{\partial}{\partial x^{i}} g_{j \ell}+\frac{\partial}{\partial x^{j}} g_{i \ell}-\frac{\partial}{\partial x^{\ell}} g_{i j}\right), R_{i j k}^{\ell}=\frac{\partial}{\partial x^{i}} \Gamma_{j k}^{\ell}-\frac{\partial}{\partial x^{j}} \Gamma_{i k}^{\ell}+\Gamma_{j k}^{p} \Gamma_{i p}^{\ell}-\Gamma_{i k}^{p} \Gamma_{j p}^{\ell}$.

$\nabla^{2} \alpha$ stands for the Hessian of a tensor field $\alpha$. The Lie derivative along a vector field $X$ is denoted by $\mathscr{L}_{X}$.

If we have a family of Riemannian metrics indexed by time $t$, we then write $g(t)$ or $g(x, t)$ for the Riemannian metric at time $t$. The time derivative is denoted by $\partial_{t}$ or $\partial / \partial t$.

For two differential operators $A, B$ on a manifold $M$, define

$$
[A, B]:=A B-B A \text {. }
$$

For example, the Riemann curvature tensor field $\mathrm{Rm}_{g}$ can be written as

$$
\operatorname{Rm}_{g}(X, Y) Z=\left[\nabla_{X}, \nabla_{Y}\right] Z-\nabla_{[X, Y]} Z .
$$

A uniform constant $C$ is a constant depending only on the given data not on the time $t$. Different uniform constants may be labeled by $C_{1}, C_{2}, \cdots$, according to the context. 
If $\mathcal{P}$ and $\mathcal{Q}$ are two quantities (may depend on time) satisfying $\mathcal{P} \leq C \mathcal{Q}$ for some positive uniform constant $C$, then we set

$$
\mathcal{P} \lesssim \mathcal{Q}
$$

Similarly, we can define $\mathcal{P} \approx \mathcal{Q}$ if $\mathcal{P} \lesssim \mathcal{Q}$ and $\mathcal{Q} \lesssim \mathcal{P}$; that is

$$
\frac{1}{C} \mathcal{Q} \leq \mathcal{P} \leq C \mathcal{Q}
$$

for some positive uniform constant $C$.

We usually raise and lower indices for tensor fields; for example,

$$
R_{i j k}{ }^{\ell}=g^{p \ell} R_{i j k p}=R_{i j k}^{\ell}, \quad R_{i}{ }^{\ell}{ }^{\ell}=g^{j p} g^{\ell q} R_{i p k q} .
$$

We also use the Einstein summation for tensor fields; for example,

$$
\langle a, b\rangle_{g}=a_{i j} b^{i j}:=\sum_{1 \leq i, j \leq m} a_{i j} b^{i j}=\sum_{1 \leq i, j, k, \ell \leq m} g^{i k} g^{j \ell} a_{i j} b_{k \ell}
$$

for any any two 2-tensor fields $a=\left(a_{i j}\right)$ and $b=\left(b_{i j}\right)$ on a Riemannian manifold $(M, g)$ of dimension $m$. Moreover we have the Ricci identity

$$
\begin{aligned}
{\left[\nabla_{i}, \nabla_{j}\right] \alpha_{k_{1} \cdots k_{r}}{ }^{\ell_{1} \cdots \ell_{s}}=} & -\sum_{h=1}^{r} R_{i j k_{h}}^{p} \alpha_{k_{1} \cdots k_{h-1} p k_{h+1} \cdots h_{r}}{ }^{\ell_{1} \cdots \ell_{s}} \\
& +\sum_{h=1}^{s} R_{i j p}^{\ell_{h}} \alpha_{k_{1} \cdots k_{r}}{ }^{\ell_{1} \cdots \ell_{h-1} p \ell_{h+1} \cdots \ell_{s}},
\end{aligned}
$$

and the contracted Bianchi identities:

$$
\nabla^{i} R_{i j}=\frac{1}{2} \nabla_{j} R_{g}, \quad \nabla^{\ell} R_{i j k \ell}=\nabla_{i} R_{j k}-\nabla_{j} R_{i k} .
$$

If $A$ and $B$ are two tensor fields on a Riemannian manifold $(M, g)$ we denote by $A * B$ any quantity obtained from $A \otimes B$ by one or more of these operations (a slightly different from that in [9]):

(1) summation over pairs of matching upper and lower indices,

(2) multiplication by constants depending only on the dimension of $M$ and the ranks of $A$ and $B$.

We also denote by $A^{k}$ any $k$-fold product $A \cdots A$. The above product $\langle a, b\rangle_{g}$ can be written as

$$
\langle a, b\rangle_{g}=a * b ;
$$

in order to stress the metric $g$, we also write it as

$$
\langle a, b\rangle_{g}=g^{-1} * g^{-1} * a * b .
$$

\section{A parabolic GeOMEtRic Flow}

In this section we introduce a parabolic geometric flow motivated by 1.2 . At first we will prove Proposition 1.1 
2.1. A characterization of Ricci-flat metrics. Recall that a steady gradient Ricci soliton is a triple $(M, g, f)$ satisfying (1.1).

Proposition 2.1. (See also Proposition 1.1) Suppose $M$ is a compact or complete noncompact manifold of dimension $m$. Then the following conditions are equivalent:

(i) there exists a Ricci-flat Riemannian metric on $M$;

(ii) there exists real numbers $\alpha, \beta$, a smooth function $\phi$ on $M$, and a Riemannian metric $g$ on $M$ such that

$$
0=-R_{i j}+\alpha \nabla_{i} \nabla_{j} \phi, \quad 0=\Delta_{g} \phi+\beta\left|\nabla_{g} \phi\right|_{g}^{2} .
$$

Proof. One direction (i) $\Rightarrow$ (ii) is trivial, since we can take $\alpha=\beta=\phi=0$. In the following we assume that the equation (2.1) holds for some $\alpha, \beta$, and $\phi, g$. When $M$ is compact, a result of Hamilton [15] tells us that $g$ must be Ricci-flat. Now we assume that $M$ is complete noncompact.

Taking the trace of the first equation in 2.1 , we get

$$
R_{g}=\alpha \Delta_{g} \phi
$$

In particular,

$$
R_{g}=-\alpha \beta\left|\nabla_{g} \phi\right|_{g}^{2} .
$$

Hence, if $\alpha \beta \geq 0$, then $R_{g} \leq 0$; on the other hand, by a result of Chen [8], we know that any complete noncompact steady gradient Ricci soliton has nonnegative scalar curvature. Together with those two inequalities, we must have $R_{g}=0$ and $\nabla_{g} \phi=0$ by (2.3). Consequently, from 2.1 , we see that $R_{i j}=0$.

To deal with the case $\alpha \beta<0$, we take the derivative $\nabla^{i}$ on the first equation of 2.1):

$$
0=-\frac{1}{2} \nabla_{j} R_{g}+\alpha \Delta_{g} \nabla_{j} \phi
$$

since $\nabla^{i} R_{i j}=\frac{1}{2} \nabla_{j} R_{g}$. According to the identity $\Delta_{g} \nabla_{j} \phi=\nabla_{j} \Delta_{g} \phi+R_{j k} \nabla^{k} \phi$, we arrive at

$$
0=-\frac{1}{2} \nabla_{j} R_{g}+\alpha\left(\nabla_{j} \Delta_{g} \phi+R_{j k} \nabla^{k} \phi\right) .
$$

Using (2.1) and (2.3), we obtain

$$
\begin{aligned}
0 & =-\frac{1}{2} \nabla_{j} R_{g}+\alpha \nabla_{j}\left(-\beta\left|\nabla_{g} \phi\right|_{g}^{2}\right)+\alpha^{2} \nabla_{j} \nabla_{k} \phi \nabla^{k} \phi \\
& =-\frac{1}{2} \nabla_{j} R_{g}-\alpha \beta \nabla_{j}\left|\nabla_{g} \phi\right|_{g}^{2}+\frac{\alpha^{2}}{2} \nabla_{j}\left|\nabla_{g} \phi\right|_{g}^{2} \\
& =\nabla_{j}\left[\left(\frac{\alpha^{2}}{2}-\alpha \beta\right)\left|\nabla_{g} \phi\right|_{g}^{2}-\frac{1}{2} R_{g}\right]=\frac{\alpha(\alpha-\beta)}{2} \nabla_{j}\left|\nabla_{g} \phi\right|_{g}^{2} .
\end{aligned}
$$

In the case $\alpha \beta<0$, we must have $\alpha \neq 0, \beta \neq 0$, and $\alpha \neq \beta$, so the above identity yields

$$
\left|\nabla_{g} \phi\right|_{g}^{2}=c
$$

for some constant $c$, and hence $R_{g}=-\alpha \beta c$ using again (2.1). From the proved identity $0=-\frac{1}{2} \nabla_{j} R_{g}+\alpha \Delta_{g} \nabla_{j} \phi$, we obtain $\Delta_{g} \nabla_{j} \phi=0$. Consequently

$$
0=2 \nabla^{j} \phi \Delta_{g} \nabla_{j} \phi=\Delta_{g}\left|\nabla_{g} \phi\right|_{g}^{2}-2\left|\nabla_{g}^{2} \phi\right|_{g}^{2}=0-2\left|\nabla_{g}^{2} \phi\right|_{g}^{2}
$$

and then $\left|\nabla_{g}^{2} \phi\right|_{g}^{2}=0$. In particular, $\nabla_{i} \nabla_{j} \phi=0$ and hence $R_{i j}=0$. In each case, we get a Ricci-flat metric. 
2.2. Evolution equations. Motivated by Proposition 2.1. we consider a class of Ricc flow type parabolic differential equation:

$$
\begin{aligned}
& \partial_{t} g(t)=-2 \operatorname{Ric}_{g(t)}+2 \alpha_{1} \nabla_{g(t)} \phi(t) \otimes \nabla_{g(t)} \phi(t)+2 \alpha_{2} \nabla_{g(t)}^{2} \phi(t), \\
& \partial_{t} \phi(t)=\Delta_{g(t)} \phi(t)+\beta_{1}\left|\nabla_{g(t)} \phi(t)\right|_{g(t)}^{2}+\beta_{2} \phi(t),
\end{aligned}
$$

where $\alpha_{1}, \alpha_{2}, \beta_{1}, \beta_{2}$ are given constants. When $\alpha_{1}=\alpha_{2}=\beta_{1}=\beta_{2}=\phi(t)=0$, the system 1.7 -1.8 is exactly the Ricci flow introduced by Hamilton [13]. When $\alpha_{2}=\beta_{1}=\beta_{2}=0$, it reduces to List's flow [19].

To compute evolution equations for $2.4-2.5$, we recall variation formulas stated in [9]. Consider a flow

$$
\partial_{t} g(t)=h(t)
$$

where $h(t)$ is a family of symmetric 2 -tensor fields. Then

$$
\begin{aligned}
\partial_{t} g^{i j}= & -g^{i k} g^{j \ell} h_{k \ell}, \\
\partial_{t} \Gamma_{i j}^{k}= & \frac{1}{2} g^{k \ell}\left(\nabla_{i} h_{j \ell}+\nabla_{j} h_{i \ell}-\nabla_{\ell} h_{i j}\right), \\
\partial_{t} R_{i j k}^{\ell}= & \frac{1}{2} g^{\ell p}\left(\nabla_{i} \nabla_{j} h_{k p}+\nabla_{i} \nabla_{k} h_{j p}-\nabla_{i} \nabla_{p} h_{j k}\right. \\
& \left.-\nabla_{j} \nabla_{i} h_{k p}-\nabla_{j} \nabla_{k} h_{i p}+\nabla_{j} \nabla_{p} h_{i k}\right), \\
\partial_{t} R_{j k}= & \frac{1}{2} g^{p q}\left(\nabla_{q} \nabla_{j} h_{k p}+\nabla_{q} \nabla_{k} h_{j p}-\nabla_{q} \nabla_{p} h_{j k}-\nabla_{j} \nabla_{k} h_{q p}\right), \\
\partial_{t} R_{g(t)}= & -\Delta_{g(t)} \operatorname{tr}_{g(t)} h(t)+\operatorname{div}_{g(t)}\left(\operatorname{div}_{g(t)} h(t)\right)-\left\langle h(t), \operatorname{Ric}_{g(t)}\right\rangle_{g(t)}, \\
\partial_{t} d V_{g(t)}= & \frac{1}{2} \operatorname{tr}_{g(t)} h(t) d V_{g(t)} .
\end{aligned}
$$

We now take

$$
h(t):=-2 \operatorname{Ric}_{g(t)}+2 \alpha_{1} d \phi(t) \otimes d \phi(t)+2 \alpha_{2} \nabla_{g(t)}^{2} \phi(t) .
$$

Lemma 2.2. Under 2.4 2.5, we have

$$
\begin{aligned}
\partial_{t} \Gamma_{i j}^{k}= & -\nabla_{i} R_{j}{ }^{k}-\nabla_{j} R_{i}{ }^{k}+\nabla^{k} R_{i j}+2 \alpha_{1} \nabla_{i} \nabla_{j} \phi(t) \cdot \nabla^{k} \phi(t) \\
& +\alpha_{2} \nabla^{k} \nabla_{i} \nabla_{j} \phi(t)-\alpha_{2}\left(R_{i}{ }^{k}{ }_{j}{ }^{p} \nabla_{p} \phi(t)+R_{j}{ }^{k}{ }_{i}{ }^{p} \nabla_{p} \phi(t)\right) .
\end{aligned}
$$

Proof. Compute

$$
\begin{aligned}
\partial_{t} \Gamma_{i j}^{k}= & -\nabla_{i} R_{j}{ }^{k}-\nabla_{j} R_{i}{ }^{k}+\nabla^{k} R_{i j}+2 \alpha_{1} \nabla_{i} \nabla_{j} \phi(t) \cdot \nabla^{k} \phi(t) \\
& +\alpha_{2} g^{k \ell}\left(\nabla_{i} \nabla_{j} \nabla_{\ell} \phi(t)+\nabla_{j} \nabla_{i} \nabla_{\ell} \phi(t)-\nabla_{\ell} \nabla_{i} \nabla_{j} \phi(t)\right) .
\end{aligned}
$$

According to the Ricci identity, we have

$$
\begin{aligned}
& \nabla_{i} \nabla_{j} \nabla_{\ell} \phi(t)=\nabla_{i} \nabla_{\ell} \nabla_{j} \phi(t)=\nabla_{\ell} \nabla_{i} \nabla_{j} \phi(t)-R_{i \ell j}^{p} \nabla_{p} \phi(t), \\
& \nabla_{j} \nabla_{i} \nabla_{\ell} \phi(t)=\nabla_{j} \nabla_{\ell} \nabla_{i} \phi(t)=\nabla_{\ell} \nabla_{j} \nabla_{i} \phi(t)-R_{j \ell i}^{p} \nabla_{p} \phi(t) ;
\end{aligned}
$$

thus we prove the desired result.

Lemma 2.3. Under 2.4 2.5), we have

$$
\begin{aligned}
\partial_{t} R_{i j}= & \Delta_{g(t)} R_{i j}-2 R_{i k} R_{j}^{k}+2 R_{p i j q} R^{p q}-2 \alpha_{1} R_{p i j q} \nabla^{p} \phi(t) \nabla^{q} \phi(t) \\
& +2 \alpha_{1} \Delta_{g(t)} \phi(t) \cdot \nabla_{i} \nabla_{j} \phi(t)-2 \alpha_{1} \nabla_{i} \nabla_{k} \phi(t) \nabla^{k} \nabla_{j} \phi(t) \\
& +\alpha_{2}\left(R_{i}{ }^{p} \nabla_{p} \nabla_{j} \phi(t)+R_{j}{ }^{p} \nabla_{p} \nabla_{i} \phi(t)+\nabla_{p} R_{i j} \nabla^{p} \phi(t)\right) .
\end{aligned}
$$


Proof. Note that

$$
\partial_{t} R_{i j}=-\frac{1}{2} \Delta_{g(t)} h_{i j}-\frac{1}{2} \nabla_{i} \nabla_{j}\left(g^{p q} h_{p q}\right)+\frac{1}{2} g^{p q}\left(\nabla_{p} \nabla_{j} h_{i q}+\nabla_{p} \nabla_{i} h_{j q}\right) .
$$

Denote by $I_{i}, i=1,2,3,4$, the $i$ th term on the right-hand side of the above equation. For $I_{1}$ we have

$$
\begin{aligned}
I_{1}= & -\frac{1}{2} \Delta_{g(t)}\left[-2 R_{i j}+2 \alpha_{1} \nabla_{i} \phi(t) \nabla_{j} \phi(t)+2 \alpha_{2} \nabla_{i} \nabla_{j} \phi(t)\right] \\
= & \Delta_{g(t)} R_{i j}-\alpha_{1} \Delta_{g(t)} \nabla_{i} \phi(t) \nabla_{j} \phi(t)-\alpha_{1} \nabla_{i} \phi(t) \Delta_{g(t)} \nabla_{j} \phi(t) \\
& -2 \alpha_{1} \nabla_{k} \nabla_{i} \phi(t) \nabla^{k} \nabla_{j} \phi(t)-\alpha_{2} \Delta_{g(t)}\left(\nabla_{i} \nabla_{j} \phi(t)\right) .
\end{aligned}
$$

Since $\left|\nabla_{g(t)} \phi\right|_{g(t)}^{2}$ is a function, it follows $\nabla_{i} \nabla_{j}\left|\nabla_{g(t)} \phi(t)\right|_{g(t)}^{2}=\nabla_{j} \nabla_{i}\left|\nabla_{g(t)} \phi(t)\right|_{g(t)}^{2}$. Hence

$$
\begin{aligned}
I_{2}= & -\frac{1}{2} \nabla_{i} \nabla_{j}\left(-2 R_{g(t)}+2 \alpha_{1}\left|\nabla_{g(t)} \phi(t)\right|_{g(t)}^{2}+2 \alpha_{2} \Delta_{g(t)} \phi(t)\right) \\
= & \nabla_{i} \nabla_{j} R_{g(t)}-\frac{1}{2} \alpha_{1} \nabla_{i} \nabla_{j}\left|\nabla_{g(t)} \phi(t)\right|_{g(t)}^{2}-\frac{1}{2} \alpha_{1} \nabla_{j} \nabla_{i}\left|\nabla_{g(t)} \phi(t)\right|_{g(t)}^{2} \\
& -\alpha_{2} \nabla_{i} \nabla_{j}\left(\Delta_{g(t)} \phi(t)\right) \\
= & \nabla_{i} \nabla_{j} R_{g(t)}-\alpha_{1}\left(\nabla_{i} \nabla_{j} \nabla_{k} \phi(t)+\nabla_{j} \nabla_{i} \nabla_{k} \phi(t)\right) \nabla^{k} \phi(t) \\
& -2 \alpha_{1} \nabla_{i} \nabla_{k} \phi(t) \nabla_{j} \nabla^{k} \phi(t)-\alpha_{2} \nabla_{i} \nabla_{j}\left(\Delta_{g(t)} \phi(t)\right) .
\end{aligned}
$$

The symmetry of $I_{3}$ and $I_{4}$ allows us to consider only one term, saying for example $I_{3}$. Then

$$
\begin{aligned}
I_{3}= & \frac{1}{2} g^{p q} \nabla_{p} \nabla_{j}\left(-2 R_{i q}+2 \alpha_{1} \nabla_{i} \phi(t) \nabla_{q} \phi(t)+2 \alpha_{2} \nabla_{i} \nabla_{q} \phi(t)\right) \\
= & -g^{p q}\left(\nabla_{j} \nabla_{p} R_{i q}-R_{p j i}^{k} R_{k q}-R_{p j q}^{k} R_{i k}\right)+\alpha_{1} g^{p q}\left[\nabla_{p} \nabla_{j} \nabla_{i} \phi(t) \nabla_{q} \phi(t)\right. \\
& \left.+\nabla_{j} \nabla_{i} \phi(t) \nabla_{p} \nabla_{q} \phi(t)+\nabla_{p} \nabla_{i} \phi(t) \nabla_{j} \nabla_{q} \phi(t)+\nabla_{i} \phi(t) \nabla_{p} \nabla_{j} \nabla_{q} \phi(t)\right] \\
& +\alpha_{2} \nabla^{q} \nabla_{j}\left(\nabla_{i} \nabla_{q} \phi(t)\right) ;
\end{aligned}
$$

since

$$
\nabla_{p} \nabla_{j} \nabla_{i} \phi(t)=\nabla_{p} \nabla_{i} \nabla_{j} \phi(t)=\nabla_{i} \nabla_{p} \nabla_{j} \phi(t)-R_{p i j}^{k} \nabla_{k} \phi(t),
$$

it follows that

$$
\begin{aligned}
I_{3}= & -\frac{1}{2} \nabla_{i} \nabla_{j} R_{g(t)}+R_{p i j q} R^{p q}-R_{i k} R^{k}{ }_{j}+\alpha_{1}\left[\nabla_{i} \nabla_{j} \nabla_{p} \phi(t) \nabla^{p} \phi(t)\right. \\
& \left.-R_{p i j q} \nabla^{p} \phi(t) \nabla^{q} \phi(t)\right]+\alpha_{1}\left[\Delta_{g(t)} \phi(t) \nabla_{i} \nabla_{j} \phi(t)+\nabla_{i} \nabla_{p} \phi(t) \nabla_{j} \nabla^{p} \phi(t)\right. \\
& \left.+\nabla_{i} \phi(t) \Delta_{g(t)} \nabla_{j} \phi(t)\right]+\alpha_{2} \nabla^{q} \nabla_{j}\left(\nabla_{i} \nabla_{q} \phi(t)\right) .
\end{aligned}
$$

Consequently, we arrive at

$$
\begin{aligned}
\partial_{t} R_{i j}= & \Delta_{g(t)} R_{i j}-2 R_{i k} R^{k}{ }_{j}+2 R_{p i j q} R^{p q}-2 \alpha_{1} R_{p i j q} \nabla^{p} \phi(t) \nabla^{q} \phi(t) \\
& +2 \alpha_{1} \Delta_{g(t)} \phi(t) \cdot \nabla_{i} \nabla_{j} \phi(t)-2 \alpha_{1} \nabla_{i} \nabla_{k} \phi(t) \nabla^{k} \nabla_{j} \phi(t)+\Lambda,
\end{aligned}
$$

where

$$
\begin{aligned}
\Lambda:= & -\alpha_{2} \Delta_{g(t)}\left(\nabla_{i} \nabla_{j} \phi(t)\right)-\alpha_{2} \nabla_{i} \nabla_{j}\left(\Delta_{g(t)} \phi(t)\right) \\
& +\alpha_{2} \nabla^{q} \nabla_{j}\left(\nabla_{i} \nabla_{q} \phi(t)\right)+\alpha_{2} \nabla^{q} \nabla_{i}\left(\nabla_{j} \nabla_{q} \phi(t)\right) .
\end{aligned}
$$


This term can be simplified as

$$
\begin{aligned}
\Lambda= & \alpha_{2}\left[\nabla^{q} \nabla_{j}\left(\nabla_{q} \nabla_{i} \phi(t)\right)+\nabla^{q} \nabla_{i}\left(\nabla_{q} \nabla_{j} \phi(t)\right)\right. \\
& \left.-\Delta_{g(t)}\left(\nabla_{i} \nabla_{j} \phi(t)\right)-\nabla_{i} \nabla_{j}\left(\Delta_{g(t)} \phi(t)\right)\right] \\
= & \alpha_{2}\left[\nabla^{q}\left(\nabla_{q} \nabla_{j} \nabla_{i} \phi(t)-R_{j q i}^{p} \nabla_{p} \phi(t)\right)+\nabla^{q}\left(\nabla_{q} \nabla_{i} \nabla_{j} \phi(t)-R_{i q j}^{p} \nabla_{p} \phi(t)\right)\right. \\
& \left.-\Delta_{g(t)}\left(\nabla_{i} \nabla_{j} \phi(t)\right)-\nabla_{i} \nabla_{j}\left(\Delta_{g(t)} \phi(t)\right)\right] \\
= & \alpha_{2}\left[\Delta_{g(t)}\left(\nabla_{i} \nabla_{j} \phi(t)\right)-\nabla_{i} \nabla_{j}\left(\Delta_{g(t)} \phi(t)\right)-2 R_{i p j q} \nabla^{p} \nabla^{q} \phi(t)\right. \\
& \left.-\nabla^{q} R_{j q i p} \cdot \nabla^{p} \phi(t)-\nabla^{q} R_{i q j p} \cdot \nabla^{p} \phi(t)\right] \\
= & \alpha_{2}\left[\Delta_{g(t)}\left(\nabla_{i} \nabla_{j} \phi(t)\right)-\nabla_{i} \nabla_{j}\left(\Delta_{g(t)} \phi(t)\right)-2 R_{i p j q} \nabla^{p} \nabla^{q} \phi(t)\right. \\
& \left.-\nabla_{i} R_{j p} \cdot \nabla^{p} \phi(t)-\nabla_{j} R_{i p} \cdot \nabla^{p} \phi(t)+2 \nabla_{p} R_{i j} \cdot \nabla^{p} \phi(t)\right]
\end{aligned}
$$

where we used the contract Bianchi identity $\nabla^{p} R_{j k \ell p}=\nabla_{j} R_{k \ell}-\nabla_{k} R_{j \ell}$ in the last line. The final step is to simplify the difference $\left[\Delta_{g(t)}, \nabla_{i} \nabla_{j}\right] \phi(t)$. According to the Ricci identity, we have

$$
\begin{aligned}
{\left[\Delta_{g(t)}, \nabla_{i} \nabla_{j}\right] \phi(t)=} & \nabla^{k} \nabla_{k} \nabla_{i} \nabla_{j} \phi(t)=\nabla^{k}\left(\nabla_{i} \nabla_{k} \nabla_{j} \phi(t)-R_{k i j}^{\ell} \nabla_{\ell} \phi(t)\right) \\
= & \nabla_{k} \nabla_{i} \nabla^{k} \nabla_{j} \phi(t)-\nabla^{k} R_{k i j}^{\ell} \nabla_{\ell} \phi(t)-R_{k i j}^{\ell} \nabla^{k} \nabla_{\ell} \phi(t) \\
= & \nabla_{i} \nabla_{k} \nabla_{j} \nabla^{k} \phi(t)-R_{k i j}^{\ell} \nabla_{\ell} \nabla^{k} \phi(t)+R_{k i \ell}^{k} \nabla_{j} \nabla^{\ell} \phi(t) \\
& -\nabla^{k} R_{\ell j i k} \nabla^{\ell} \phi(t)-R_{k i j \ell} \nabla^{k} \nabla^{\ell} \phi(t) \\
= & \nabla_{i} \nabla_{j}\left(\Delta_{g(t)} \phi(t)\right)+\nabla_{i} R_{j \ell} \cdot \nabla^{\ell} \phi(t)+\nabla_{j} R_{i \ell} \nabla^{\ell} \phi(t) \\
& +R_{j \ell} \nabla_{i} \nabla_{\ell} \phi(t)+R_{i \ell} \nabla_{j} \nabla^{\ell} \phi(t) \\
& -2 R_{k i j \ell} \nabla^{k} \nabla^{\ell} \phi(t)-\nabla_{\ell} R_{i j} \nabla^{\ell} \phi(t) .
\end{aligned}
$$

Consequently, $\Lambda=\alpha_{2}\left[R_{i \ell} \nabla^{\ell} \nabla_{j} \phi(t)+R_{j \ell} \nabla^{\ell} \nabla_{i} \phi(t)+\nabla_{\ell} R_{i j} \nabla^{\ell} \phi(t)\right]$.

Lemma 2.4. Under 2.4 (2.5), we have

$$
\begin{aligned}
\partial_{t} R_{g(t)}= & \Delta_{g(t)} R_{g(t)}+2\left|\operatorname{Ric}_{g(t)}\right|_{g(t)}^{2}+2 \alpha_{1}\left|\Delta_{g(t)} \phi(t)\right|_{g(t)}^{2}-2 \alpha_{1}\left|\nabla_{g(t)}^{2} \phi(t)\right|_{g(t)}^{2} \\
& -4 \alpha_{1}\left\langle\operatorname{Ric}_{g(t)}, \nabla_{g(t)} \phi(t) \otimes \nabla_{g(t)} \phi(t)\right\rangle_{g(t)} \\
& +\alpha_{2}\left\langle\nabla_{g(t)} R_{g(t)}, \nabla_{g(t)} \phi(t)\right\rangle_{g(t)} .
\end{aligned}
$$

Proof. By the above formula for $\partial_{t} R_{g(t)}$, we obtain

$$
\begin{aligned}
\partial_{t} R_{g(t)}= & \Delta_{g(t)} R_{g(t)}+2\left|\operatorname{Ric}_{g(t)}\right|_{g(t)}^{2}+2 \alpha_{1}\left|\Delta_{g(t)} \phi(t)\right|_{g(t)}^{2}-2 \alpha_{1}\left|\nabla_{g(t)}^{2} \phi(t)\right|_{g(t)}^{2} \\
& -2 \alpha_{1} R^{i j} \nabla_{i} \phi(t) \nabla_{j} \phi(t)-2 \alpha_{1}\left(\Delta_{g(t)} \nabla_{i} \phi(t)-\nabla_{i} \Delta_{g(t)} \phi(t)\right) \nabla^{i} \phi(t)+I
\end{aligned}
$$

where

$$
I:=-2 \alpha_{2} \Delta_{g(t)}\left(\Delta_{g(t)} \phi(t)\right)+2 \alpha_{2} \nabla^{i} \nabla^{j}\left(\nabla_{i} \nabla_{j} \phi(t)\right)-2 \alpha_{2} R^{i j} \nabla_{i} \nabla_{j} \phi(t) .
$$


By the Ricci identity we have

$$
\begin{aligned}
\Delta_{g(t)} \nabla_{i} \phi(t) & =\nabla^{j} \nabla_{j} \nabla_{i} \phi(t)=\nabla^{j} \nabla_{i} \nabla_{j} \phi(t)=\nabla_{j} \nabla_{i} \nabla^{j} \phi(t) \\
& =\nabla_{i} \Delta_{g(t)} \phi(t)+R_{j i k}^{j} \nabla^{k} \phi(t)=\nabla_{i} \Delta_{g(t)} \phi(t)+R_{i k} \nabla^{k} \phi(t),
\end{aligned}
$$

and

$$
\begin{aligned}
\nabla^{i} \nabla_{j}\left(\nabla_{i} \nabla_{j} \phi(t)\right) & =\nabla^{i}\left(\Delta_{g(t)} \nabla_{i} \phi(t)\right)=\nabla^{i}\left(\nabla_{i} \Delta_{g(t)} \phi(t)+R_{i k} \nabla^{k} \phi(t)\right) \\
& =\Delta_{g(t)}\left(\Delta_{g(t)} \phi(t)\right)+\frac{1}{2} \nabla_{k} R_{g(t)} \nabla^{k} \phi(t)+R_{i k} \nabla^{i} \nabla^{k} \phi(t) .
\end{aligned}
$$

Consequently, we get

$$
I=\alpha_{2}\left\langle\nabla_{g(t)} R_{g(t)}, \nabla_{g(t)} \phi(t)\right\rangle_{g(t)}
$$

and then the desired formula.

Following Hamilton, we introduce the tensor field

$$
B_{i j k \ell}:=-g^{p r} g^{q s} R_{i p j q} R_{k r \ell s} .
$$

Note that $B_{j i \ell k}=B_{i j k \ell}$ and $B_{i j k \ell}=B_{k \ell i j}$.

Lemma 2.5. Under 2.4 2.5), we have

$$
\begin{aligned}
\partial_{t} R_{i j k \ell}= & \Delta_{g(t)} R_{i j k \ell}+2\left(B_{i j k \ell}-B_{i j \ell k}+B_{i k j \ell}-B_{i \ell j k}\right) \\
& -\left(R_{i}{ }^{p} R_{p j k \ell}+R_{j}{ }^{p} R_{i p k \ell}+R_{k}{ }^{p} R_{i j p \ell}+R_{\ell} R_{i j k p}\right) \\
& +2 \alpha_{1}\left[\nabla_{i} \nabla_{\ell} \phi(t) \nabla_{j} \nabla_{k} \phi(t)-\nabla_{i} \nabla_{k} \phi(t) \nabla_{j} \nabla_{\ell} \phi(t)\right] \\
& +\alpha_{2}\left[\nabla^{p} R_{i j k \ell} \nabla_{p} \phi(t)-R_{i j k}{ }^{p} \nabla_{p} \nabla_{\ell} \phi(t)+R^{p}{ }_{j k \ell} \nabla_{i} \nabla_{p} \phi(t)\right. \\
& \left.+R_{i}{ }^{p}{ }_{k \ell} \nabla_{j} \nabla_{p} \phi(t)+R_{i j}{ }^{p} \nabla_{k} \nabla_{p} \phi(t)\right] .
\end{aligned}
$$

Proof. Recall the evolution equation

$\partial_{t} R_{i j k}^{\ell}=\frac{1}{2} g^{\ell p}\left(\nabla_{i} \nabla_{k} h_{j p}+\nabla_{j} \nabla_{p} h_{i k}-\nabla_{i} \nabla_{p} h_{j k}-\nabla_{j} \nabla_{k} h_{i p}-R_{i j k}^{q} h_{q p}-R_{i j p}^{q} h_{k q}\right)$ where $\partial_{t} g_{i j}=h_{i j}$. Applying the above formula to $h_{i j}=-2 R_{i j}+2 \alpha_{1} \nabla_{i} \phi(t) \nabla_{j} \phi(t)+$ $2 \alpha_{2} \nabla_{i} \nabla_{j} \phi(t)$ implies

$$
\begin{aligned}
\partial_{t} R_{i j k}^{\ell}= & g^{\ell p}\left(\nabla_{i} \nabla_{p} R_{j k}+\nabla_{j} \nabla_{k} R_{i p}-\nabla_{i} \nabla_{k} R_{j p}-\nabla_{j} \nabla_{p} R_{i k}\right)+g^{\ell p} \\
& {\left[\alpha_{1} \nabla_{i}\left(\nabla_{k} \nabla_{j} \phi(t) \nabla_{p} \phi(t)+\nabla_{j} \phi(t) \nabla_{k} \nabla_{p} \phi(t)\right)+\alpha_{2} \nabla_{i} \nabla_{k} \nabla_{j} \nabla_{p} \phi(t)\right.} \\
& +\alpha_{1} \nabla_{j}\left(\nabla_{p} \nabla_{i} \phi(t) \nabla_{k} \phi(t)+\nabla_{i} \phi(t) \nabla_{p} \nabla_{k} \phi(t)\right)+\alpha_{2} \nabla_{j} \nabla_{p} \nabla_{i} \nabla_{k} \phi(t) \\
& -\alpha_{1} \nabla_{i}\left(\nabla_{p} \nabla_{j} \phi(t) \nabla_{k} \phi(t)+\nabla_{j} \phi(t) \nabla_{p} \nabla_{k} \phi(t)\right)-\alpha_{2} \nabla_{i} \nabla_{p} \nabla_{j} \nabla_{k} \phi(t) \\
& \left.-\alpha_{1} \nabla_{j}\left(\nabla_{k} \nabla_{i} \phi(t) \nabla_{p} \phi(t)+\nabla_{i} \phi(t) \nabla_{k} \nabla_{p} \phi(t)\right)-\alpha_{2} \nabla_{j} \nabla_{k} \nabla_{i} \nabla_{p} \phi(t)\right] \\
& +g^{\ell p}\left[R_{i j k}^{q}\left(R_{q p}-\alpha_{1} \nabla_{q} \phi(t) \nabla_{p} \phi(t)-\alpha_{2} \nabla_{q} \nabla_{p} \phi(t)\right)\right. \\
& \left.+R_{i j p}^{q}\left(R_{k q}-\alpha_{1} \nabla_{k} \phi(t) \nabla_{q} \phi(t)-\alpha_{2} \nabla_{k} \nabla_{q} \phi(t)\right)\right]:=I_{1}+I_{2}+I_{3},
\end{aligned}
$$


where

$$
\begin{aligned}
I_{1}:= & g^{\ell p}\left(\nabla_{i} \nabla_{p} R_{j k}+\nabla_{j} \nabla_{k} R_{i p}-\nabla_{i} \nabla_{k} R_{j p}-\nabla_{j} \nabla_{p} R_{i k}+R_{i j k}^{q} R_{q p}+R_{i j p}^{q} R_{k q}\right), \\
I_{3}:= & g^{\ell p}\left[R_{i j k}^{q}\left(-\alpha_{1} \nabla_{q} \phi(t) \nabla_{p} \phi(t)-\alpha_{2} \nabla_{q} \nabla_{p} \phi(t)\right)\right. \\
& \left.+R_{i j p}^{q}\left(-\alpha_{1} \nabla_{k} \phi(t) \nabla_{q} \phi(t)-\alpha_{2} \nabla_{k} \nabla_{q} \phi(t)\right)\right] \\
= & -\left(\alpha_{1} R_{i j k}^{q} \nabla_{q} \phi(t) \nabla^{\ell} \phi(t)+\alpha_{2} R_{i j k}^{q} \nabla_{q} \nabla^{\ell} \phi(t)\right) \\
& -\left(\alpha_{1} R_{i j}{ }^{\ell q} \nabla_{k} \phi(t) \nabla_{q} \phi(t)+\alpha_{2} R_{i j}{ }^{\ell q} \nabla_{k} \nabla_{q} \phi(t)\right), \\
I_{2}:= & \text { the rest terms. }
\end{aligned}
$$

According to 9, 13] we have

$$
\begin{aligned}
I_{1}= & \Delta_{g(t)} R_{i j k}^{\ell}+g^{p q}\left(R_{i j p}^{r} R_{r q k}^{\ell}-2 R_{p i k}^{r} R_{j q r}^{\ell}+2 R_{p i r}^{\ell} R_{j q k}^{r}\right) \\
& -R_{i}{ }^{r} R_{r j k}^{\ell}-R_{j}{ }^{r} R_{i r k}^{\ell}-R_{k}{ }^{r} R_{i j r}^{\ell}+R_{r}{ }^{\ell} R_{i j k}^{r} .
\end{aligned}
$$

It can be showed that

$$
\begin{aligned}
I_{2}= & g^{\ell p}\left[\alpha _ { 1 } \left(\nabla_{i} \nabla_{j} \nabla_{k} \phi(t) \nabla_{p} \phi(t)+\nabla_{j} \nabla_{i} \nabla_{p} \phi(t) \nabla_{k} \phi(t)\right.\right. \\
& \left.-\nabla_{i} \nabla_{j} \nabla_{p} \phi(t) \nabla_{k} \phi(t)-\nabla_{j} \nabla_{i} \nabla_{k} \phi(t) \nabla_{p} \phi(t)\right)+2 \alpha_{1}\left(\nabla_{k} \nabla_{j} \phi(t) \nabla_{i} \nabla_{p} \phi(t)\right. \\
& \left.\left.-\nabla_{k} \nabla_{i} \phi(t) \nabla_{j} \nabla_{p} \phi(t)\right)\right]+\alpha_{2}\left(\nabla_{i} \nabla_{k} \nabla_{j} \nabla_{p} \phi(t)+\nabla_{j} \nabla_{p} \nabla_{i} \nabla_{k} \phi(t)\right. \\
& \left.-\nabla_{j} \nabla_{k} \nabla_{i} \nabla_{p} \phi(t)-\nabla_{i} \nabla_{p} \nabla_{j} \nabla_{k} \phi(t)\right) \\
= & -\alpha_{1} R_{i j k}^{q} \nabla_{q} \phi(t) \nabla^{\ell} \phi(t)+\alpha_{1} R_{i j}{ }^{\ell q} \nabla_{q} \phi(t) \nabla_{k} \phi(t)+2 \alpha_{1}\left(\nabla_{i} \nabla^{\ell} \phi(t) \nabla_{k} \nabla_{j} \phi(t)\right. \\
& \left.-\nabla_{i} \nabla_{k} \phi(t) \nabla_{j} \nabla^{\ell} \phi(t)\right)+\alpha_{2}\left(\nabla_{i} \nabla_{k} \nabla_{j} \nabla^{\ell} \phi(t)+\nabla_{j} \nabla^{\ell} \nabla_{i} \nabla_{k} \phi(t)\right. \\
& \left.-\nabla_{j} \nabla_{k} \nabla_{i} \nabla^{\ell} \phi(t)-\nabla_{i} \nabla^{\ell} \nabla_{j} \nabla_{k} \phi(t)\right)
\end{aligned}
$$

together with $I_{3}$, we arrive at

$$
\begin{aligned}
I_{2}+I_{3}= & -2 \alpha_{1} R_{i j k}^{q} \nabla_{q} \phi(t) \nabla^{\ell} \phi(t)-\alpha_{2}\left(R_{i j k}^{q} \nabla_{q} \nabla^{\ell} \phi(t)+R_{i j}^{\ell q} \nabla_{k} \nabla_{q} \phi(t)\right) \\
& +2 \alpha_{1}\left(\nabla_{i} \nabla^{\ell} \phi(t) \nabla_{k} \nabla_{j} \phi(t)-\nabla_{i} \nabla_{k} \phi(t) \nabla_{j} \nabla^{\ell} \phi(t)\right) \\
& +\alpha_{2}\left(\nabla_{i} \nabla_{k} \nabla_{j} \nabla^{\ell} \phi(t)+\nabla_{j} \nabla^{\ell} \nabla_{i} \nabla_{k} \phi(t)\right. \\
& \left.-\nabla_{j} \nabla_{k} \nabla_{i} \nabla^{\ell} \phi(t)-\nabla_{i} \nabla^{\ell} \nabla_{j} \nabla_{k} \phi(t)\right)
\end{aligned}
$$


Using the Ricci identity, the last term on the right-hand side of $I_{2}+I_{3}$ can be simplified as

$$
\begin{aligned}
\nabla_{i} \nabla_{k} \nabla_{j} \nabla^{\ell} \phi(t)-\nabla_{i} \nabla^{\ell} \nabla_{j} \nabla_{k} \phi(t) & =g^{\ell p}\left(\nabla_{i} \nabla_{k} \nabla_{j} \nabla_{p} \phi(t)-\nabla_{i} \nabla_{p} \nabla_{j} \nabla_{k} \phi(t)\right) \\
& =g^{\ell p} \nabla_{i}\left(\nabla_{k} \nabla_{p} \nabla_{j} \phi(t)-\nabla_{p} \nabla_{k} \nabla_{j} \phi(t)\right) \\
& =-g^{\ell p} \nabla_{i}\left(R_{k p j}^{q} \nabla_{q} \phi(t)\right) \\
& =-\nabla_{i} R_{k}{ }^{\ell}{ }^{q} \nabla_{q} \phi(t)-R_{k}{ }^{\ell}{ }^{q} \nabla_{i} \nabla_{q} \phi(t), \\
\nabla_{j} \nabla^{\ell} \nabla_{i} \nabla_{k} \phi(t)-\nabla_{j} \nabla_{k} \nabla_{i} \nabla^{\ell} \phi(t) & =g^{\ell p} \nabla_{j}\left(\nabla_{p} \nabla_{i} \nabla_{k} \phi(t)-\nabla_{k} \nabla_{i} \nabla_{p} \phi(t)\right) \\
& =g^{\ell p} \nabla_{j}\left(-R_{p k i}^{q} \nabla_{q} \phi(t)\right) \\
& =-\nabla_{j} R^{\ell}{ }_{k i}{ }^{q} \nabla_{q} \phi(t)-R^{\ell}{ }_{k i}{ }^{q} \nabla_{j} \nabla_{q} \phi(t) .
\end{aligned}
$$

Consequently,

$$
\begin{aligned}
I_{2}+I_{3}= & -2 \alpha_{1} R_{i j k}^{q} \nabla_{q} \phi(t) \nabla^{\ell} \phi(t) \\
& +2 \alpha_{1}\left(\nabla_{i} \nabla^{\ell} \phi(t) \nabla_{k} \nabla_{j} \phi(t)-\nabla_{i} \nabla_{k} \phi(t) \nabla_{j} \nabla^{\ell} \phi(t)\right) \\
& +\alpha_{2}\left(\nabla^{q} R_{i j k}{ }^{\ell}-R_{k}{ }^{\ell}{ }^{q}{ }^{q} \nabla_{i} \nabla_{q} \phi(t)-R^{\ell}{ }_{k i}{ }^{q} \nabla_{j} \nabla_{q} \phi(t)\right. \\
& \left.-R_{i j k}{ }^{q} \nabla_{q} \nabla^{\ell} \phi(t)-R_{i j}{ }^{\ell q} \nabla_{k} \nabla_{q} \phi(t)\right),
\end{aligned}
$$

because

$\nabla_{i} R_{k}{ }^{\ell}{ }^{q}+\nabla_{j} R_{k i}^{\ell}{ }^{q}=g^{\ell s} g^{p q}\left(\nabla_{i} R_{j p k s}+\nabla_{j} R_{p i k s}\right)=-g^{\ell s} g^{q p} \nabla_{p} R_{i j k s}=-\nabla^{q} R_{i j k}{ }^{\ell}$.

Replacing $\ell$ by $s$ in $I_{1}, I_{2}, I_{3}$, we have

$$
\partial_{t} R_{i j k}^{s}=\tilde{I}_{1}+\tilde{I}_{2}+\tilde{I}_{3}
$$

where we denote by $\tilde{I}_{i}$ the corresponding terms; hence

$$
\begin{aligned}
\partial_{t} R_{i j k \ell}= & \partial_{t}\left(g_{\ell s} R_{i j k}^{s}\right)=\partial_{t} g_{\ell s} R_{i j k}^{s}+g_{\ell s} \partial_{t} R_{i j k}^{s} \\
= & \left(-2 R_{\ell s}+2 \alpha_{1} \nabla_{\ell} \phi(t) \nabla_{s} \phi(t)+2 \alpha_{2} \nabla_{\ell} \nabla_{s} \phi(t)\right) R_{i j k}^{s} \\
& +g_{\ell s}\left(\tilde{I}_{1}+\tilde{I}_{2}+\tilde{I}_{3}\right) \\
= & \left(-2 R_{\ell s} R_{i j k}^{s}+g_{\ell s} \tilde{I}_{1}\right) \\
& +2 \alpha_{1} R_{i j k}^{s} \nabla_{\ell} \phi(t) \nabla_{s} \phi(t)+2 \alpha_{2} R_{i j k}^{s} \nabla_{\ell} \nabla_{s} \phi(t)+g_{\ell s}\left(\tilde{I}_{2}+\tilde{I}_{3}\right) .
\end{aligned}
$$

The first bracket on the right-hand side follows from Hamilton's computation 9, 13; the rest terms can be computed from the expressions for $\tilde{I}_{2}$ and $\tilde{I}_{3}$. 
Next we compute evolution equations for $\phi(t)$.

Lemma 2.6. Under (2.4)-2.5), we have

$$
\begin{aligned}
\partial_{t}\left|\nabla_{g(t)} \phi(t)\right|_{g(t)}^{2}= & \Delta_{g(t)}\left|\nabla_{g(t)} \phi(t)\right|_{g(t)}^{2}+2 \beta_{2}\left|\nabla_{g(t)} \phi(t)\right|_{g(t)}^{2} \\
& -2\left|\nabla_{g(t)}^{2} \phi(t)\right|_{g(t)}^{2}-2 \alpha_{1}\left|\nabla_{g(t)} \phi(t)\right|_{g(t)}^{4} \\
& +\left(4 \beta_{1}-2 \alpha_{2}\right)\left\langle\nabla_{g(t)} \phi(t) \otimes \nabla_{g(t)} \phi(t), \nabla_{g(t)}^{2} \phi(t)\right\rangle_{g(t)} .
\end{aligned}
$$

Proof. Using (2.5) we have

$$
\begin{aligned}
\partial_{t} \nabla_{i} \phi(t) & =\nabla_{i} \partial_{t} \phi(t)=\nabla_{i}\left(\Delta_{g(t)} \phi(t)+\beta_{1}\left|\nabla_{g(t)} \phi(t)\right|_{g(t)}^{2}+\beta_{2} \phi(t)\right) \\
& =\Delta_{g(t)} \nabla_{i} \phi(t)-R_{i j} \nabla^{j} \phi(t)+2 \beta_{1} \nabla^{j} \phi(t) \nabla_{i} \nabla_{j} \phi(t)+\beta_{2} \nabla_{i} \phi(t),
\end{aligned}
$$

where we use the identity $\nabla_{i} \Delta_{g(t)} \phi(t)=\Delta_{g(t)} \nabla_{i} \phi(t)-R_{i j} \nabla^{j} \phi(t)$. Using 2.4 we then get

$$
\begin{aligned}
\partial_{t}\left|\nabla_{g(t)} \phi(t)\right|_{g(t)}^{2}= & \partial_{t}\left(g^{i j} \nabla_{i} \phi(t) \nabla_{j} \phi(t)\right) \\
= & \partial_{t} g^{i j} \nabla_{i} \phi(t) \nabla_{j} \phi(t)+2 g^{i j} \nabla_{j} \phi(t) \partial_{t} \nabla_{i} \phi(t) \\
= & \left(2 R^{i j}-2 \alpha_{1} \nabla^{i} \phi(t) \nabla^{j} \phi(t)-2 \alpha_{2} \nabla^{i} \nabla^{j} \phi(t)\right) \nabla_{i} \phi(t) \nabla_{j} \phi(t) \\
& +2 g^{i j} \nabla_{j} \phi(t)\left(\Delta_{g(t)} \nabla_{i} \phi(t)-R_{i k} \nabla^{k} \phi(t)\right. \\
& \left.+2 \beta_{1} \nabla^{k} \phi(t) \nabla_{i} \nabla_{k} \phi(t)+\beta_{2} \nabla_{i} \phi(t)\right) \\
= & -2 \alpha_{1}\left|\nabla_{g(t)} \phi(t)\right|_{g(t)}^{4}-2 \alpha_{2} \nabla^{i} \nabla^{j} \phi(t) \nabla_{i} \phi(t) \nabla_{j} \phi(t) \\
& +2 \nabla^{i} \phi(t) \Delta_{g(t)} \nabla_{i} \phi(t)+4 \beta_{1} \nabla_{i} \nabla_{k} \phi(t) \nabla^{i} \phi(t) \nabla^{k} \phi(t) \\
& +2 \beta_{2}\left|\nabla_{g(t)} \phi(t)\right|_{g(t)}^{2}
\end{aligned}
$$

which implies the desired equation.

Lemma 2.7. Under 2.4 (2.5), we have

$$
\begin{aligned}
\partial_{t}\left(\nabla_{i} \nabla_{j} \phi(t)\right)= & \Delta_{g(t)}\left(\nabla_{i} \nabla_{j} \phi(t)\right)+2 R_{p i j q} \nabla^{p} \nabla^{q} \phi(t)+\beta_{2} \nabla_{i} \nabla_{j} \phi(t) \\
& -R_{i p} \nabla^{p} \nabla_{j} \phi(t)-R_{j p} \nabla^{p} \nabla_{i} \phi(t)-2 \alpha_{1}\left|\nabla_{g(t)} \phi(t)\right|_{g(t)}^{2} \nabla_{i} \nabla_{j} \phi(t) \\
& +\left(2 \beta_{1}-\alpha_{2}\right) \nabla_{k} \phi(t) \nabla_{k} \nabla_{i} \nabla_{j} \phi(t)+2 \beta_{1} \nabla_{i} \nabla^{k} \phi(t) \nabla_{j} \nabla_{k} \phi(t) \\
& +2\left(\beta_{1}-\alpha_{2}\right) R_{p i j q} \nabla^{p} \phi(t) \nabla^{q} \phi(t) .
\end{aligned}
$$

Proof. Compute

$$
\begin{aligned}
\partial_{t}\left(\nabla_{i} \nabla_{j} \phi(t)\right) & =\partial_{t}\left[\partial_{i} \partial_{j} \phi(t)-\Gamma_{i j}^{k} \partial_{k} \phi(t)\right] \\
& =\partial_{i} \partial_{j}\left(\partial_{t} \phi(t)\right)-\left(\partial_{t} \Gamma_{i j}^{k}\right) \partial_{k} \phi(t)-\Gamma_{i j}^{k} \cdot \partial_{t}\left(\partial_{k} \phi(t)\right) \\
& =\nabla_{i} \nabla_{j}\left(\partial_{t} \phi(t)\right)-\partial_{t} \Gamma_{i j}^{k} \cdot \partial_{k} \phi(t) .
\end{aligned}
$$


Using 2.5 we have

$$
\begin{aligned}
\nabla_{i} \nabla_{j}\left(\partial_{t} \phi(t)\right)= & \nabla_{i} \nabla_{j}\left(\Delta_{g(t)} \phi(t)+\beta_{1}\left|\nabla_{g(t)} \phi(t)\right|_{g(t)}^{2}+\beta_{2} \phi(t)\right) \\
= & \nabla_{i}\left(\nabla_{k} \nabla_{j} \nabla^{k} \phi(t)-R_{j \ell} \nabla^{\ell} \phi(t)\right)+2 \beta_{1}\left(\nabla_{i} \nabla^{k} \phi(t) \nabla_{j} \nabla_{k} \phi(t)\right. \\
& \left.+\nabla^{k} \phi(t) \nabla_{i} \nabla_{j} \nabla_{k} \phi(t)\right)+\beta_{2} \nabla_{i} \nabla_{j} \phi(t) \\
= & \nabla_{i} \nabla_{k} \nabla_{j} \nabla^{k} \phi(t)-\nabla_{i} R_{j \ell} \nabla^{\ell} \phi(t)-R_{j \ell} \nabla_{i} \nabla^{\ell} \phi(t)+\beta_{2} \nabla_{i} \nabla_{j} \phi(t) \\
& +2 \beta_{1}\left(\nabla_{i} \nabla^{k} \phi(t) \nabla_{j} \nabla_{k} \phi(t)+\nabla^{k} \phi(t) \nabla_{i} \nabla_{j} \nabla_{k} \phi(t)\right) \\
= & \nabla_{k} \nabla_{i} \nabla_{j} \nabla^{k} \phi(t)-R_{i k j}^{\ell} \nabla_{\ell} \nabla^{k} \phi(t)+R_{i k \ell}^{k} \nabla_{j} \nabla^{\ell} \phi(t) \\
& -\nabla_{i} R_{j \ell} \nabla^{\ell} \phi(t)-R_{j \ell} \nabla_{i} \nabla^{\ell} \phi(t)+\beta_{2} \nabla_{i} \nabla_{j} \phi(t) \\
& +2 \beta_{1}\left(\nabla_{i} \nabla^{k} \phi(t) \nabla_{j} \nabla_{k} \phi(t)+\nabla^{k} \phi(t) \nabla_{i} \nabla_{j} \nabla_{k} \phi(t)\right) .
\end{aligned}
$$

Since

$$
\begin{aligned}
\nabla_{k} \nabla_{i} \nabla_{j} \nabla^{k} \phi(t) & =\nabla^{k} \nabla_{i} \nabla_{j} \nabla_{k} \phi(t)=\nabla^{k} \nabla_{i} \nabla_{k} \nabla_{j} \phi(t) \\
& =\nabla^{k}\left(\nabla_{k} \nabla_{i} \nabla_{j} \phi(t)-R_{i k j}^{\ell} \nabla_{\ell} \phi(t)\right) \\
& =\Delta_{g(t)}\left(\nabla_{i} \nabla_{j} \phi(t)\right)-\nabla^{k} R_{i k j}^{\ell} \nabla_{\ell} \phi(t)-R_{i k j}^{\ell} \nabla^{k} \nabla_{\ell} \phi(t),
\end{aligned}
$$

we have

$$
\begin{aligned}
\nabla_{i} \nabla_{j}\left(\partial_{t} \phi(t)\right)= & \Delta_{g(t)}\left(\nabla_{i} \nabla_{j} \phi(t)\right)-R_{i \ell} \nabla^{\ell} \nabla_{j} \phi(t)-R_{j \ell} \nabla^{\ell} \nabla_{i} \phi(t)+\beta_{2} \nabla_{i} \nabla_{j} \phi(t) \\
& -\left(\nabla_{i} R_{j \ell}+\nabla_{j} R_{i \ell}-\nabla_{\ell} R_{i j}\right) \nabla^{\ell} \phi(t)-2 R_{i k j}^{\ell} \nabla_{\ell} \nabla^{k} \phi(t) \\
& +2 \beta_{1}\left(\nabla_{i} \nabla^{k} \phi(t) \nabla_{j} \nabla_{k} \phi(t)+\nabla^{k} \phi(t) \nabla_{i} \nabla_{j} \nabla_{k} \phi(t)\right) .
\end{aligned}
$$

Using Lemma 2.2 implies

$$
\begin{aligned}
\partial_{t}\left(\nabla_{i} \nabla_{j} \phi(t)\right)= & \Delta_{g(t)}\left(\nabla_{i} \nabla_{j} \phi(t)\right)-2 R_{i k j \ell} \nabla^{k} \nabla^{\ell} \phi(t)-R_{i \ell} \nabla^{\ell} \nabla_{j} \phi(t) \\
& -R_{j \ell} \nabla^{\ell} \nabla_{i} \phi(t)+\beta_{2} \nabla_{i} \nabla_{j} \phi(t)+2 \beta_{1} \nabla_{i} \nabla^{k} \phi(t) \nabla_{j} \nabla_{k} \phi(t) \\
& +2 \beta_{1} \nabla^{k} \phi(t) \nabla_{i} \nabla_{j} \nabla_{k} \phi(t)-2 \alpha_{1}\left|\nabla_{g(t)} \phi(t)\right|_{g(t)}^{2} \nabla_{i} \nabla_{j} \phi(t) \\
& -\alpha_{2} \nabla^{k} \phi(t) \nabla_{k} \nabla_{i} \nabla_{j} \phi(t)+2 \alpha_{2} R_{i k j \ell} \nabla^{\ell} \phi(t) \nabla^{k} \phi(t) .
\end{aligned}
$$

Now Lemma 2.7 follows from $\nabla_{i} \nabla_{j} \nabla_{k} \phi(t)=\nabla_{k} \nabla_{i} \nabla_{j} \phi(t)-R_{i k j}^{\ell} \nabla_{\ell} \phi(t)$.

Lemma 2.8. Under (2.4)-2.5), we have

$$
\begin{aligned}
\partial_{t}\left(\nabla_{i} \phi(t) \nabla_{j} \phi(t)\right)= & \Delta_{g(t)}\left(\nabla_{i} \phi(t) \nabla_{j} \phi(t)\right)-\nabla^{k} \phi(t)\left(R_{i k} \nabla_{j} \phi(t)+R_{j k} \nabla_{i} \phi(t)\right) \\
& -2 \nabla_{i} \nabla^{k} \phi(t) \nabla_{j} \nabla_{k} \phi(t)+2 \beta_{2} \nabla_{i} \phi(t) \nabla_{j} \phi(t) \\
& +2 \beta_{1} \nabla^{k} \phi(t)\left(\nabla_{i} \phi(t) \nabla_{j} \nabla_{k} \phi(t)+\nabla_{j} \phi(t) \nabla_{i} \nabla_{k} \phi(t)\right) .
\end{aligned}
$$

Proof. From the evolution equation for $\nabla_{i} \phi(t)$ obtained in the proof of Lemma 2.6 . we get

$$
\begin{aligned}
\partial_{t}\left(\nabla_{i} \phi(t) \nabla_{j} \phi(t)\right)= & \nabla_{j} \phi(t)\left(\Delta_{g(t)} \nabla_{i} \phi(t)-R_{i k} \nabla^{k} \phi(t)+2 \beta_{1} \nabla^{k} \phi(t) \nabla_{i} \nabla_{k} \phi(t)\right. \\
& \left.+\beta_{2} \nabla_{i} \phi(t)\right)+\nabla_{i} \phi(t)\left(\Delta_{g(t)} \nabla_{j}(t)-R_{j k} \nabla^{k} \phi(t)\right. \\
& \left.+2 \beta_{1} \nabla^{k} \phi(t) \nabla_{j} \nabla_{k} \phi(t)+\beta_{2} \nabla_{j} \phi(t)\right)
\end{aligned}
$$

which implies the equation. 
2.3. Regular flows on compact manifolds. Let $(g(t), \phi(t))_{t \in[0, T)}$ be the solution of 2.4 2.5 on a compact $m$-manifold $M$ with the initial value $(\tilde{g}, \tilde{\phi})$. Define

$$
\tilde{c}:=\max _{M}\left|\nabla_{\tilde{g}} \tilde{\phi}\right|_{\tilde{g}}^{2}, \quad D:=\frac{1}{4}\left|2 \beta_{1}-\alpha_{2}\right|^{2}-\alpha_{1} .
$$

Proposition 2.9. Suppose $(g(t), \phi(t))_{t \in[0, T)}$ is the solution of (2.4)-2.5) on a compact $m$-manifold $M$ with the initial value $(\tilde{g}, \tilde{\phi})$. Then we have

(1) Case 1: $4 \beta_{1}-2 \alpha_{2}=0$.

(1.1) If $\alpha_{1}>0$ and $\beta_{2}>0$, then

$$
\left|\nabla_{g(t)} \phi(t)\right|_{g(t)}^{2} \leq \frac{\tilde{c} \beta_{2} e^{2 \beta_{2} t}}{\tilde{c} \alpha_{1} e^{2 \beta_{2} t}+\left(\beta_{2}-\tilde{c} \alpha_{1}\right)} .
$$

(1.2) If $\alpha_{1}>0$ and $\beta_{2} \leq 0$, then

$$
\left|\nabla_{g(t)} \phi(t)\right|_{g(t)}^{2} \leq \tilde{c}
$$

(1.3) If $\alpha_{1}=0$, then

$$
\left|\nabla_{g(t)} \phi(t)\right|_{g(t)}^{2} \leq \tilde{c} e^{2 \beta_{2} t}
$$

(1.4) If $\alpha_{1}<0$ and $\beta_{2} \leq 0$, then

$$
\left|\nabla_{g(t)} \phi(t)\right|_{g(t)}^{2} \leq \frac{\tilde{c}}{1+2 \alpha_{1} \tilde{c} t} .
$$

(1.5) If $\alpha_{1}<0$ and $\beta_{2}>0$, then

$$
\left|\nabla_{g(t)} \phi(t)\right|_{g(t)}^{2} \leq \frac{\tilde{c} \beta_{2} e^{2 \beta_{2} t}}{\beta_{2}+\tilde{c} \alpha_{1}\left(e^{2 \beta_{2} t}-1\right)} .
$$

(2) Case 2: $4 \beta_{1}-2 \alpha_{2} \neq 0$.

(2.1) If $D<0$ and $\beta_{2}>0$, then

$$
\left|\nabla_{g(t)} \phi(t)\right|_{g(t)}^{2} \leq \frac{\tilde{c} \beta_{2} e^{2 \beta_{2} t}}{-\tilde{c} D e^{2 \beta_{2} t}+\left(\beta_{2}+\tilde{c} D\right)} .
$$

(2.2) If $D<0$ and $\beta_{2} \leq 0$, then

$$
\left|\nabla_{g(t)} \phi(t)\right|_{g(t)}^{2} \leq \tilde{c} .
$$

(2.3) If $D=0$, then

$$
\left|\nabla_{g(t)} \phi(t)\right|_{g(t)}^{2} \leq \tilde{c} e^{2 \beta_{2} t} .
$$

(2.4) If $D>0$ and $\beta_{2} \leq 0$, then

$$
\left|\nabla_{g(t)} \phi(t)\right|_{g(t)}^{2} \leq \frac{\tilde{c}}{1-2 D \tilde{c} t} .
$$

(2.5) If $D>$ and $\beta_{2}>0$, then

$$
\left|\nabla_{g(t)} \phi(t)\right|_{g(t)}^{2} \leq \frac{\tilde{c} \beta_{2} e^{2 \beta_{2} t}}{\beta_{2}-\tilde{c} D\left(e^{2 \beta_{2} t}-1\right)} .
$$

Proof. For any time $t$, we have

$$
\left\langle\nabla_{g(t)} \phi(t) \otimes \nabla_{g(t)} \phi(t), \nabla_{g(t)}^{2} \phi(t)\right\rangle_{g(t)} \leq\left|\nabla_{g(t)} \phi(t)\right|_{g(t)}^{2}\left|\nabla_{g(t)}^{2} \phi(t)\right|_{g(t)} .
$$


By Lemma 2.6 we have

$$
\begin{aligned}
\partial_{t}\left|\nabla_{g(t)} \phi(t)\right|_{g(t)}^{2} \leq & \Delta_{g(t)}\left|\nabla_{g(t)} \phi(t)\right|_{g(t)}^{2}+2 \beta_{2}\left|\nabla_{g(t)} \phi(t)\right|_{g(t)}^{2} \\
& -2\left|\nabla_{g(t)}^{2} \phi(t)\right|_{g(t)}^{2}-2 \alpha_{1}\left|\nabla_{g(t)} \phi(t)\right|_{g(t)}^{4} \\
& +\left|4 \beta_{1}-2 \alpha_{2}\right|\left|\nabla_{g(t)} \phi(t)\right|^{2}\left|\nabla_{g(t)}^{2} \phi(t)\right| .
\end{aligned}
$$

For convenience, set

$$
u(t):=\left|\nabla_{g(t)} \phi(t)\right|_{g(t)}^{2}, \quad v(t):=\left|\nabla_{g(t)}^{2} \phi(t)\right|_{g(t)} .
$$

Then

$$
\partial_{t} u(t) \leq \Delta_{g(t)} u(t)+2 \beta_{2} u(t)-2 v^{2}(t)-2 \alpha_{1} u^{2}(t)+\left|4 \beta_{1}-2 \alpha_{2}\right| u(t) v(t) .
$$

(1) Case 1: $4 \beta_{1}-2 \alpha_{2}=0$. In this case, the above inequality becomes

$$
\partial_{t} u(t) \leq \Delta_{g(t)} u(t)+2 \beta_{2} u(t)-2 \alpha_{1} u^{2}(t) .
$$

If $\alpha_{1} \geq 0$, then

$$
\partial_{t} u(t) \leq \Delta_{g(t)} u(t)+2 \beta_{2} u(t)
$$

and

$$
\partial_{t}\left(e^{-2 \beta_{2} t} u(t)\right)=e^{-2 \beta_{2} t}\left(-2 \beta_{2} u(t)+\partial_{t} u(t)\right) \leq \Delta\left(e^{-2 \beta_{2} t} u(t)\right)
$$

from which we obtain $u(t) \leq \tilde{c} e^{2 \beta_{2} t}$ by the maximum principle.

If $\alpha_{1}<0$ and $\beta_{2} \leq 0$, then

$$
\partial_{t} u(t) \leq \Delta_{g(t)} u(t)-2 \alpha_{1} u^{2}(t)
$$

and $u_{t} \leq \frac{u(0)}{1+2 \alpha_{1} u(0) t} \leq \frac{\tilde{c}}{1+2 \alpha_{1} \tilde{c} t}$. On the other hand, if $\beta_{2}>0$, then

$$
u(t) \leq \frac{\tilde{c} \beta_{2} e^{2 \beta_{2} t}}{\beta_{2}+\tilde{c} \alpha_{1}\left(e^{2 \beta_{2} t}-1\right)}
$$

since the solution to the ordinary differential equation

is of the form

$$
\frac{d U(t)}{d t}=2 \beta_{2} U(t)-2 \alpha_{1} U^{2}(t), \quad U(0)=\tilde{c}
$$

$$
U(t)=\frac{\tilde{c} \beta_{2} e^{2 \beta_{2} t}}{\beta_{2}+\tilde{c} \alpha_{1}\left(e^{2 \beta_{2} t}-1\right)} .
$$

(2) Case 2: $4 \beta_{1}-2 \alpha_{2} \neq 0$. Using the inequality $a b \leq \epsilon a^{2}+\frac{1}{4 \epsilon} b^{2}$ for any nonnegative numbers $a, b$ and any positive number $\epsilon$, we obtain

$$
\begin{aligned}
\partial_{t} u(t) \leq & \Delta_{g(t)} u(t)+2 \beta_{2} u(t)-2 v^{2}(t)-2 \alpha_{1} u^{2}(t) \\
& +\left|4 \beta_{1}-2 \alpha_{2}\right|\left(\epsilon v^{2}(t)+\frac{1}{4 \epsilon} u^{2}\right) \\
= & \Delta_{g(t)} u(t)+2 \beta_{2} u(t)-\left(\epsilon\left|4 \beta_{1}-2 \alpha_{2}\right|-2\right) v^{2}(t) \\
& +\left(\frac{\left|4 \beta_{1}-2 \alpha_{2}\right|}{4 \epsilon}-2 \alpha_{1}\right) u^{2}(t) .
\end{aligned}
$$

Choosing $\epsilon:=2 /\left|4 \beta_{1}-2 \alpha_{2}\right|$ implies

$$
\partial_{t} u(t) \leq \Delta_{g(t)} u(t)+2 \beta_{2} u(t)+2 D u^{2}(t),
$$

where $D$ is given in 2.7). This is just the case (1) if we replace $\alpha_{1}$ by $-D$. The following discussion can be obtained. 
Corollary 2.10. Suppose $(g(t), \phi(t))_{t \in[0, T)}$ is the solution of (2.4)-2.5) on a compact $m$-manifold $M$ with the initial value $(\tilde{g}, \tilde{\phi})$. If $\alpha_{1}, \alpha_{2}, \beta_{1}, \beta_{2}$ satisfy one of the conditions

(i) $\beta_{2} \leq 0$ and $4 \alpha_{1} \geq\left(2 \beta_{1}-\alpha_{2}\right)^{2}$, or

(ii) $\beta_{2}>0$ and $\frac{4}{\tilde{\tilde{c}}} \beta_{2}+\left|2 \beta_{1}-\alpha_{2}\right|^{2} \geq 4 \alpha_{1}>\left(2 \beta_{1}-\alpha_{2}\right)^{2}$, then

$$
\left|\nabla_{g(t)} \phi(t)\right|_{g(t)}^{2} \leq C
$$

on $M \times[0, T)$, where $C$ is a positive constant depending only on $\alpha_{1}, \beta_{2}$, and $\left|\nabla_{\tilde{g}} \tilde{\phi}\right|_{\tilde{g}}^{2}$.

In particular, we recover List's result 19 for $\left(\alpha_{1}, \alpha_{2}, \beta_{1}, \beta_{2}\right)=(4,0,0,0)$. Anther example is $\left(\alpha_{1}, \alpha_{2}, \beta_{1}, \beta_{2}\right)=\left(\alpha_{1}, 2 \beta_{1}, \beta_{1}, 0\right)$, where $\alpha_{1} \geq 0$ and $\beta_{1} \in \mathbf{R}$.

Definition 2.11. We say the flow (2.4)-2.5) is regular, if the constants $\alpha_{1}, \alpha_{2}, \beta_{1}, \beta_{2}$ satisfy the conditions (i) or (ii) in Corollary 2.10 . An $\left(\alpha_{1}, \alpha_{2}, \beta_{1}, \beta_{2}\right)$-flow is $\star$-regular if the associated $\left(\alpha_{1}, 0, \beta_{1}-\alpha_{2}, \beta_{2}\right)$-flow (see Proposition 2.12 is regular.

Clearly that there are no relations between regular flows and $\star$-regular in general. For example, $(1,1,0,0)$-flow is regular but not $\star$-regular, while $(1,1,2,0)$-flow is $\star$ regular but not regular.

2.4. Reduction to $\left(\alpha_{1}, 0, \beta_{1}, \beta_{2}\right)$-flow. Let $(\bar{g}(t), \bar{\phi}(t))$ be the solution of 2.4 2.5 ; that is,

$$
\begin{aligned}
\partial_{t} \bar{g}(t) & =-2 \operatorname{Ric}_{\bar{g}(t)}+2 \alpha_{1} \nabla_{\bar{g}(t)} \bar{\phi}(t) \otimes \nabla_{\bar{g}(t)} \bar{\phi}(t)+2 \alpha_{2} \nabla_{\bar{g}(t)}^{2} \bar{\phi}(t), \\
\partial_{t} \bar{\phi}(t) & =\Delta_{\bar{g}(t)} \bar{\phi}(t)+\beta_{1}\left|\nabla_{\bar{g}(t)} \bar{\phi}(t)\right|_{\bar{g}(t)}^{2}+\beta_{2} \bar{\phi}(t) .
\end{aligned}
$$

Consider a 1-parameter family of diffeomorphisms $\Phi(t): M \rightarrow M$ by

$$
\frac{d}{d t} \Phi(t)=-\alpha_{2} \nabla_{\bar{g}(t)} \bar{\phi}(t), \quad \Phi(0)=\operatorname{Id}_{M} .
$$

The above system of ODE is always solvable. Define

$$
g(t):=[\Phi(t)]^{*} \bar{g}(t), \quad \phi(t):=[\Phi(t)]^{*} \bar{\phi}(t) .
$$

Then

$$
\begin{aligned}
\partial_{t} g(t)= & {[\Phi(t)]^{*}\left(\partial_{t} \bar{g}(t)\right)-\alpha_{2}[\Phi(t)]^{*}\left(\mathscr{L}_{\nabla_{\bar{g}(t)} \bar{\phi}(t)} \bar{g}(t)\right) } \\
= & {[\Phi(t)]^{*}\left(-2 \operatorname{Ric}_{\bar{g}(t)}+2 \alpha_{1} \nabla_{\bar{g}(t)} \bar{\phi}(t) \otimes \nabla_{\bar{g}(t)} \bar{\phi}(t)+2 \alpha_{2} \nabla_{\bar{g}(t)}^{2} \bar{\phi}(t)\right) } \\
& -2 \alpha_{2}[\Phi(t)]^{*} \nabla_{\bar{g}(t)}^{2} \bar{\phi}(t) \\
= & -2 \operatorname{Ric}_{g(t)}+2 \alpha_{1} \nabla_{g(t)} \phi(t) \otimes \nabla_{g(t)} \phi(t), \\
\partial_{t} \phi(t)= & \partial_{t}\left([\Phi(t)]^{*} \bar{\phi}(t)\right) \\
= & \partial_{t}(\bar{\phi}(t) \circ \Phi(t)) \\
= & \partial_{t} \bar{\phi}(t) \circ \Phi(t)-\alpha_{2}\left|\nabla_{\bar{g}(t)} \bar{\phi}(t)\right|_{\bar{g}(t)}^{2} \\
= & \Delta_{g(t)} \phi(t)+\left(\beta_{1}-\alpha_{2}\right)\left|\nabla_{g(t)} \phi(t)\right|_{g(t)}^{2}+\beta_{2} \phi(t) .
\end{aligned}
$$

Proposition 2.12. Under a 1-parameter family of diffeomorphisms given by 2.9, any solution of an $\left(\alpha_{1}, \alpha_{2}, \beta_{1}, \beta_{2}\right)$-flow is equivalent to a solution of $\left(\alpha_{1}, 0, \beta_{1}-\right.$ $\left.\alpha_{2}, \beta_{2}\right)$ - flow. 
2.5. De Turck's trick. By Proposition 2.12, we suffice to study $\left(\alpha_{1}, 0, \beta_{1}, \beta_{2}\right)$-flow:

$$
\begin{aligned}
& \partial_{t} g(t)=-2 \operatorname{Ric}_{g(t)}+2 \alpha_{1} \nabla_{g(t)} \phi(t) \otimes \nabla_{g(t)} \phi(t), \\
& \partial_{t} \phi(t)=\Delta_{g(t)} \phi(t)+\beta_{1}\left|\nabla_{g(t)} \phi(t)\right|_{g(t)}^{2}+\beta_{2} \phi(t) .
\end{aligned}
$$

Let $(M, \tilde{g})$ be an $m$-dimensional compact or complete noncompact Riemannian manifold with $\tilde{g}=\tilde{g}_{i j} d x^{i} \otimes d x^{j}$ and $\tilde{\phi}$ a smooth function on $M$. Let $(\hat{g}(t), \hat{\phi}(t))_{t \in[0, T]}$ be a solution of 2.11-2.12 with the initial data $(\tilde{g}, \tilde{\phi})$, that is,

$$
\begin{aligned}
& \partial_{t} \hat{g}(t)=-2 \operatorname{Ric}_{\hat{g}(t)}+2 \alpha_{1} \nabla_{\hat{g}(t)} \hat{\phi}(t) \otimes \nabla_{\hat{g}(t)} \hat{\phi}(t), \\
& \partial_{t} \hat{\phi}(t)=\Delta_{\hat{g}(t)} \hat{\phi}(t)+\beta_{1}\left|\nabla_{\hat{g}(t)} \hat{\phi}(t)\right|_{\hat{g}(t)}^{2}+\beta_{2} \hat{\phi}(t)
\end{aligned}
$$

with $(\hat{g}(0), \hat{\phi}(0))=(\tilde{g}, \tilde{\phi})$.

Notation 2.13. If $\hat{g}(t)$ is a time-dependent Riemannian metric, its components are written as $\hat{g}_{i j}$ or $\hat{g}_{i j}(x, t)$ when we want to indicate space and time. The corresponding components of $\operatorname{Rm}_{\hat{g}(t)}, \operatorname{Ric}_{\hat{g}(t)}$ and $\nabla_{\hat{g}(t)}$ are $\hat{R}_{i j k \ell}, \hat{R}_{i j}$ and $\hat{\nabla}_{i}$, respectively. In the form of local components, we always omit space and time variables for convenience.

Locally, the system 2.13 2.14 is of the form

$$
\partial_{t} \hat{g}_{i j}=-2 \hat{R}_{i j}+2 \alpha_{1} \hat{\nabla}_{i} \hat{\phi} \hat{\nabla}_{j} \hat{\phi}, \quad \partial_{t} \hat{\phi}=\hat{\Delta} \hat{\phi}+\beta_{1}|\hat{\nabla} \hat{\phi}|_{\hat{g}}^{2}+\beta_{2} \hat{\phi}
$$

with $\left(\hat{g}_{i j}(0), \hat{\phi}(0)\right)=\left(\tilde{g}_{i j}, \tilde{\phi}\right)$. The system 2.15 is not strictly parabolic even for the case $\alpha_{1}=\beta_{1}=\beta_{2}$. As in the Ricci flow (see [26]) we consider one-parameter family of diffeomorphisms $\left(\Psi_{t}\right)_{t \in[0, T]}$ on $M$ as follows: Let

$$
g(t):=\Psi_{t}^{*} \hat{g}(t)=g_{i j}(x, t) d x^{i} \otimes d x^{j}, \quad \phi(t):=\Psi_{t}^{*} \hat{\phi}(t), \quad t \in[0, T]
$$

and $\Psi_{t}(x):=y(x, t)$ be the solution of the quasilinear first order system

$$
\partial_{t} y^{\alpha}=\frac{\partial}{\partial x^{k}} y^{\alpha} \cdot g^{\beta \gamma}\left(\Gamma_{\beta \gamma}^{k}-\tilde{\Gamma}_{\beta \gamma}^{k}\right), \quad y^{\alpha}(x, 0)=x^{\alpha},
$$

where $\Gamma$ and $\tilde{\Gamma}$ are Christoffel symbols of $g$ and $\hat{g}$ respectively. As in [19, 26], we have

$$
\partial_{t} g_{i j}=-2 R_{i j}+2 \alpha_{1} \nabla_{i} \phi \nabla_{j} \phi+\nabla_{i} V_{j}+\nabla_{j} V_{i},
$$

where

$$
V_{i}:=g_{i k} g^{\beta \gamma}\left(\Gamma_{\beta \gamma}^{k}-\tilde{\Gamma}_{\beta \gamma}^{k}\right)
$$


Similarly, we have

$$
\begin{aligned}
\partial_{t} \phi(x, t)= & \partial_{t} \hat{\phi}(y, t)=\partial_{t} \hat{\phi}(y, t)+\frac{\partial}{\partial y^{\alpha}} \hat{\phi}(y, t) \partial_{t} y^{\alpha} \\
= & \left(\hat{g}^{\alpha \beta}(y, t) \hat{\nabla}_{\alpha} \hat{\nabla}_{\beta} \hat{\phi}(y, t)+\beta_{1} \hat{g}^{\alpha \beta}(y, t) \hat{\nabla}_{\alpha} \hat{\phi}(y, t) \hat{\nabla}_{\beta} \hat{\phi}(y, t)+\beta_{2} \hat{\phi}(y, t)\right) \\
& +\frac{\partial}{\partial y^{\alpha}} \hat{\phi}(y, t) \frac{\partial y^{\alpha}}{\partial x^{k}} g^{i k} V_{i} \\
= & \frac{\partial}{\partial x^{k}} \hat{\phi}(y, t) \cdot g^{i k} V_{i}+\beta_{1} g^{i j} \frac{\partial y^{\alpha}}{\partial x^{i}} \frac{\partial y^{\beta}}{\partial x^{j}} \hat{\nabla}_{\alpha} \hat{\phi}(y, t) \hat{\nabla}_{\beta} \hat{\phi}(y, t) \\
& +\beta_{2} \hat{\phi}(y, t)+g^{i j} \frac{\partial y^{\alpha}}{\partial x^{i}} \frac{\partial y^{\beta}}{\partial x^{j}} \hat{\nabla}_{\alpha} \hat{\nabla}_{\beta} \hat{\phi}(y, t) \\
= & \Delta \phi(x, t)+\beta_{1}|\nabla \phi|_{g}^{2}+\beta_{2} \phi(x, t)+\langle V, \nabla \phi\rangle_{g} .
\end{aligned}
$$

Here, $\Delta$ and $\nabla$ are Laplacian and Levi-Civita connection of $g$ accordingly. Hence, under the one-parameter family of diffeomorphisms $\left(\Psi_{t}\right)_{t \in[0, T]}$ on $M, 2.15$ is equivalent to

$$
\begin{aligned}
\partial_{t} g_{i j} & =-2 R_{i j}+2 \alpha_{1} \nabla_{i} \phi \nabla_{j} \phi+\nabla_{i} V_{j}+\nabla_{j} V_{i} \\
\partial_{t} \phi & =\Delta \phi+\beta_{1}|\nabla \phi|_{g}^{2}+\beta_{2} \phi+\langle V, \nabla \phi\rangle_{g}
\end{aligned}
$$

with $\left(g_{i j}(0), \phi(0)\right)=\left(\tilde{g}_{i j}, \tilde{\phi}\right)$.

Lemma 2.14. the system (2.19) 2.20) is strictly parabolic. Moreover

$$
\begin{aligned}
\partial_{t} g_{i j}= & g^{\alpha \beta} \tilde{\nabla}_{\alpha} \tilde{\nabla}_{\beta} g_{i j}+g^{\alpha \beta} g_{i p} \tilde{g}^{p q} \tilde{R}_{j \alpha q \beta}+g^{\alpha \beta} g_{j p} \tilde{g}^{p q} \tilde{R}_{i \alpha q \beta} \\
& +\frac{1}{2} g^{\alpha \beta} g^{p q}\left(\tilde{\nabla}_{i} g_{p \alpha} \tilde{\nabla}_{j} g_{q \beta}+2 \tilde{\nabla}_{\alpha} g_{j p} \tilde{\nabla}_{q} g_{i \beta}-2 \tilde{\nabla}_{\alpha} g_{j p} \tilde{\nabla}_{\beta} g_{i q}\right. \\
& \left.-2 \tilde{\nabla}_{j} g_{p \alpha} \tilde{\nabla}_{\beta} g_{i q}-2 \tilde{\nabla}_{i} g_{p \alpha} \tilde{\nabla}_{\beta} g_{j q}\right)+2 \alpha_{1} \tilde{\nabla}_{i} \phi \tilde{\nabla}_{j} \phi, \\
\partial_{t} \phi= & g^{i j} \tilde{\nabla}_{i} \tilde{\nabla}_{j} \phi+\beta_{1}|\tilde{\nabla} \phi|_{g}^{2}+\beta_{2} \phi .
\end{aligned}
$$

Proof. The first equation 2.21) directly follows from the computations made in $[19,26]$ and the only difference is the sign of the Riemann curvature tensors used in this paper. To 2.22, we first observe that $\Delta \phi+\langle V, \nabla \phi\rangle_{g}=g^{i j} \tilde{\nabla}_{i} \tilde{\nabla}_{j} \phi$ as showed in 19; then

$$
\partial_{t} \phi=g^{i j} \tilde{\nabla}_{i} \tilde{\nabla}_{j} \phi+\beta_{1} g^{i j} \tilde{\nabla}_{i} \phi \tilde{\nabla}_{j} \phi+\beta_{2} \phi
$$

since $\nabla \phi=d \phi=\tilde{\nabla} \phi$.

\section{Complete And nONCOMPACt CASE}

In this section we study the flow (2.4)- 2.5 on complete and noncompact Riemannian manifolds. The main result of this paper is

Theorem 3.1. Let $(M, g)$ be an m-dimensional complete and noncompact Riemannian manifold with $\left|\mathrm{Rm}_{g}\right|_{g}^{2} \leq k_{0}$ on $M$, where $k_{0}$ is a positive constant, and let $\phi$ be a smooth function on $M$ satisfying $|\phi|^{2}+\left|\nabla_{g} \phi\right|_{g}^{2} \leq k_{1}$ and $\left|\nabla_{g}^{2} \phi\right|_{g}^{2} \leq k_{2}$. Then there exists a constant $T=T\left(m, k_{0}, k_{1}\right)>0$, depending only on $m$ and $k_{0}, k_{1}$, such that any $\star$-regular $\left(\alpha_{1}, \alpha_{2}, \beta_{1}, \beta_{2}\right)$-flow 2.4 -2.5) has a smooth solution $(g(t), \phi(t))$ 
on $M \times[0, T]$ and satisfies the following curvature estimate. For any nonnegative integer $n$, there exist constants $C_{k}>0$, depending only on $m, n, k_{0}, k_{1}, k_{2}$, such that

$$
\left|\nabla_{g(t)}^{k} \operatorname{Rm}_{g(t)}\right|_{g(t)}^{2} \leq \frac{C_{k}}{t^{k}}, \quad\left|\nabla_{g(t)}^{k} \phi(t)\right|_{g(t)}^{2} \leq \frac{C_{k}}{t^{?}}
$$

on $M \times[0, T]$.

By Proposition 2.12 we suffice to study a regular $\left(\alpha_{1}, 0, \beta_{1}, \beta_{2}\right)$-flow:

$$
\begin{aligned}
& \partial_{t} \hat{g}(t)=-2 \operatorname{Ric}_{\hat{g}(t)}+2 \alpha_{1} \nabla_{\hat{g}(t)} \hat{\phi}(t) \otimes \nabla_{\hat{g}(t)} \hat{\phi}(t), \\
& \partial_{t} \hat{\phi}(t)=\Delta_{\hat{g}(t)} \hat{\phi}(t)+\beta_{1}\left|\nabla_{\hat{g}(t)} \hat{\phi}(t)\right|_{\hat{g}(t)}^{2}+\beta_{2} \hat{\phi}(t),
\end{aligned}
$$

where $(\tilde{g}, \tilde{\phi})=(\hat{g}(0), \hat{\phi}(0))$ is a fixed pair consisting of a Riemannian metric $\hat{g}$ and a smooth function $\hat{\phi}$. According to De Turck's trick, the above system of parabolic partial differential equations are reduced to 2.10)-2.20.

Suppose that $D \subset M$ is a domain with boundary $\partial D$ a compact smooth $(m-1)$ dimensional submanifold of $M$, and the closure $\bar{D}:=D \cup \partial D$ is a compact subset of $M$. We shall shove the following Dirichlet boundary problem:

$$
\begin{aligned}
\partial_{t} g_{i j} & =-2 R_{i j}+2 \alpha_{1} \nabla_{i} \phi \nabla_{j} \phi+\nabla_{i} V_{j}+\nabla_{j} V_{i}, \quad \text { in } D \times[0, T], \\
\partial_{t} \phi & =\Delta \phi+\beta_{1}|\nabla \phi|_{g}^{2}+\beta_{2} \phi+\langle V, \nabla \phi\rangle_{g}, \quad \text { in } D \times[0, T], \\
\left(g_{i j}, \phi\right) & =\left(\tilde{g}_{i j}, \tilde{\phi}\right), \quad \text { on } D_{T} .
\end{aligned}
$$

where

$$
D_{T}:=(D \times\{0\}) \cup(\partial D \cup[0, T])
$$

stands for the parabolic boundary of the domain $D \times[0, T]$.

Consider the assumption

$$
\left|\operatorname{Rm}_{\tilde{g}}\right|_{\tilde{g}}^{2} \leq k_{0}, \quad\left|\nabla_{\tilde{g}} \tilde{\phi}\right|_{\tilde{\phi}}^{2} \leq k_{1} .
$$

3.1. Zeroth order estimates. Suppose that $\left(g_{i j}, \phi\right)$ is a solution of $(3.2)$. For each positive integer $n$, define

$$
u=u(x, t):=g^{\alpha_{1} \beta_{1}} \tilde{g}_{\beta_{1} \alpha_{2}} g^{\alpha_{2} \beta_{2}} \tilde{g}_{\beta_{2} \alpha_{3}} \cdots g^{\alpha_{n} \beta_{n}} \tilde{g}_{\beta_{n} \alpha_{1}}
$$

on $D \times[0, T]$.

Lemma 3.2. If $\left|\mathrm{Rm}_{\tilde{g}}\right|_{\tilde{g}}^{2} \leq k_{0}$, then the function $u=u(x, t)$ satisfies

$$
\partial_{t} u \leq g^{\alpha \beta} \tilde{\nabla}_{\alpha} \tilde{\nabla}_{\beta} u+2 n m \sqrt{k_{0}} u^{1+\frac{1}{n}} \quad \text { in } D \times[0, T], \quad u=m \quad \text { on } D_{T} .
$$

Proof. The proof is identically similar to that of [19, 26]; for completeness, we give a self-contained proof. Since $\partial_{t} g^{i j}=-g^{i k} g^{j \ell} \partial_{t} g_{k \ell}$ and $\tilde{\nabla}_{\beta} g^{i j}=-g^{i p} g^{j q} \tilde{\nabla}_{\beta} g_{p q}$, it follows from (2.21) that

$$
\begin{aligned}
\partial_{t} g^{i j}= & g^{\alpha \beta} \tilde{\nabla}_{\alpha} \tilde{\nabla}_{\beta} g^{i j}+g^{\alpha \beta} g^{j \ell} g^{i k} \tilde{\nabla}_{\alpha} g^{i k} \tilde{\nabla}_{\beta} g_{k \ell}+g^{\alpha \beta} g^{i k} \tilde{\nabla}_{\alpha} g^{j \ell} \tilde{\nabla}_{\beta} g_{k \ell} \\
& -g^{\alpha \beta} g^{i k} g^{j \ell} g_{k p} \tilde{g}^{p q} \tilde{R}_{\ell \alpha q \beta}-g^{\alpha \beta} g^{i k} g^{j \ell} g_{p \ell} \tilde{g}^{p q} \tilde{R}_{k \alpha q \beta}-2 \alpha_{1} g^{i k} g^{j \ell} \tilde{\nabla}_{k} \phi \tilde{\nabla}_{\ell} \phi \\
& +\frac{1}{2} g^{\alpha \beta} g^{p q} g^{j \ell}\left(2 \tilde{\nabla}_{\alpha} g_{p \ell} \tilde{\nabla}_{\beta} g_{k q}+2 \tilde{\nabla}_{\ell} g_{p \alpha} \tilde{\nabla}_{\beta} g_{k q}+2 \tilde{\nabla}_{k} g_{p \alpha} \tilde{\nabla}_{\beta} g_{q \ell}\right. \\
& \left.-2 \tilde{\nabla}_{\alpha} g_{\ell p} \tilde{\nabla}_{q} g_{k \beta}-\tilde{\nabla}_{k} g_{p \alpha} \tilde{\nabla}_{\ell} g_{q \beta}\right) .
\end{aligned}
$$


Choosing a normal coordinate system such that

$$
\tilde{g}_{i j}=\delta_{i j}, \quad g_{i j}=\lambda_{i} \delta_{i j},
$$

we conclude from (3.7) that

$$
\begin{aligned}
\partial_{t} g^{i j}= & g^{\alpha \beta} \tilde{\nabla}_{\alpha} \tilde{\nabla}_{\beta} g^{i j}-\frac{2}{\lambda_{i} \lambda_{j} \lambda_{k} \lambda_{\ell}} \tilde{\nabla}_{\ell} g_{i k} \tilde{\nabla}_{\ell} g_{j k}-\frac{1}{\lambda_{i} \lambda_{k}} \tilde{R}_{i k j k}-\frac{1}{\lambda_{j} \lambda_{k}} \tilde{R}_{i k j k} \\
& +\frac{1}{2 \lambda_{i} \lambda_{j} \lambda_{k} \lambda_{\ell}}\left(2 \tilde{\nabla}_{k} g_{\ell j} \tilde{\nabla}_{k} g_{i \ell}+2 \tilde{\nabla}_{j} g_{\ell k} \tilde{\nabla}_{k} g_{i \ell}+2 \tilde{\nabla}_{i} g_{\ell k} \tilde{\nabla}_{k} g_{\ell j}\right. \\
& \left.-2 \tilde{\nabla}_{k} g_{j \ell} \tilde{\nabla}_{\ell} g_{i k}-\tilde{\nabla}_{i} g_{\ell k} \tilde{\nabla}_{j} g_{\ell k}\right)-\frac{2 \alpha_{1}}{\lambda_{i} \lambda_{j}} \tilde{\nabla}_{i} \phi \tilde{\nabla}_{j} \phi .
\end{aligned}
$$

From $u=\sum_{i=1}^{n}\left(1 / \lambda_{i}\right)^{n}$, we obtain

$$
\begin{aligned}
& \partial_{t} u=n\left(\frac{1}{\lambda_{i}}\right)^{n-1} \partial_{t} g^{i i} \\
& =\left(\frac{n}{\lambda_{i}^{n-1}} g^{\alpha \beta} \tilde{\nabla}_{\alpha} \tilde{\nabla}_{\beta} g^{i i}-\frac{2 n}{\lambda_{i}^{n} \lambda_{k}} \tilde{R}_{i k i k}-\frac{n}{\lambda_{i}^{n+1} \lambda_{k} \lambda_{\ell}} \tilde{\nabla}_{\ell} g_{i k} \tilde{\nabla}_{\ell} g_{i k}\right. \\
& +\frac{n}{2 \lambda_{i}^{n+1} \lambda_{k} \lambda_{\ell}}\left(4 \tilde{\nabla}_{i} g_{\ell k} \tilde{\nabla}_{k} g_{i \ell}-2 \tilde{\nabla}_{k} g_{i \ell} \tilde{\nabla}_{\ell} g_{i k}-\tilde{\nabla}_{i} g_{\ell k} \tilde{\nabla}_{i} g_{\ell k}\right) \\
& \left.-\frac{2 \alpha_{1} n}{\lambda_{i}^{n+1}}\left|\nabla_{\tilde{g}} \phi\right|_{\tilde{g}}^{2}\right) \\
& =\frac{n}{\lambda_{i}^{n-1}} g^{\alpha \beta} \tilde{\nabla}_{\alpha} \tilde{\nabla}_{\beta} g^{i i}-\frac{2 n}{\lambda_{i}^{n} \lambda_{k}} \tilde{R}_{i k i k}-\frac{2 \alpha_{1} n}{\lambda_{i}^{n+1}}\left|\nabla_{\tilde{g}} \phi\right|_{\tilde{g}}^{2} \\
& -\frac{n}{2 \lambda_{i}^{n+1} \lambda_{k} \lambda_{\ell}}\left(\tilde{\nabla}_{k} g_{i \ell}+\tilde{\nabla}_{\ell} g_{i k}-\tilde{\nabla}_{i} g_{\ell k}\right)^{2} .
\end{aligned}
$$

On the other hand, it is not hard to see that

$$
g^{\alpha \beta} \tilde{\nabla}_{\alpha} \tilde{\nabla}_{\beta} u=\frac{n}{\lambda_{i}^{n-1}} g^{\alpha \beta} \tilde{\nabla}_{\alpha} \tilde{\nabla}_{\beta} g^{i i}+n g^{\alpha \beta} \tilde{\nabla}_{\alpha} g^{i j} \tilde{\nabla}_{\beta} g^{i j} \sum_{a=0}^{n-2} \frac{1}{\lambda_{i}^{n-2-a} \lambda_{j}^{a}},
$$

and then

$$
\begin{aligned}
\partial_{t} u= & g^{\alpha \beta} \tilde{\nabla}_{\alpha} \tilde{\nabla}_{\beta} u-\frac{n}{\lambda_{k}} \sum_{a=0}^{n-2} \frac{1}{\lambda_{i}^{n-2-a} \lambda_{j}^{a}}\left(\tilde{\nabla}_{k} g_{i j}\right)^{2}-\frac{2 n}{\lambda_{i}^{n} \lambda_{k}} \tilde{R}_{i k i k} \\
& -\frac{2 \alpha_{1} n}{\lambda_{i}^{n+1}}\left|\nabla_{\tilde{g}} \phi\right|_{\tilde{g}}^{2}-\frac{n}{2 \lambda_{i}^{n+1} \lambda_{k} \lambda_{\ell}}\left(\tilde{\nabla}_{k} g_{i \ell}+\tilde{\nabla}_{\ell} g_{i k}-\tilde{\nabla}_{i} g_{\ell k}\right)^{2} .
\end{aligned}
$$

Since $\left|\operatorname{Rm}_{\tilde{g}}\right|_{\tilde{g}} \leq \sqrt{k_{0}}$, it follows from $(3.9)$ that

$$
\partial_{t} u \leq g^{\alpha \beta} \tilde{\nabla}_{\alpha} \tilde{\nabla}_{\beta} u+2 n \sqrt{k_{0}}\left(\sum_{j=1}^{m} \frac{1}{\lambda_{j}}\right) u .
$$

According to Hölder's inequality

$$
\sum_{j=1}^{m} \frac{1}{\lambda_{j}} \leq\left(\sum_{j=1}^{m} \frac{1}{\lambda_{j}^{n}}\right)^{1 / n}\left(\sum_{j=1}^{m} 1^{n^{\prime}}\right)^{1 / n^{\prime}}=m u^{1 / n}, \quad \frac{1}{n}+\frac{1}{n^{\prime}}=1 ;
$$

therefore $\partial_{t} u \leq g^{\alpha \beta} \tilde{\nabla}_{\alpha} \tilde{\nabla}_{\beta} u+2 n m \sqrt{k_{0}} u^{1+\frac{1}{n}}$. 
As showed in [19, 26], the lower bound of $g_{i j}$ now directly follows from Lemma 3.2

Lemma 3.3. If $\left|\operatorname{Rm}_{\tilde{g}}\right|_{\tilde{g}}^{2} \leq k_{0}$, then, for any $\delta \in(0,1)$, we have

$$
g(t) \geq(1-\delta) \tilde{g}
$$

on $D \times\left[0, T_{-}\left(\delta, m, k_{0}\right)\right]$, where $T_{-}\left(\delta, m, k_{0}\right):=\frac{1}{2 \sqrt{k_{0}}}\left(\frac{1}{m}\right)^{1+1 / n}\left[1-\left(\frac{1}{2}\right)^{1 / n}\right], n$ is a positive integer, and

$$
\frac{\ln (2 m)}{\ln (1 /(1-\delta))} \leq n<\frac{\ln (2 m)}{\ln (1 /(1-\delta))}+1
$$

Since we consider the regular $\left(\alpha_{1}, 0, \beta_{1}, \beta_{2}\right)$-flow, we conclude from Corollary 2.10 that $\left|\nabla_{\hat{g}(t)} \hat{\phi}(t)\right|_{\hat{g}(t)}^{2} \leq C$, where $C$ is a positive constant depending only on $\alpha_{1}, \beta_{2}$, and $\left|\nabla_{\tilde{g}} \tilde{\phi}\right|_{\tilde{g}}^{2}$. Following the arguments in [19, 26], we have an upper bound of $g_{i j}$.

Lemma 3.4. If $|\widetilde{\mathrm{Rm}}|_{\tilde{g}}^{2} \leq k_{0}$ and $|\tilde{\nabla} \tilde{\phi}|_{\tilde{g}}^{2} \leq k_{1}$, then, for any $\theta>0$, we have

$$
g(t) \leq(1+\theta) \tilde{g}
$$

on $D \times\left[0, T_{+}\left(\theta, m, k_{0}, k_{1}, \alpha_{1}, \beta_{2}\right)\right]$, where $T_{+}\left(\delta, m, k_{0}, k_{1}, \alpha_{1}, \beta_{2}\right)$ is a positive constant depending only on $\theta, m, k_{0}, k_{1}, \alpha_{1}$, and $\beta_{2}$.

From Lemma 3.3 and Lemma 3.4 we have

Theorem 3.5. Suppose that $|\widetilde{\mathrm{Rm}}|_{\tilde{g}}^{2} \leq k_{0}$ and $|\tilde{\nabla} \tilde{\phi}|_{\tilde{g}}^{2} \leq k_{1}$ on $M$. If $(g(t), \phi(t))$ is a solution of (3.2), then, for any $\epsilon \in(0,1)$, we have

$$
(1-\epsilon) \tilde{g} \leq g(t) \leq(1+\epsilon) \tilde{g}
$$

on $\bar{D} \times\left[0, T\left(\epsilon, m, k_{0}, k_{1}, \alpha_{1}, \beta_{2}\right)\right]$, where $T\left(\epsilon, m, k_{0}, k_{1}, \alpha_{1}, \beta_{2}\right)$ is a positive constant depending only on $\epsilon, m, k_{0}, k_{1}, \alpha_{1}, \beta_{2}$.

3.2. Existence of the De Turck flow. We establish the short time existence of the De Turck flow $(3.2)$ on the whole manifold $M$. Fix a point $x_{0} \in M$ and let $B_{\tilde{g}}\left(x_{0}, r\right)$ be the metric ball of radius $r$ centered at $x_{0}$ with respect to the metric $\tilde{g}$.

Lemma 3.6. Given positive constants $r, \delta, T$. Suppose that $(g(t), \phi(t))$ is a solution of (3.2) on $B_{\tilde{g}}\left(x_{0}, r+\delta\right) \times[0, T]$, that is,

$$
\begin{aligned}
\partial_{t} g_{i j} & =-2 R_{i j}+2 \alpha_{1} \nabla_{i} \phi \nabla_{j} \phi+\nabla_{i} V_{j}+\nabla_{j} V_{i}, \quad \text { in } B_{\tilde{g}}\left(x_{0}, r+\delta\right) \times[0, T], \\
\partial_{t} \phi & =\Delta \phi+\beta_{1}|\nabla \phi|_{g}^{2}+\beta_{2} \phi+\langle V, \nabla \phi\rangle_{g}, \quad \text { in } B_{\tilde{g}}\left(x_{0}, r+\delta\right) \times[0, T], \\
\left(g_{i j}, \phi\right) & =\left(\tilde{g}_{i j}, \tilde{\phi}\right), \quad \text { on } D_{T},
\end{aligned}
$$

and $|\widetilde{\mathrm{Rm}}|_{\tilde{g}}^{2} \leq k_{0},|\tilde{\nabla} \tilde{\phi}|_{\tilde{g}}^{2} \leq k_{1}$ on $M$. If

$$
\left(1-\frac{1}{80000\left(1+\alpha_{1}^{2}+\beta_{1}^{2}\right) m^{10}}\right) \tilde{g} \leq g(t) \leq\left(1+\frac{1}{80000\left(1+\alpha_{1}^{2}+\beta_{1}^{2}\right) m^{10}}\right) \tilde{g}
$$

on $B_{\tilde{g}}\left(x_{0}, r+\delta\right) \times[0, T]$, then there exists a positive constant $C=C\left(m, r, \delta, T, \tilde{g}, k_{1}\right)$ depending only on $m, r, \delta, T, \tilde{g}$, and $k_{1}$, such that

$$
|\tilde{\nabla} g|_{\tilde{g}}^{2} \leq C, \quad|\tilde{\nabla} \phi|_{\tilde{g}}^{2} \leq C
$$

on $B_{\tilde{g}}\left(x_{0}, r+\frac{\delta}{2}\right) \times[0, T]$. 
Proof. Using the $*$-notion, we can write 2.21) as

$$
\partial_{t} g_{i j}=g^{\alpha \beta} \tilde{\nabla}_{\alpha} \tilde{\nabla}_{\beta} g_{i j}+g^{-1} * g * \widetilde{\mathrm{Rm}}+g^{-1} * g^{-1} * \tilde{\nabla} g * \tilde{\nabla} g+2 \alpha_{1} \tilde{\nabla}_{i} \phi \tilde{\nabla}_{j} \phi .
$$

Then

$$
\begin{aligned}
\partial_{t} \tilde{\nabla} g_{i j}= & g^{\alpha \beta}\left(\nabla \tilde{\nabla}_{\alpha} \tilde{\nabla}_{\beta} g_{i j}\right)+g^{-1} * g^{-1} * \tilde{\nabla} g * \tilde{\nabla} \tilde{\nabla} g+2 \alpha_{1} \tilde{\nabla} i \phi \tilde{\nabla} \tilde{\nabla}_{j} \phi \\
& +g^{-1} * g^{-1} * \tilde{\nabla} g * g * \widetilde{\mathrm{Rm}}+g^{-1} * \tilde{\nabla} g * \widetilde{\mathrm{Rm}} \\
& +g^{-1} * g * \tilde{\nabla} \widetilde{\mathrm{Rm}}+g^{-1} * g^{-1} * g^{-1} * \tilde{\nabla} g * \tilde{\nabla} g * \tilde{\nabla} g
\end{aligned}
$$

since

$$
\begin{aligned}
\tilde{\nabla} \tilde{\nabla}_{\alpha} \tilde{\nabla}_{\beta} g_{i j} & =\tilde{\nabla}_{\alpha} \tilde{\nabla} \tilde{\nabla}_{\beta} g_{i j}+\widetilde{\mathrm{Rm}} * \tilde{\nabla} g \\
& =\tilde{\nabla}_{\alpha}\left(\tilde{\nabla}_{\beta} \tilde{\nabla} g_{i j}+\widetilde{\mathrm{Rm}} * g\right)+\widetilde{\mathrm{Rm}} * \tilde{\nabla} g \\
& =\tilde{\nabla}_{\alpha} \tilde{\nabla}_{\beta} \tilde{\nabla} g_{i j}+g * \tilde{\nabla} \widetilde{\mathrm{Rm}}+\tilde{\nabla} g * \widetilde{\mathrm{Rm}}
\end{aligned}
$$

we conclude from 3.15 that

$$
\begin{aligned}
\partial_{t} \tilde{\nabla} g_{i j}= & g^{\alpha \beta}\left(\tilde{\nabla}_{\alpha} \tilde{\nabla}_{\beta} \tilde{\nabla} g_{i j}\right)+g^{-1} * g^{-1} * \tilde{\nabla} g * \tilde{\nabla} \tilde{\nabla} g+2 \alpha_{1} \tilde{\nabla}_{i} \phi \tilde{\nabla} \tilde{\nabla}_{j} \phi \\
& +g^{-1} * g^{-1} * \tilde{\nabla} g * g * \widetilde{\mathrm{Rm}}+g^{-1} * \tilde{\nabla} g * \widetilde{\mathrm{Rm}} \\
& +g^{-1} * g * \tilde{\nabla} \widetilde{\mathrm{Rm}}+g^{-1} * g^{-1} * g^{-1} * \tilde{\nabla} g * \tilde{\nabla} g * \tilde{\nabla} g .
\end{aligned}
$$

It follows from 3.16 that

$$
\begin{aligned}
\partial_{t}|\tilde{\nabla} g|_{\tilde{g}}^{2}= & 2 \tilde{g}^{\alpha \beta} \tilde{g}^{i k} \tilde{g}^{j \ell} \tilde{\nabla}_{\beta} g_{k \ell} \cdot \partial_{t} \tilde{\nabla}_{\alpha} g_{i j} \\
= & g^{\alpha \beta} \tilde{\nabla}_{\alpha} \tilde{\nabla}_{\beta}|\tilde{\nabla} g|_{\tilde{g}}^{2}-2 g^{\alpha \beta}\langle\tilde{\nabla} \alpha \tilde{\nabla} g, \tilde{\nabla} \beta \tilde{\nabla} g\rangle_{\tilde{g}}+\tilde{\nabla} g * \tilde{\nabla} \phi * \tilde{\nabla} \tilde{\nabla} \phi \\
& +\widetilde{\operatorname{Rm}} * g^{-1} * g^{-1} * g * \tilde{\nabla} g * \tilde{\nabla} g+\widetilde{\operatorname{Rm}} * g^{-1} * \tilde{\nabla} g * \tilde{\nabla} g \\
& +g^{-1} * g * \tilde{\nabla} \widetilde{\operatorname{Rm}} * \tilde{\nabla} g+g^{-1} * g^{-1} * \tilde{\nabla} g * \tilde{\nabla} g * \tilde{\nabla} \tilde{\nabla} g \\
& +g^{-1} * g^{-1} * g^{-1} * \tilde{\nabla} g * \tilde{\nabla} g * \tilde{\nabla} g * \tilde{\nabla} g .
\end{aligned}
$$

Since the closure $\overline{B_{\tilde{g}}\left(x_{0}, r+\delta\right)}$ is compact, we have

$$
|\tilde{\nabla} \widetilde{\mathrm{Rm}}|_{\tilde{g}} \lesssim 1
$$

on $\overline{B_{\tilde{g}}\left(x_{0}, r+\delta\right)}$, where $\lesssim$ depends on $r, \delta, \tilde{g}$. From 3.13 we get

$$
\frac{1}{2} \tilde{g} \leq g(t) \leq 2 \tilde{g}
$$

on $B_{\tilde{g}}\left(x_{0}, r+\delta\right) \times[0, T]$. According to $(3.18)$ and $(3.19)$, we arrive at

$$
\begin{aligned}
\widetilde{\mathrm{Rm}} * g^{-1} * g^{-1} * \tilde{\nabla} g * \tilde{\nabla} g & \lesssim|\tilde{\nabla} g|_{\tilde{g}}^{2}, \\
\widetilde{\mathrm{Rm}} * g^{-1} * \tilde{\nabla} g * \tilde{\nabla} g & \lesssim|\tilde{\nabla} g|_{\tilde{g}}^{2}, \\
\tilde{\nabla} \widetilde{\mathrm{Rm}} * g^{-1} * g * \tilde{\nabla} g & \lesssim|\tilde{\nabla} g|_{\tilde{g}},
\end{aligned}
$$

where $\lesssim$ depends on $m, r, \delta, \tilde{g}$. From the explicit formulas we can see

$$
\begin{aligned}
\tilde{\nabla} g * \tilde{\nabla} \phi * \tilde{\nabla} \tilde{\nabla} \phi & =4 \alpha_{1} \tilde{g}^{\alpha \beta} \tilde{g}^{i k} \tilde{g}^{j \ell} \tilde{\nabla}_{\beta} g_{k \ell} \tilde{\nabla}_{i} \phi \tilde{\nabla}_{\alpha} \tilde{\nabla}_{j} \phi \\
& =4 \alpha_{1} \sum_{1 \leq i, j, \alpha \leq m} \tilde{\nabla}_{\alpha} g_{i j} \tilde{\nabla}_{i} \phi \tilde{\nabla}_{\alpha} \tilde{\nabla}_{j} \phi \\
& \leq 4 \alpha_{1} m^{3}|\tilde{\nabla} g| \tilde{g}|\tilde{\nabla} \phi|_{\tilde{g}}|\tilde{\nabla} \tilde{\nabla} \phi|_{\tilde{g}}
\end{aligned}
$$


where we used a normal coordinate system of $\tilde{g}$. Similarly,

$$
\begin{aligned}
& g^{-1} * g^{-1} * \tilde{\nabla} g * \tilde{\nabla} g * \tilde{\nabla} \tilde{\nabla} g \\
& =\tilde{g}^{\gamma \delta} g^{i k} \tilde{g}^{j \ell} g^{\alpha \beta} g^{p q} \tilde{\nabla}_{\delta} g_{k \ell}\left(\tilde{\nabla}_{\gamma} \tilde{\nabla}_{i} g_{p \alpha} \tilde{\nabla}_{j} g_{q \beta}+\tilde{\nabla}_{i} g_{p \alpha} \tilde{\nabla}_{\gamma} \tilde{\nabla}_{j} g_{q \beta}+2 \tilde{\nabla}_{\gamma} \tilde{\nabla}_{\alpha} g_{j p} \tilde{\nabla}_{q} g_{i \beta}\right. \\
& +2 \tilde{\nabla}_{\alpha} g_{j p} \tilde{\nabla}_{\gamma} \tilde{\nabla}_{q} g_{i \beta}-2 \tilde{\nabla}_{\gamma} \tilde{\nabla}_{\alpha} g_{j p} \tilde{\nabla}_{\beta} g_{i q}-2 \tilde{\nabla}_{\alpha} g_{j p} \tilde{\nabla}_{\gamma} \tilde{\nabla}_{\beta} g_{i q}-2 \tilde{\nabla}_{\gamma} \tilde{\nabla}_{j} g_{p \alpha} \tilde{\nabla}_{\beta} g_{i q} \\
& \left.-2 \tilde{\nabla}_{j} g_{p \alpha} \tilde{\nabla}_{\gamma} \tilde{\nabla}_{\beta} g_{i q}-2 \tilde{\nabla}_{\gamma} \tilde{\nabla}_{i} g_{p \alpha} \tilde{\nabla}_{\beta} g_{j q}-2 \tilde{\nabla}_{i} g_{p \alpha} \tilde{\nabla}_{\gamma} \tilde{\nabla}_{\beta} g_{j q}\right) \\
& =\sum_{1 \leq i, j, \alpha, \gamma, p \leq m} g^{\alpha \alpha} g^{p p} \tilde{\nabla}_{\gamma} g_{i j}\left(\tilde{\nabla}_{\gamma} \tilde{\nabla}_{i} g_{p \alpha} \tilde{\nabla}_{j} g_{p \alpha}+\tilde{\nabla}_{i} g_{p \alpha} \tilde{\nabla}_{j \gamma} g_{p \alpha}\right. \\
& +2 \tilde{\nabla}_{\gamma} \tilde{\nabla}_{\alpha} g_{j p} \tilde{\nabla}_{p} g_{i \alpha}+2 \tilde{\nabla}_{\alpha} g_{j p} \tilde{\nabla}_{\gamma} \tilde{\nabla}_{p} g_{i \alpha}-2 \tilde{\nabla}_{\gamma} \tilde{\nabla}_{\alpha} g_{j p} \tilde{\nabla}_{\alpha} g_{i p} \\
& -2 \tilde{\nabla}_{\alpha} g_{j p} \tilde{\nabla}_{\gamma} \tilde{\nabla}_{\alpha} g_{i p}-2 \tilde{\nabla}_{\gamma} \tilde{\nabla}_{j} g_{p \alpha} \tilde{\nabla}_{\alpha} g_{i p}-2 \tilde{\nabla}_{j} g_{p \alpha} \tilde{\nabla}_{\gamma} \tilde{\nabla}_{\alpha} g_{i p} \\
& \left.-2 \tilde{\nabla}_{\gamma} \tilde{\nabla}_{i} g_{p \alpha} \tilde{\nabla}_{\alpha} g_{j p}-2 \tilde{\nabla}_{i} g_{p \alpha} \tilde{\nabla}_{\gamma} \tilde{\nabla}_{\alpha} g_{j p}\right) \\
& \leq 4 m^{5}(8 \times 2+2)|\tilde{\nabla} g|_{\tilde{g}}^{2}|\tilde{\nabla} \tilde{\nabla} g|_{\tilde{g}}=72 m^{5}|\tilde{\nabla} g|_{\tilde{g}}^{2}|\tilde{\nabla} \tilde{\nabla} g|_{\tilde{g}}
\end{aligned}
$$

and

$$
\begin{aligned}
& g^{-1} * g^{-1} * g^{-1} * \tilde{\nabla} g * \tilde{\nabla} g * \tilde{\nabla} g * \tilde{\nabla} g \\
= & \tilde{g}^{\gamma \delta} \tilde{g}^{i k} \tilde{g}^{j \ell} \tilde{\nabla}_{\delta} g_{k \ell}\left(\tilde{\nabla}_{\gamma} g^{\alpha \beta} g^{p q}+g^{\alpha \beta} \tilde{\nabla}_{\gamma} g^{p q}\right)\left(\tilde{\nabla}_{i} g_{p \alpha} \tilde{\nabla}_{j} g_{q \beta}+2 \tilde{\nabla}_{\alpha} g_{j p} \tilde{\nabla}_{q} g_{i \beta}\right. \\
& \left.-2 \tilde{\nabla}_{\alpha} g_{j p} \tilde{\nabla}_{\beta} g_{i q}-2 \tilde{\nabla}_{j} g_{p \alpha} \tilde{\nabla}_{\beta} g_{i q}-2 \tilde{\nabla}_{i} g_{p \alpha} \tilde{\nabla}_{\beta} g_{j q}\right) \\
\leq & 2^{3} n^{6}(4 \times 2+1) \times 2|\tilde{\nabla} g|_{\tilde{g}}^{4}=144 m^{6}|\tilde{\nabla} g|_{\tilde{g}}^{4} .
\end{aligned}
$$

Thus

$$
\begin{aligned}
\tilde{\nabla} g * \tilde{\nabla} \phi * \tilde{\nabla} \tilde{\nabla} \phi & \leq 4 \alpha_{1} m^{3}|\tilde{\nabla} g|_{\tilde{g}}|\tilde{\nabla} \phi| \tilde{g}|\tilde{\nabla} \tilde{\nabla} \phi|_{\tilde{g}}, \\
g^{-1} * g^{-1} * \tilde{\nabla} g * \tilde{\nabla} g * \tilde{\nabla} \tilde{\nabla} g & \leq 72 m^{5}|\tilde{\nabla} g|_{\tilde{g}}^{2}|\tilde{\nabla} \tilde{\nabla} g|_{\tilde{g}}, \\
g^{-1} * g^{-1} * g^{-1} * \tilde{\nabla} g * \tilde{\nabla} g * \tilde{\nabla} g * \tilde{\nabla} g & \leq 144 m^{6}|\tilde{\nabla} g|_{\tilde{g}}^{4} .
\end{aligned}
$$

Furthermore, using $(3.19)$, we get

$$
g^{\alpha \beta}\left\langle\tilde{\nabla}_{\alpha} \tilde{\nabla} g, \tilde{\nabla}_{\beta} \tilde{\nabla} g\right\rangle_{\tilde{g}}=g^{\alpha \beta} \tilde{g}^{i k} \tilde{g}^{j \ell} \tilde{g}^{\gamma \delta} \tilde{\nabla}_{\alpha} \tilde{\nabla}_{\gamma} g_{i j} \tilde{\nabla}_{\beta} \tilde{\nabla}_{\delta} g_{k \ell} \geq \frac{1}{2}|\tilde{\nabla} \tilde{\nabla} g|_{\tilde{g}}^{2} .
$$

Substituting (3.20), (3.21), and (3.22) into (3.16) implies

$\partial_{t}|\tilde{\nabla} g|_{\tilde{g}}^{2} \leq g^{\alpha \beta} \tilde{\nabla}_{\alpha} \tilde{\nabla}_{\beta}|\tilde{\nabla} g|_{\tilde{g}}^{2}-\left|\tilde{\nabla}^{2} g\right|_{\tilde{g}}^{2}+C_{1}|\tilde{\nabla} g|_{\tilde{g}}^{2}+C_{1}|\tilde{\nabla} g|_{\tilde{g}}$

for some positive constant $C_{1}$ depending only on $m, r, \delta, \tilde{g}$.

Using 2.22, we have

$$
\begin{aligned}
\partial_{t} \tilde{\nabla}_{k} \phi & =\tilde{\nabla}_{k}\left(g^{i j} \tilde{\nabla}_{i} \tilde{\nabla}_{j} \phi+\beta_{1}|\tilde{\nabla} \phi|_{g}^{2}+\beta_{2} \phi\right) \\
& =\tilde{\nabla}_{k} g^{i j} \tilde{\nabla}_{i} \tilde{\nabla}_{j} \phi+g^{i j} \tilde{\nabla}_{k} \tilde{\nabla}_{i} \tilde{\nabla}_{j} \phi+\beta_{1} \tilde{\nabla}_{k}\left(g^{i j} \tilde{\nabla}_{i} \phi \tilde{\nabla}_{j} \phi\right)+\beta_{2} \tilde{\nabla}_{k} \phi ;
\end{aligned}
$$

from the Ricci identity,

$$
\tilde{\nabla}_{k} \tilde{\nabla}_{i} \tilde{\nabla}_{j} \phi=\tilde{\nabla}_{i} \tilde{\nabla}_{k} \tilde{\nabla}_{j} \phi-\tilde{R}_{k i j p} \tilde{\nabla}^{\ell} \phi=\tilde{\nabla}_{i} \tilde{\nabla}_{j} \tilde{\nabla}_{k} \phi-\tilde{R}_{k i j p} \tilde{\nabla}^{p} \phi
$$


we obtain

$$
\begin{aligned}
\partial_{t} \tilde{\nabla}_{k} \phi= & g^{i j} \tilde{\nabla}_{i} \tilde{\nabla}_{j}\left(\tilde{\nabla}_{k} \phi\right)-g^{i j} \tilde{R}_{k i j p} \tilde{\nabla}^{p} \phi+\tilde{\nabla}_{k} g^{i j} \tilde{\nabla}_{i} \tilde{\nabla}_{j} \phi \\
& +\beta_{1} \tilde{\nabla}_{k} g^{i j} \tilde{\nabla}_{i} \phi \tilde{\nabla}_{j} \phi+2 \beta_{1} g^{i j} \tilde{\nabla}_{j} \phi \tilde{\nabla}_{i} \tilde{\nabla}_{k} \phi+\beta_{2} \tilde{\nabla}_{k} \phi
\end{aligned}
$$

The evolution equation (3.24) gives us the following equation

$$
\begin{aligned}
\partial_{t}|\tilde{\nabla} \phi|_{\tilde{g}}^{2}= & \partial_{t}\left(\tilde{g}^{k \ell} \tilde{\nabla}_{k} \phi \tilde{\nabla}_{\ell} \phi\right)=2 \tilde{g}^{k \ell} \tilde{\nabla}_{\ell} \phi\left(\partial_{t} \tilde{\nabla}_{k} \phi\right) \\
= & g^{i j}\left(\tilde{\nabla}_{i} \tilde{\nabla}_{j}\left(\tilde{\nabla}_{k} \phi\right) \cdot 2 \tilde{g}^{k \ell} \tilde{\nabla}_{\ell} \phi\right)-2 g^{i j} \tilde{g}^{k \ell} \tilde{R}_{k i j p} \tilde{\nabla}_{\ell} \phi \tilde{\nabla}^{p} \phi \\
& +2 \tilde{g}^{k \ell} \tilde{\nabla}_{k} g^{i j} \tilde{\nabla}_{\ell} \phi \tilde{\nabla}_{i} \tilde{\nabla}_{j} \phi+2 \beta_{1} \tilde{g}^{k \ell} \tilde{\nabla}_{k} g^{i j} \tilde{\nabla}_{i} \phi \tilde{\nabla}_{j} \phi \tilde{\nabla}_{\ell} \phi \\
& +4 \beta_{1} \tilde{g}^{k \ell} g^{i j} \tilde{\nabla}_{j} \phi \tilde{\nabla}_{\ell} \phi \tilde{\nabla}_{i} \tilde{\nabla}_{k} \phi+2 \beta_{2} \tilde{g}^{k \ell} \tilde{\nabla}_{k} \phi \tilde{\nabla}_{\ell} \phi .
\end{aligned}
$$

The identity

$$
g^{i j} \tilde{\nabla}_{i} \tilde{\nabla}_{j}|\tilde{\nabla} \phi|_{\tilde{g}}^{2}=g^{i j}\left(\tilde{\nabla}^{i} \tilde{\nabla}_{j}\left(\tilde{\nabla}_{k} \phi\right) \cdot 2 \tilde{g}^{k \ell} \tilde{\nabla}_{\ell} \phi\right)+2 g^{i j} \tilde{g}^{k \ell} \tilde{\nabla}_{i} \tilde{\nabla}_{k} \phi \tilde{\nabla}_{j} \tilde{\nabla}_{\ell} \phi
$$

together with 3.18, implies that

$$
g^{i j}\left(\tilde{\nabla}_{i} \tilde{\nabla}_{j}\left(\tilde{\nabla}_{k} \phi\right) \cdot 2 \tilde{g}^{k \ell} \tilde{\nabla}_{\ell} \phi\right) \leq g^{i j} \tilde{\nabla}_{i} \tilde{\nabla}_{j}|\tilde{\nabla} \phi|_{\tilde{g}}^{2}-\left|\tilde{\nabla}^{2} \phi\right|_{\tilde{g}}^{2} .
$$

Using 3.18 again, we find that

$$
\begin{aligned}
-2 g^{i j} \tilde{g}^{k \ell} \tilde{R}_{k i j p} \tilde{\nabla}_{\ell} \phi \tilde{\nabla}^{p} \phi & =-2 g^{i j} \tilde{g}^{k \ell} \tilde{g}^{p q} \tilde{R}_{k i j p} \tilde{\nabla}_{\ell} \phi \tilde{\nabla}_{q} \phi \\
& \lesssim|\tilde{\nabla} \phi|_{\tilde{g}}^{2}, \\
2 \tilde{g}^{k \ell} \tilde{\nabla}_{k} g^{i j} \tilde{\nabla}_{\ell} \phi \tilde{\nabla}_{i} \tilde{\nabla}_{j} \phi & =-2 \tilde{g}^{k \ell} g^{i p} g^{q j} \tilde{\nabla}_{k} g_{p q} \tilde{\nabla}_{\ell} \phi \tilde{\nabla}_{i} \tilde{\nabla}_{j} \phi \\
& \leq 8 m^{3}|\tilde{\nabla} g|_{\tilde{g}}|\tilde{\nabla} \phi|_{\tilde{g}}\left|\tilde{\nabla}^{2} \phi\right|_{\tilde{g}}, \\
\tilde{g}^{k \ell} \tilde{\nabla}_{k} g^{i j} \tilde{\nabla}_{i} \phi \tilde{\nabla}_{j} \phi \tilde{\nabla}_{\ell} \phi & =-\tilde{g}^{k \ell} g^{i p} g^{j q} \tilde{\nabla}_{k} g_{p q} \tilde{\nabla}_{i} \phi \tilde{\nabla}_{j} \phi \tilde{\nabla}_{\ell} \phi \\
& \leq 4 m^{3}|\tilde{\nabla} g|_{\tilde{g}}|\tilde{\nabla} \phi|_{\tilde{g}}^{3}, \\
\tilde{g}^{k \ell} g^{i j} \tilde{\nabla}_{j} \phi \tilde{\nabla}_{\ell} \phi \tilde{\nabla}_{i} \tilde{\nabla}_{k} \phi & \leq 4 m^{2}|\tilde{\nabla} \phi|_{\tilde{g}}^{2}\left|\tilde{\nabla}^{2} \phi\right|_{\tilde{g}},
\end{aligned}
$$

where $\lesssim$ depends on $m, r, \delta, \tilde{g}$. Substituting those estimates and (3.26) into (3.25), we arrive at

$$
\begin{aligned}
\partial_{t}|\tilde{\nabla} \phi|_{\tilde{g}}^{2} \leq & g^{i j} \tilde{\nabla}_{i} \tilde{\nabla}_{j}|\tilde{\nabla} \phi|_{\tilde{g}}^{2}-\left|\tilde{\nabla}^{2} \phi\right|_{\tilde{g}}^{2}+C_{2}|\tilde{\nabla} \phi|_{\tilde{g}}^{2}+8 m^{3}|\tilde{\nabla} g|_{\tilde{g}}|\tilde{\nabla} \phi|_{\tilde{g}}\left|\tilde{\nabla}^{2} \phi\right|_{\tilde{g}} \\
& +8 \beta_{1} m^{3}|\tilde{\nabla} g|_{\tilde{g}}|\tilde{\nabla} \phi|_{\tilde{g}}^{3}+16 \beta_{1} m^{2}|\tilde{\nabla} \phi|_{\tilde{g}}^{2}\left|\tilde{\nabla}^{2} \phi\right|_{\tilde{g}}+2 \beta_{2}|\tilde{\nabla} \phi|_{\tilde{g}}^{2}
\end{aligned}
$$

As in [19, we consider the vector-valued tensor field

$$
\boldsymbol{\Theta}(t):=(g(t), \phi(t))
$$

and define

$$
\tilde{\nabla}^{k} \Theta(t):=\left(\tilde{\nabla}^{k} g(t), \tilde{\nabla}^{k} \phi(t)\right), \quad\left|\tilde{\nabla}^{k} \Theta(t)\right|_{\tilde{g}}^{2}:=\left|\tilde{\nabla}^{k} g(t)\right|_{\tilde{g}}^{2}+\left|\tilde{\nabla}^{k} \phi(t)\right|_{\tilde{g}}^{2} .
$$

From 3.23 and 3.27, we obtain

$$
\partial_{t}|\tilde{\nabla} \Theta|_{\tilde{g}}^{2} \leq g^{\alpha \beta} \tilde{\nabla}_{\alpha} \tilde{\nabla}_{\beta}|\tilde{\nabla} \boldsymbol{\Theta}|_{\tilde{g}}^{2}-\left|\tilde{\nabla}^{2} \Theta\right|_{\tilde{g}}^{2}+C_{3}|\tilde{\nabla} \Theta|_{\tilde{g}}^{2}+C_{3}|\tilde{\nabla} \Theta|_{\tilde{g}}
$$

$$
+\left.\left(80+4 \alpha_{1}+16 \beta_{1}\right) m^{5}|\tilde{\nabla} \Theta|\right|_{\tilde{g}} ^{2}\left|\tilde{\nabla}^{2} \Theta\right|_{\tilde{g}}+\left(144+8 \beta_{1}\right) m^{6}|\tilde{\nabla} \Theta|_{\tilde{g}}^{4}
$$

where $C_{3}$ is positive constant depending on $m, r, \delta, \tilde{g}$ and $\beta_{2}$. The inequality (3.29) is similar to the equation (11) in page 247 of [26] and the equation (3.28) in page 36 of [19, so that the proof is essentially without change anything. However, for our flow, we want to find the positive constant $\epsilon>0$ with $(1-\epsilon) \tilde{g} \leq g \leq(1+\epsilon) \tilde{g}$ on 
$B_{\tilde{g}}\left(x_{0}, r+\delta\right) \times[0, T]$ such that both functions $|\tilde{\nabla} g|_{\tilde{g}}^{2}$ and $|\tilde{\nabla} \phi|_{\tilde{g}}^{2}$ are bounded from above. The mentioned positive constant $\epsilon$ depends on $m, \alpha_{1}, \beta_{1}$, and $\beta_{2}$; the aim of the following computations is to find an explicit formula for $\epsilon$. We shall follow Shi's idea but do more slight work on calculus, in particular on the positive uniform constants we are going to obtain.

By the inequality

$$
a b \leq \epsilon a^{2}+\frac{1}{4 \epsilon} b^{2}, \quad a, b \in \mathbf{R}, \quad \epsilon>0,
$$

we have

$$
\left(80+4 \alpha_{1}+16 \beta_{1}\right) m^{5}|\tilde{\nabla} \Theta|_{\tilde{g}}^{2}\left|\tilde{\nabla}^{2} \boldsymbol{\Theta}\right|_{\tilde{g}} \leq \frac{1}{2}\left|\tilde{\nabla}^{2} \boldsymbol{\Theta}\right|_{\tilde{g}}^{2}+2\left(40+2 \alpha_{1}+8 \beta_{1}\right)^{2} m^{10}|\tilde{\nabla} \Theta|_{\tilde{g}}^{4}
$$

and

$$
C_{3}|\tilde{\nabla} \Theta|_{\tilde{g}} \leq C_{3} \frac{1+|\tilde{\nabla} \Theta|_{\tilde{g}}^{2}}{2}=\frac{C_{3}}{2}+\frac{C_{3}}{2}|\tilde{\nabla} \Theta|_{\tilde{g}}^{2}
$$

As a consequence of $(3.29)$, we conclude that

$$
\begin{aligned}
\partial_{t}|\tilde{\nabla} \Theta|_{\tilde{g}}^{2} \leq & g^{\alpha \beta} \tilde{\nabla}_{\alpha} \tilde{\nabla}_{\beta}|\tilde{\nabla} \Theta|_{\tilde{g}}^{2}-\frac{1}{2}\left|\tilde{\nabla}^{2} \Theta\right|_{\tilde{g}}^{2}+C_{4}|\tilde{\nabla} \Theta|_{\tilde{g}}^{2}+C_{4}+\left(3344+8 \alpha_{1}^{2}\right. \\
& \left.+320 \alpha_{1}+64 \alpha_{1} \beta_{1}+128 \beta_{1}^{2}+1280 \beta_{1}+8 \beta_{1}\right) m^{10}|\tilde{\nabla} \Theta|_{\tilde{g}}^{4}
\end{aligned}
$$

for some positive constant $C_{4}$ depending only on $m, r, \delta, \tilde{g}$ and $\beta_{2}$.

Given $\epsilon:=\frac{1}{A m^{10}} \leq \frac{1}{2}$, where $A$ is a positive constant depending on $\alpha_{1}, \beta_{1}$ and chosen later. If we choose a normal coordinate system so that

$$
\tilde{g}_{i j}=\delta_{i j}, \quad g_{i j}=\lambda_{i} \delta_{i j},
$$

then we have

$$
1-\epsilon \leq \lambda_{k} \leq 1+\epsilon, \quad \frac{1}{2} \leq \lambda_{k} \leq 2, \quad k=1, \cdots, m .
$$

Define

$$
n:=\frac{1}{\epsilon}, \quad a:=\frac{n}{4}
$$

and

$$
\varphi=\varphi(x, t):=a+\sum_{1 \leq k \leq m} \lambda_{k}^{n}, \quad(x, t) \in B_{\tilde{g}}\left(x_{0}, r+\delta\right) \times[0, T] .
$$

By the formula (16) in page 248 of [26], we can compute

$$
\begin{aligned}
\partial_{t} \varphi= & n \sum_{1 \leq k, \alpha, \beta \leq m} \lambda_{k}^{n-1} g^{\alpha \beta} \tilde{\nabla}_{\alpha} \tilde{\nabla}_{\beta} g_{k k}+2 n \sum_{1 \leq k, \alpha \leq m} \lambda_{k}^{n-1} \frac{\lambda_{k}}{\lambda_{\alpha}} \tilde{R}_{k \alpha k \alpha} \\
& +\frac{n}{2} \sum_{1 \leq k, \alpha, p \leq m} \frac{\lambda_{k}^{n-1}}{\lambda_{\alpha} \lambda_{p}}\left(\tilde{\nabla}_{k} g_{p \alpha} \tilde{\nabla}_{k} g_{p \alpha}+2 \tilde{\nabla}_{\alpha} g_{k p} \tilde{\nabla}_{p} g_{k \alpha}-2 \tilde{\nabla}_{\alpha} g_{k p} \tilde{\nabla}_{\alpha} g_{k p}\right. \\
& \left.-2 \tilde{\nabla}_{k} g_{p \alpha} \tilde{\nabla}_{\alpha} g_{k p}-2 \tilde{\nabla}_{k} g_{p \alpha} \tilde{\nabla}_{\alpha} g_{k p}\right) .
\end{aligned}
$$

Since the second and the third on the right-hand side of 3.35 is bounded from above by $4 n(1+\epsilon)^{n} m^{2} \sqrt{k_{0}}$ and

$$
\frac{n}{2} m^{3}(1+\epsilon)^{n-1} 4(4 \times 2+1)|\tilde{\nabla} g|_{\tilde{g}}^{2}=18 n m^{3}(1+\epsilon)^{n-1}|\tilde{\nabla} g|_{\tilde{g}}^{2},
$$


respectively, it follows that

$$
\partial_{t} \varphi \leq n \sum_{1 \leq k, \alpha, \beta \leq m} \lambda_{k}^{n-1} g^{\alpha \beta} \tilde{\nabla}_{\alpha} \tilde{\nabla}_{\beta} g_{k k}+C_{5}+18 n m^{3}(1+\epsilon)^{n-1}|\tilde{\nabla} g|_{\tilde{g}}^{2}
$$

where $C_{5}$ is a positive constant depending only on $m, A$, and $k_{0}$. On the other hand, from (3.36) and the equation (19) in page 249 of [26], we have

$$
\partial_{t} \varphi \leq g^{\alpha \beta} \tilde{\nabla}_{\alpha} \tilde{\nabla}_{\beta} \varphi+C_{5}+\left[18 m^{3} n(1+\epsilon)^{n-1}-\frac{n(n-1)}{2}(1-\epsilon)^{n-2}\right]|\tilde{\nabla} g|_{\tilde{g}}^{2} .
$$

Instead of the inequalities (20), (21), and (22) in page 249 of [26, we will prove uniform inequalities as follows (recall that $\epsilon:=1 / n$ ):

(a) For any $n \geq 2$ and any $m$, we have

$$
18 m^{3} n(1+\epsilon)^{n-1} \leq \frac{54 m^{3}}{n+1} n^{2} .
$$

(b) For any $n \geq 2$, we have

$$
(1-\epsilon)^{n-2} \geq \frac{1}{4}
$$

(c) For any $n \geq 2$, we have

$$
\frac{n(n-1)}{2}(1-\epsilon)^{n-2} \geq \frac{n^{2}}{8} .
$$

To prove (3.38), we write

$$
18 m^{3} n(1+\epsilon)^{n-1}=18 n m^{3} \frac{n}{n+1}\left(1+\frac{1}{n}\right)^{n}=\frac{18 m^{3} n^{2}}{n+1}\left(1+\frac{1}{n}\right)^{n} ;
$$

since the function $(1+1 / x)^{x}, x>0$, is in increasing in $x$, it follows that

$$
18 m^{3} n(1+\epsilon)^{n-1} \leq \frac{18 m^{3} n^{2}}{n+1} e \leq \frac{54 m^{3}}{n+1} n^{2} .
$$

Since the function $(1-1 / x)^{x}, x \geq 2$, is increasing in $x$, we obtain

$$
(1-\epsilon)^{n-2}=\left(1-\frac{1}{n}\right)^{n-2}=\frac{n^{2}}{(n-1)^{2}}\left(1-\frac{1}{n}\right)^{n} \geq\left(1-\frac{1}{n}\right)^{n} \geq\left(1-\frac{1}{2}\right)^{2}=\frac{1}{4} .
$$

From the proof of $(3.39)$, together with 3.38 , we arrive at

$$
\frac{n(n-1)}{2}(1-\epsilon)^{n-2} \geq \frac{n(n-1)}{2} \frac{n^{2}}{(n-1)^{2}} \frac{1}{4}=\frac{n^{2}}{8} \frac{n}{n-1} \geq \frac{n^{2}}{8} .
$$

Substituting (3.38) and (3.40) into (3.37) implies

$$
\partial_{t} \varphi \leq g^{\alpha \beta} \tilde{\nabla}_{\alpha} \tilde{\nabla}_{\beta} \varphi+C_{5}-\left(\frac{1}{8}-\frac{54 m^{3}}{n+1}\right) n^{2}|\tilde{\nabla} g|_{\tilde{g}}^{2} ;
$$

choosing

$$
n \geq 864 m^{10}>864 m^{3},
$$

we find that

$$
\partial_{t} \varphi \leq g^{\alpha \beta} \tilde{\nabla}_{\alpha} \tilde{\nabla}_{\beta} \varphi+C_{5}-\frac{n^{2}}{16}|\tilde{\nabla} g|_{\tilde{g}}^{2}
$$


As the equation (24) in page 249 of [26, we have

$$
\begin{aligned}
\partial_{t}\left(\varphi|\tilde{\nabla} \Theta|_{\tilde{g}}^{2}\right) \leq & g^{\alpha \beta} \tilde{\nabla}_{\alpha} \tilde{\nabla}_{\beta}\left(\varphi|\tilde{\nabla} \Theta|_{\tilde{g}}^{2}\right)-2 g^{\alpha \beta} \tilde{\nabla}_{\alpha} \varphi \tilde{\nabla}{ }_{\beta}|\tilde{\nabla} \Theta|_{\tilde{g}}^{2}-\frac{1}{2} \varphi\left|\tilde{\nabla}^{2} \Theta\right|_{\tilde{g}}^{2} \\
& +\left(3344+8 \alpha_{1}^{2}+320 \alpha_{1}+64 \alpha_{1} \beta_{1}+128 \beta_{1}^{2}+1280 \beta_{1}+8 \beta_{1}\right) \times \\
& m^{10} \varphi|\tilde{\nabla} \Theta|_{\tilde{g}}^{4}+C_{4} \varphi|\tilde{\nabla} \Theta|_{\tilde{g}}^{2}+C_{4} \varphi+C_{5}|\tilde{\nabla} \Theta|_{\tilde{g}}^{2}-\frac{n^{2}}{16}|\tilde{\nabla} \Theta|_{\tilde{g}}^{2}|\tilde{\nabla} g|_{\tilde{g}}^{2}
\end{aligned}
$$

According to Corollary 2.10 , we get $|\nabla \phi|_{g}^{2} \lesssim 1$ and hence

$$
|\tilde{\nabla} \phi|_{\tilde{g}}^{2}=\tilde{g}^{\alpha \beta} \tilde{\nabla}_{\alpha} \tilde{\nabla}_{\beta} \phi \leq 2|\nabla \phi|_{g}^{2} \lesssim 1
$$

where $\lesssim$ depends on $\alpha_{1}, \beta_{2}$ and $k_{1}$. Consequently, 3.43 can be written as

$$
\begin{aligned}
\partial_{t}\left(\varphi|\tilde{\nabla} \Theta|_{\tilde{g}}^{2}\right) \leq & g^{\alpha \beta} \tilde{\nabla}_{\alpha} \tilde{\nabla}_{\beta}\left(\varphi|\tilde{\nabla} \Theta|_{\tilde{g}}^{2}\right)-2 g^{\alpha \beta} \tilde{\nabla}_{\alpha} \varphi \tilde{\nabla}_{\beta}|\tilde{\nabla} \Theta|_{\tilde{g}}^{2}-\frac{1}{2} \varphi\left|\tilde{\nabla}^{2} \Theta\right|_{\tilde{g}}^{2} \\
& +\left(3344+8 \alpha_{1}^{2}+320 \alpha_{1}+64 \alpha_{1} \beta_{1}+128 \beta_{1}^{2}+1280 \beta_{1}+8 \beta_{1}\right) \times \\
& m^{10} \varphi|\tilde{\nabla} \Theta|_{\tilde{g}}^{4}+C_{4} \varphi|\tilde{\nabla} \Theta|_{\tilde{g}}^{2}+C_{4} \varphi+C_{6}|\tilde{\nabla} \Theta|_{\tilde{g}}^{2}-\frac{n^{2}}{16}|\tilde{\nabla} \Theta|_{\tilde{g}}^{4},
\end{aligned}
$$

where $C_{6}$ is a positive constant depending only on $m, A, k_{0}, n, \alpha_{1}, \beta_{2}$, and $k_{1}$. From 3.32 and 3.34,

$$
a+m(1-\epsilon)^{n} \leq \varphi \leq a+m(1+\epsilon)^{n}
$$

on $B_{\tilde{g}}\left(x_{0}, r+\delta\right) \times[0, T]$, we arrive at (recall from 3.41 that $n=A m^{10}$ with $A \geq 864)$

$$
C m^{10} \varphi \leq C m^{10}\left[\frac{n}{4}+m\left(1+\frac{1}{n}\right)^{n}\right] \leq C m^{10}\left(\frac{n}{4}+3 m\right) \leq C m^{10} \frac{n}{2}=\frac{n^{2}}{2 A / C},
$$

where

$$
C:=3344+8 \alpha_{1}^{2}+320 \alpha_{1}+64 \alpha_{1} \beta_{1}+128 \beta_{1}^{2}+1280 \beta_{1}+8 \beta_{1} .
$$

If we choose

$$
A \geq 16 C,
$$

then

$$
C m^{10} \varphi|\tilde{\nabla} \Theta|_{\tilde{g}}^{4}-\frac{n^{2}}{16}|\tilde{\nabla} \Theta|_{\tilde{g}}^{4} \leq-\frac{n^{2}}{32}|\tilde{\nabla} \Theta|_{\tilde{g}}^{4} .
$$

On the other hand, by the argument in the proof of (28) in page 250 of [26], we have

$$
-2 g^{\alpha \beta} \tilde{\nabla}_{\alpha} \varphi \tilde{\nabla}_{\beta}|\tilde{\nabla} \Theta|_{\tilde{g}}^{2} \leq \frac{\varphi}{2}\left|\tilde{\nabla}^{2} \Theta\right|_{\tilde{g}}^{2}+\frac{288 n^{2} m^{10}}{\varphi}|\tilde{\nabla} \Theta|_{\tilde{g}}^{4} .
$$

Plugging 3.48 and 3.49 into 3.44 implies

$$
\begin{aligned}
\partial_{t}\left(\varphi|\tilde{\nabla} \Theta|_{\tilde{g}}^{2}\right) \leq & g^{\alpha \beta} \tilde{\nabla}_{\alpha} \tilde{\nabla} \beta\left(\varphi|\tilde{\nabla} \Theta|_{\tilde{g}}^{2}\right)+\frac{288 n^{2} m^{10}}{\varphi}|\tilde{\nabla} \Theta|_{\tilde{g}}^{4} \\
& -\frac{n^{2}}{32}|\tilde{\nabla} \Theta|_{\tilde{g}}^{4}+C_{4} \varphi+\left(C_{4}+C_{6}\right) \varphi|\tilde{\nabla} \Theta|_{\tilde{g}}^{2},
\end{aligned}
$$

because $\varphi \geq a=\frac{n}{4} \geq 1$. According to $\varphi \geq a=\frac{n}{4}=\frac{A}{4} m^{10}$, we conclude that

$$
\frac{28 n^{2} m^{10}}{\varphi} \leq 1152 n m^{10}=\frac{n^{2}}{A / 1152} \leq \frac{n^{2}}{64}
$$


where we choose

$$
A \geq 1152 \times 64=73728
$$

Consequently,

$$
\partial_{t}\left(\varphi|\tilde{\nabla} \Theta|_{\tilde{g}}^{2}\right) \leq g^{\alpha \beta} \tilde{\nabla}_{\alpha} \tilde{\nabla}_{\beta}\left(\varphi|\tilde{\nabla} \Theta|_{\tilde{g}}^{2}\right)-\frac{n^{2}}{64}|\tilde{\nabla} \Theta|_{\tilde{g}}^{4}+C_{4} \varphi+\left(C_{4}+C_{6}\right) \varphi|\tilde{\nabla} \Theta|_{\tilde{g}}^{2} .
$$

Using the following inequality

$$
\begin{aligned}
\varphi & \leq a+m(1+\epsilon)^{n}=\frac{n}{4}+m\left(1+\frac{1}{n}\right)^{n} \leq \frac{n}{4}+3 m \\
& \leq \frac{n}{4}+\frac{3 n}{A}=\left(\frac{1}{4}+\frac{3}{A}\right) n \leq \frac{18435}{73728} n \leq 0.26 n
\end{aligned}
$$

by (3.51), we get

$$
\frac{n^{2}}{64}|\tilde{\nabla} \Theta|_{\tilde{g}}^{4}=\frac{n^{2}}{64 \varphi^{2}} \varphi^{2}|\tilde{\nabla} \Theta|_{\tilde{g}}^{2} \geq \frac{n^{2}}{4.3264 n^{2}} \varphi^{2}|\tilde{\nabla} \Theta|_{\tilde{g}}^{4} \geq \frac{1}{5} \varphi^{2}|\tilde{\nabla} g|_{\tilde{g}}^{4} .
$$

Defining

$$
\psi:=\varphi|\tilde{\nabla} \Theta|_{\tilde{g}}^{2},
$$

we obtain from the above the inequality and 3.52 that

$$
\partial_{t} \psi \leq g^{\alpha \beta} \tilde{\nabla}_{\alpha} \tilde{\nabla}_{\beta}-\frac{1}{5} \psi^{2}+\left(C_{4}+C_{6}\right) \psi+C_{4} n \leq g^{\alpha \beta} \tilde{\nabla}_{\alpha} \tilde{\nabla}_{\beta} \psi-\frac{1}{10} \psi^{2}+C_{7}
$$

on $B_{\tilde{g}}\left(x_{0}, r+\delta\right) \times[0, T]$, for some positive constant $C_{7}$ depending only $m, A, k_{0}, n, \alpha_{1}, \beta_{2}$, and $k_{1}$.

Using the cutoff function and going through the argument in [26, we can prove that

$$
|\tilde{\nabla} \Theta|_{\tilde{g}}^{2} \lesssim 1
$$

on $B_{\tilde{g}}\left(x_{0}, r+\frac{\delta}{2}\right) \times[0, T]$, where $\lesssim$ depends on $m, r, \delta, T, \tilde{g}, k_{1}$. Note that that $\left(1-\frac{1}{A m^{10}}\right) \tilde{g} \leq g \leq\left(1+\frac{1}{A m^{10}} \tilde{g}\right.$, where

$$
A \geq \max (73728,16 C) \text {. }
$$

From the definition (3.46), we can estimate

$$
\begin{aligned}
C \leq & 3344+8 \alpha_{1}^{2}+160+160 \alpha_{1}^{2}+32\left(\alpha_{1}^{2}+\beta_{1}^{2}\right) \\
& +64+64 \beta_{1}^{2}+640+640 \beta_{1}^{2}+4+4 \beta_{1}^{2} \\
\leq & 4212+200 \alpha_{1}^{2}+740 \beta_{1}^{2} .
\end{aligned}
$$

Then we may choose $A=80000\left(1+\alpha_{1}^{2}+\beta_{1}^{2}\right)$.

By the same method we can prove the higher order derivatives estimates for $g$.

Lemma 3.7. Under the assumption in Lemma 3.6 where we furthermore assume $|\phi|^{2} \leq k_{1}$, for any nonnegative integer $n$, there exist positive constants $C_{n}=$ $C\left(m, n, r, \delta, T, \tilde{g}, k_{1}\right)$ depending only on $m, n, r, \delta, T, \tilde{g}$, and $k_{1}$, such that

$$
\left|\tilde{\nabla}^{n} g\right|_{\tilde{g}}^{2} \leq C_{n}, \quad\left|\tilde{\nabla}^{n} \phi\right|_{\tilde{g}}^{2} \leq C_{n}
$$

on $B_{\tilde{g}}\left(x_{0}, r+\frac{\delta}{n+1}\right) \times[0, T]$. 
Proof. We prove this lemma by induction on $n$. If $n=0$, using (3.19) we have

$$
|g|_{\tilde{g}}^{2}=\tilde{g}^{i k} \tilde{g}^{j \ell} g_{i j} g_{k \ell} \leq 4 m
$$

on $B_{\tilde{g}}\left(x_{0}, r+\delta\right) \times[0, T]$. Since $|\tilde{\phi}|_{\tilde{g}}^{2} \leq k_{1}$, it follows from 3.14 that $|\phi|_{\tilde{g}}^{2} \lesssim 1$ on $B_{\tilde{g}}\left(x_{0}, r+\frac{\delta}{2}\right) \times[0, T]$, where $\lesssim$ depends only on $m, r, \delta, T, \tilde{g}, k_{1}$ and is independent on $x_{0}$. Now we consider the annulus $B_{\tilde{g}}\left(x_{0}, r+\delta\right) \backslash B_{\tilde{g}}\left(x_{0}, r+\frac{\delta}{2}\right)$. For any $x$ in this annulus, we can find a small ball $B_{\tilde{g}}\left(x, \delta^{\prime}\right) \subset B_{\tilde{g}}\left(x_{0}, r+\delta\right) \backslash B_{\tilde{g}}\left(x_{0}, r+\frac{\delta}{2}\right)$. Using 3.14 again, we have $|\phi|_{\tilde{g}}^{2} \lesssim 1$ on $B_{\tilde{g}}\left(x, \frac{\delta^{\prime}}{2}\right) \times[0, T]$, where $\lesssim$ depends only on $m, r, \delta, T, \tilde{g}, k_{1}$ and is independent on $x$. In particular, $|\phi|_{\tilde{g}}^{2}(x) \lesssim 1$ on $[0, T]$. Hence $|\phi|_{\tilde{g}}^{2} \lesssim 1$ on $B_{\tilde{g}}\left(x_{0}, r+\delta\right) \times[0, T]$, where $\lesssim$ depends only on $m, r, \delta, T, \tilde{g}, k_{1}$ and is independent on $x_{0}$.

If $n=1$, the estimate 3.55 was proved in Lemma 3.6 . We now suppose that $n \geq 2$ and

$$
\left|\tilde{\nabla}^{k} g\right|_{\tilde{g}}^{2} \leq C_{k}, \quad\left|\tilde{\nabla}^{k} \phi\right|_{\tilde{g}}^{2} \leq C_{k}, \quad k=0,1, \cdots, n-1,
$$

on $B_{\tilde{g}}\left(x_{0}, r+\frac{\delta}{k+1}\right) \times[0, T]$. According to (3.39) in [19], we have

$$
\begin{aligned}
\partial_{t} \tilde{\nabla}^{n} g= & g^{\alpha \beta} \tilde{\nabla}_{\alpha} \tilde{\nabla}_{\beta} \tilde{\nabla}^{n} g \\
& +\sum_{\sum_{s=1}^{n+2} k_{s} \leq n+2,0 \leq k_{s} \leq n+1} \tilde{\nabla}^{k_{1}} g * \cdots * \tilde{\nabla}^{k_{n+2}} g * P_{k_{1} \cdots k_{n+2}} \\
& +\sum_{\ell_{1}+\ell_{2}=n+2,1 \leq \ell_{s} \leq n+1} \tilde{\nabla}^{\ell_{1}} \phi * \tilde{\nabla}^{\ell_{2}} \phi,
\end{aligned}
$$

where $P_{k_{1} \cdots k_{n+2}}$ is a polynomial in $g, g^{-1}, \widetilde{\mathrm{Rm}}, \cdots, \tilde{\nabla}^{n} \widetilde{\mathrm{Rm}}$. Similarly, from 2.22 we can show that

$$
\begin{aligned}
\partial_{t} \tilde{\nabla}^{n} \phi= & g^{\alpha \beta} \tilde{\nabla}_{\alpha} \tilde{\nabla}_{\beta} \tilde{\nabla}^{n} \phi \\
& +\sum_{\sum_{s=1}^{n} k_{s}+\ell_{1}+\ell_{2} \leq n+2,0 \leq k_{s} \leq n, 0 \leq \ell_{1}, \ell_{2} \leq n+1} \tilde{\nabla}^{k_{1}} g * \cdots * \tilde{\nabla}^{k_{n}} g \\
& * \tilde{\nabla}^{\ell_{1}} \phi * \tilde{\nabla}^{\ell_{2}} \phi * P_{k_{1} \cdots k_{n} \ell_{1} \ell_{2} .} .
\end{aligned}
$$

Using the notion $\Theta$ defined in (3.28), we conclude from (3.57) and 3.58 that

$$
\begin{aligned}
\partial_{t} \tilde{\nabla}^{n} \boldsymbol{\Theta}= & g^{\alpha \beta} \tilde{\nabla}_{\alpha} \tilde{\nabla}_{\beta} \tilde{\nabla}^{n} \boldsymbol{\Theta} \\
& +\sum_{\sum_{s=1}^{n+2} k_{s} \leq n+2,0 \leq k_{s} \leq n+1} \tilde{\nabla}^{k_{1}} \boldsymbol{\Theta} \cdots * \tilde{\nabla}^{k_{n+2}} \boldsymbol{\Theta} * P_{k_{1} \cdots k_{s}} .
\end{aligned}
$$

The above equation is exact the equation (3.41) in [19] or the equation (69) in page 254 of [26], and, following the same argument, we obtain $\left|\tilde{\nabla}^{n} \boldsymbol{\Theta}\right|_{\tilde{g}}^{2} \lesssim 1$ on $B_{\tilde{g}}\left(x_{0}, r+\frac{\delta}{n+1}\right) \times[0, T]$, where $\lesssim$ depends only on $n, m, r, \delta, T, \tilde{g}, k_{1}$.

Fix a point $x_{0} \in M$ and choose a family of domains $\left(D_{k}\right)_{k \in \mathbf{N}}$ on $M$ such that for each $k, \partial D_{k}$ is a compact smooth $(m-1)$-dimensional submanifold of $M$ and

$$
\bar{D}_{k}=D_{k} \cup \partial D_{k} \text { is a compact subset of } M, \quad B_{\tilde{g}}\left(x_{0}, k\right) \subset D_{k} .
$$

By the same argument used in 26, together with Theorem 3.5 and Lemma 3.7, we have 
Theorem 3.8. Suppose that $(M, \tilde{g})$ is a smooth complete Riemannian manifold of dimension $m$ with $|\widetilde{\mathrm{Rm}}|_{\tilde{g}}^{2} \leq k_{0}$ and $\tilde{\phi}$ be a smooth function satisfying $|\phi|^{2}+|\tilde{\nabla} \phi|_{\tilde{g}}^{2} \leq$ $k_{1}$ on $M$. There exists a positive constant $T=T\left(m, k_{0}, k_{1}, \alpha_{1}, \beta_{1}, \beta_{2}\right)$ such that the flow

$$
\begin{aligned}
\partial_{t} g_{i j} & =-2 R_{i j}+2 \alpha_{1} \nabla_{i} \phi \nabla_{j} \phi+\nabla_{i} V_{j}+\nabla_{j} V_{i}, \\
\partial_{t} \phi & =\Delta \phi+\beta_{1}|\nabla \phi|_{g}^{2}+\beta_{2} \phi+\langle V, \nabla \phi\rangle_{g}, \\
(g(0), \phi(0)) & =(\tilde{g}, \tilde{\phi}),
\end{aligned}
$$

has a smooth solution $(g(t), \phi(t))$ on $M \times[0, T]$ that satisfies the estimate

$$
\left(1-\frac{1}{80000\left(1+\alpha_{1}^{2}+\beta_{1}^{2}\right) m^{10}}\right) \tilde{g} \leq g(t) \leq\left(1+\frac{1}{80000\left(1+\alpha_{1}^{2}+\beta_{1}^{2}\right) m^{10}}\right) \tilde{g}
$$

on $M \times[0, T]$. Moreover $|\phi(t)|^{2} \lesssim 1$ where $\lesssim$ depends on $m, k_{0}, \alpha_{1}, \beta_{1}, \beta_{2}$.

Proof. By the regularity of the flow and applying Corollary to $D_{k}$, we have $|\nabla \phi|_{g}^{2} \lesssim$ 1 on $D_{k}$, where $\lesssim$ depends only on $k_{1}, \alpha_{1}, \beta_{2}$; then $|\tilde{\nabla} \phi|_{\tilde{g}}^{2} \lesssim 1$ on $D_{k}$, where $\lesssim$ depends only on $m, k_{1}, \alpha_{1}, \beta_{1}, \beta_{2}$. Letting $k \rightarrow \infty$, we see that $|\tilde{\nabla} \phi|_{\tilde{g}}^{2} \lesssim 1$ on $M$, where $\lesssim$ depends only on $m, k_{1}, \alpha_{1}, \beta_{1}, \beta_{2}$. In particular, $|\phi(t)|^{2} \lesssim 1$ on $M$, where $\lesssim$ depends only on $m, k_{0}, \alpha_{1}, \beta_{1}, \beta_{2}$.

3.3. First order derivative estimates. Let $\tilde{\phi}$ be a smooth function on a smooth complete Riemannian manifold $(M, \tilde{g})$ of dimension $m$. Assume

$$
|\widetilde{\operatorname{Rm}}|_{\tilde{g}}^{2} \leq k_{0}, \quad|\tilde{\phi}|^{2}+|\tilde{\nabla} \tilde{\phi}|_{\tilde{g}}^{2} \leq k_{1}, \quad\left|\tilde{\nabla}{ }^{2} \tilde{\phi}\right|_{\tilde{g}}^{2} \leq k_{2}
$$

on $M$. Let $(g(t), \phi(t)), T$ be obtained in Theorem 3.8 and

$$
\delta:=\frac{1}{80000\left(1+\alpha_{1}^{2}+\beta_{1}^{2}\right) m^{10}} .
$$

Then

$$
(1-\delta) \tilde{g} \leq g(t) \leq(1+\delta) \tilde{g}
$$

on $M \times[0, T]$. As in [26], define

$$
h_{i j}:=g_{i j}-\tilde{g}, \quad H_{i j}:=\frac{1}{\delta} h_{i j} .
$$

Then

$$
\partial_{t} h_{i j}=g^{\alpha \beta} \tilde{\nabla}_{\alpha} \tilde{\nabla}_{\beta} h_{i j}+A_{i j}
$$

where

$$
\begin{aligned}
A_{i j}= & g^{\alpha \beta} g_{i p} \tilde{g}^{p q} \tilde{R}_{j \alpha q \beta}+g^{\alpha \beta} g_{j p} \tilde{g}^{p q} \tilde{R}_{i \alpha q \beta}+2 \alpha_{1} \tilde{\nabla}_{i} \phi \tilde{\nabla}_{j} \phi \\
& +\frac{1}{2} g^{\alpha \beta} g^{p q}\left(\tilde{\nabla}_{i} h_{p \alpha}+2 \tilde{\nabla}_{\alpha} h_{j p} \tilde{\nabla}_{q} h_{i \beta}-2 \tilde{\nabla}_{\alpha} h_{j p} \tilde{\nabla}_{\beta} h_{i q}\right. \\
& \left.+-2 \tilde{\nabla}_{j} h_{p \alpha} \tilde{\nabla}_{\beta} h_{i q}-2 \tilde{\nabla}_{i} h_{p \alpha} \tilde{\nabla}_{\beta} h_{j q}\right) .
\end{aligned}
$$

From $\delta<1 / 2$ and 3.60 we have from 3.64 that

$$
-\left(8 m \sqrt{k_{0}}+20|\tilde{\nabla} h|_{\tilde{g}}^{2}\right) \tilde{g} \leq A_{i j} \leq\left(8 m \sqrt{k_{0}}+20|\tilde{\nabla} h|_{\tilde{g}}^{2}\right) \tilde{g}
$$

on $M \times[0, T]$. Therefore

$$
\partial_{t} H_{i j}=g^{\alpha \beta} \tilde{\nabla}_{\alpha} \tilde{\nabla}_{\beta} H_{i j}+B_{i j}, \quad H(0)=0,
$$


where $B_{i j}:=A_{i j} / \delta$ satisfying

$$
-\left(\frac{8 m \sqrt{k_{0}}}{\delta}+20 \delta|\tilde{\nabla} H|_{\tilde{g}}^{2}\right) \tilde{g} \leq B_{i j} \leq\left(\frac{8 m \sqrt{k_{0}}}{\delta}+20 \delta|\tilde{\nabla} H|_{\tilde{g}}^{2}\right) \tilde{g}
$$

on $M \times[0, T]$. As in [19], define

$$
\psi:=\phi-\tilde{\phi}, \quad \Psi:=\delta \psi .
$$

Then

$$
\partial_{t} \psi=g^{\alpha \beta} \tilde{\nabla}_{\alpha} \tilde{\nabla}_{\beta} \psi+C,
$$

where

$$
C:=g^{\alpha \beta} \tilde{\nabla}_{\alpha} \tilde{\nabla}_{\beta} \tilde{\phi}+\beta_{1}|\tilde{\nabla} \tilde{\phi}|_{g}^{2}+\beta_{2} \tilde{\phi}+\beta_{1}|\tilde{\nabla} \psi|_{g}^{2}+\beta_{2} \psi+2 \beta_{1}\langle\tilde{\nabla} \psi, \tilde{\nabla} \tilde{\phi}\rangle_{g} .
$$

From $\delta<1 / 2,3.60$ and the proof of Theorem 3.8, we have from (3.69) that

$$
|C| \leq 2 m \sqrt{k_{0}}+2 \beta_{1} k_{1}+\beta_{2} \sqrt{k_{1}}+\beta_{2}|\psi|+2 \beta_{1}|\tilde{\nabla} \psi|_{\tilde{g}}^{2} \lesssim 1
$$

where $\lesssim$ depends on $m, k_{0}, k_{1}, \alpha_{1}, \beta_{1}, \beta_{2}$. Consequently,

$$
\begin{aligned}
\partial_{t} H_{i j} & =g^{\alpha \beta} \tilde{\nabla}_{\alpha} \tilde{\nabla}_{\beta} H_{i j}+B_{i j}, \quad H(0)=0, \\
\partial_{t} \Psi & =g^{\alpha \beta} \tilde{\nabla}_{\alpha} \tilde{\nabla}_{\beta} \Psi+D, \quad \Psi(0)=0 .
\end{aligned}
$$

Since

$$
\begin{aligned}
-\tilde{g} & \leq H(t) \leq \tilde{g} \\
\frac{1}{1+\delta} \tilde{g}^{-1} & \leq g^{-1} \leq \frac{1}{1-\delta} \tilde{g}^{-1}, \\
\left|\tilde{\nabla} g^{-1}(t)\right|_{\tilde{g}}^{2} & \leq \frac{\delta^{2}}{(1-\delta)^{4}}|\tilde{\nabla} H(t)|_{\tilde{g}}^{2} \\
|\Psi(t)|^{2} & \leq 1
\end{aligned}
$$

By the argument in [26, we arrive at

$$
\sup _{M \times[0, T]}\left(|\tilde{\nabla} H(t)|_{\tilde{g}}^{2}+|\tilde{\nabla} \Psi(t)|_{\tilde{g}}^{2}\right) \leq 1
$$

where $\lesssim$ depends only on $m, k_{0}, k_{1}, k_{1}, \alpha_{1}, \beta_{1}, \beta_{2}$.

Theorem 3.9. There exists a positive constant $T=T\left(m, k_{0}, k_{1}, \alpha_{1}, \beta_{1}, \beta_{2}\right)$ depending only on $m, k_{0}, k_{1}, \alpha_{1}, \beta_{1}, \beta_{2}$ such that

$$
\sup _{M \times[0, T]}|\tilde{\nabla} g(t)|_{\tilde{g}}^{2} \leq C, \quad \sup _{M \times[0, T]}|\tilde{\nabla} \phi|_{\tilde{g}}^{2} \leq C
$$

where $C$ is a positive constant depending only on $m, k_{0}, k_{1}, k_{2}, \alpha_{1}, \beta_{1}, \beta_{2}$.

3.4. Second order derivative estimates. In this subsection we derive the second order derivative estimates. Let $(M, \tilde{g})$ be a smooth complete Riemannian manifold of dimension $m$ and $\tilde{\phi}$ a smooth function on $M$, satisfying

$$
|\widetilde{\operatorname{Rm}}|_{\tilde{g}} \leq k_{0}, \quad|\tilde{\phi}|^{2}+|\tilde{\nabla} \tilde{\phi}|_{\tilde{g}}^{2} \leq k_{1}, \quad\left|\tilde{\nabla}^{2} \tilde{\phi}\right|_{\tilde{g}}^{2} \leq k_{2} .
$$

From Theorem 3.8 and Theorem 3.9 , there exists a positive constant $T$ depending on $m, k_{0}, k_{1}, \alpha_{1}, \beta_{1}, \beta_{2}$ such that the flow

$$
\begin{aligned}
\partial_{t} g_{i j} & =-2 R_{i j}+2 \alpha_{1} \nabla_{i} \phi \nabla_{j} \phi+\nabla_{i} V_{j}+\nabla_{j} V_{i}, \\
\partial_{t} \phi & =\Delta \phi+\beta_{1}|\nabla \phi|_{g}^{2}+\beta_{2} \phi+\langle V, \nabla \phi\rangle_{g}, \\
(g(0), \phi(0)) & =(\tilde{g}, \tilde{\phi}),
\end{aligned}
$$


has a smooth solution $(g(t), \phi(t))$ on $M \times[0, T]$ that satisfies the estimates

$$
\frac{1}{2} \tilde{g} \leq g(t) \leq 2 \tilde{g}, \quad|\tilde{\phi}|^{2} \lesssim 1, \quad|\tilde{\nabla} g|_{\tilde{g}}^{2} \lesssim 1, \quad|\tilde{\nabla} \phi|_{\tilde{g}}^{2} \lesssim 1,
$$

where $\lesssim$ (used throughout this subsection) depend only on $m, k_{0}, k_{1}, k_{2}, \alpha_{1}, \beta_{1}, \beta_{2}$.

According to Lemma 2.5 and the proof of equation (34) in page 266 of [26], we have

(3.72) $\partial_{t} \mathrm{Rm}=\Delta \mathrm{Rm}+g^{*-2} * \mathrm{Rm}^{* 2}+g^{-1} * V * \nabla \mathrm{Rm}+g^{-1} * \mathrm{Rm} * \nabla V+\nabla^{2} \phi * \nabla^{2} \phi$.

Recall the formula (28) in page 266 of [26],

$$
\partial_{t} V=\Delta V+g^{*-2} * \mathrm{Rm} * V+g^{-1} * V+* \partial_{t} g+g^{-1} * \tilde{\nabla} g * \partial_{t} g^{-1} * g .
$$

Since

$$
\partial_{t} g=g^{-1} * \mathrm{Rm}+\nabla V+\nabla \phi * \nabla \phi, \quad \partial_{t} g^{-1}=g^{-1} * g^{-1} * \partial_{t} g, \quad V=g^{-1} * \tilde{\nabla} g,
$$

it follows that

$$
\begin{array}{ll}
\partial_{t} V= & \Delta V+g^{*-3} * \tilde{\nabla} g * \mathrm{Rm}+g^{*-2} * \tilde{\nabla} g * \nabla V+g^{*-4} * g * \tilde{\nabla} g * \mathrm{Rm} \\
(3.73) \quad+g^{*-3} * g * \tilde{\nabla} g * \nabla V+g^{*-2} * \tilde{\nabla} g * \nabla \phi * \nabla \phi+g^{*-3} * g * \tilde{\nabla} g * \nabla \phi * \nabla \phi .
\end{array}
$$

Since

$$
\partial_{t} \nabla V=\nabla\left(\partial_{t} V\right)+V * \partial_{t} \Gamma, \quad \partial_{t} \Gamma=g^{-1} * \nabla\left(\partial_{t} g\right),
$$

it follows that (as the proof of the equation (99) in page 278 of [26])

$$
\begin{aligned}
\partial_{t} \nabla V= & \Delta \nabla V+g^{*-3} * \nabla \tilde{\nabla} g * \mathrm{Rm}+g^{*-3} * \tilde{\nabla} g * \nabla \mathrm{Rm}+g^{*-2} * \nabla \tilde{\nabla} g * \nabla V \\
& +g^{*-2} * \tilde{\nabla} g * \nabla^{2} V+g^{*-4} * g * \nabla \tilde{\nabla} g * \mathrm{Rm}+g^{*-4} * g * \tilde{\nabla} g * \nabla \mathrm{Rm} \\
& +g^{*-3} * g * \nabla \tilde{\nabla} g * \nabla V+g^{*-3} * g * \tilde{\nabla} g * \nabla^{2} V \\
& +g^{*-2} * \nabla \tilde{\nabla} g * \nabla \phi * \nabla \phi+g^{*-2} * \tilde{\nabla} g * \nabla \phi * \nabla^{2} \phi \\
& +g^{*-3} * g * \nabla \tilde{\nabla} g * \nabla \phi * \nabla \phi+g^{*-3} * g * \tilde{\nabla} g * \nabla \phi * \nabla^{2} \phi .
\end{aligned}
$$

By the diffeomorphisms $\left(\Psi_{t}\right)_{t \in[0, T]}$ defined by (2.17), we have

$$
\begin{aligned}
\partial_{t} \hat{g}_{i j}(x, t) & =-2 \hat{R}_{i j}(x, t)+2 \alpha_{1} \hat{\nabla}_{i} \hat{\phi}(x, t) \hat{\nabla}_{j} \hat{\phi}(x, t), \\
\partial_{t} \hat{\phi}(x, t) & =\hat{\Delta} \hat{\phi}(x, t)+\beta_{1}|\hat{\nabla} \hat{\phi}|_{\hat{g}}^{2}(x, t)+\beta_{2} \hat{\phi}(x, t),
\end{aligned}
$$

where $\hat{g}(x, t)$ and $\hat{\phi}(x, t)$ are defined by 2.16. Then

$$
\nabla_{i} \nabla_{j} \phi(x, t)=y^{\alpha}{ }_{, i} y^{\beta}{ }_{j} \hat{\nabla}_{\alpha} \hat{\nabla}_{\beta} \hat{\phi}(y, t), \quad y^{\alpha}{ }_{, i}:=\frac{\partial}{\partial x^{i}} y^{\alpha},
$$

and hence

$$
\begin{aligned}
\partial_{t} \nabla_{i} \nabla_{j} \phi(x, t)= & y^{\alpha}{ }_{, i} y^{\beta}{ }_{, j} \partial_{t} \hat{\nabla}_{\alpha} \hat{\nabla}_{\beta} \hat{\phi}(y, t)+y^{\alpha}{ }_{, i} y^{\beta}{ }_{, j} \partial_{t} y^{\gamma} \frac{\partial}{\partial y^{\gamma}} \hat{\nabla}_{\alpha} \hat{\nabla}_{\beta} \hat{\phi}(y, t) \\
& +\partial_{t}\left(y^{\alpha}{ }_{, i} y^{\beta}{ }_{, j}\right) \hat{\nabla}_{\alpha} \hat{\nabla}{ }_{\beta} \hat{\phi}(y, t) .
\end{aligned}
$$

By Lemma 2.7, we have

$$
\begin{aligned}
& y^{\alpha}{ }_{i} y^{\beta}{ }_{j} \partial_{t} \hat{\nabla}_{\alpha} \hat{\nabla}_{\beta} \hat{\phi}(y, t)=\Delta \nabla_{i} \nabla_{j} \phi+g^{*-2} * \mathrm{Rm} * \nabla^{2} \phi+\beta_{2} \nabla^{2} \phi \\
& +g^{-1} * \nabla \phi * \nabla \phi * \nabla^{2} \phi+g^{-1} * \nabla \phi * \nabla^{3} \phi \\
& +g^{-1} * \nabla^{2} \phi * \nabla^{2} \phi+g^{*-2} * \mathrm{Rm} * \nabla \phi * \nabla \phi \text {. }
\end{aligned}
$$


Using (17) and (18) in page 263 of [26, we can conclude that

$$
\begin{aligned}
I= & y^{\alpha}{ }_{, i} y^{\beta}{ }_{, j} \partial_{t} y^{\gamma} \frac{\partial}{\partial y^{\gamma}} \hat{\nabla}_{\alpha} \hat{\nabla}_{\beta} \hat{\phi}(y, t)=y_{, i}^{\alpha} y^{\beta}{ }_{, j} \partial_{t} y^{\gamma} \frac{\partial x^{p}}{\partial y^{\gamma}} \frac{\partial}{\partial x^{p}} \hat{\nabla}_{\alpha} \hat{\nabla}_{\beta} \hat{\phi}(y, t) \\
= & \partial_{t} y^{\gamma} \frac{\partial x^{p}}{\partial y^{\gamma}} \frac{\partial}{\partial x^{p}}\left(y^{\alpha}{ }_{, i} y^{\beta}{ }_{, j} \hat{\nabla}_{\alpha} \hat{\nabla}_{\beta} \hat{\phi}\right)-\partial_{t} y^{\gamma} \frac{\partial x^{p}}{\partial y^{\gamma}}\left(y^{\alpha}, i y^{\beta}{ }_{, j}\right)_{, p} \hat{\nabla}_{\alpha} \hat{\nabla}_{\beta} \hat{\phi} \\
= & g^{p q} V_{q} \frac{\partial}{\partial x^{p}} \nabla_{i} \nabla_{j} \phi-g^{p q} V_{q}\left(y^{\alpha}{ }_{, i p} \frac{\partial x^{s}}{\partial y^{\alpha}} \nabla_{s} \nabla_{j} \phi+y^{\beta}, j p \frac{\partial x^{s}}{\partial y^{\beta}} \nabla_{i} \nabla_{s} \phi\right), \\
J= & \partial_{t}\left(y^{\alpha}{ }_{, i} y^{\beta}{ }_{, j}\right) \hat{\nabla}_{\alpha} \hat{\nabla}_{\beta} \hat{\phi}(y, t)=\left(\partial_{t} y^{\alpha}\right)_{, i} \frac{\partial x^{s}}{\partial y^{\alpha}} \nabla_{s} \nabla_{j} \phi+\left(\partial_{t} y^{\beta}\right)_{, j} \frac{\partial x^{s}}{\partial y^{\beta}} \nabla_{i} \nabla_{s} \phi \\
= & g^{p q} V_{q}\left(y^{\alpha}{ }_{, p i} \frac{\partial x^{s}}{\partial y^{\beta}} \nabla_{s} \nabla_{j} \phi+y^{\beta}{ }_{, p j} \frac{\partial x^{s}}{\partial y^{\alpha}} \nabla_{i} \nabla_{s} \phi\right) \\
& +\left(g^{p q} V_{q}\right)_{, i} \nabla_{p} \nabla_{j} \phi+\left(g^{p q} V_{q}\right)_{, j} \nabla_{i} \nabla_{p} \phi .
\end{aligned}
$$

Consequently,

$$
\begin{aligned}
I+J & =g^{p q} V_{q} \nabla_{p} \nabla_{i} \nabla_{j} \phi+g^{p q}\left(\nabla_{i} V_{q} \nabla_{p} \nabla_{j} \phi+\nabla_{j} V_{q} \nabla_{i} \nabla_{q} \phi\right) \\
& =g^{-1} * V * \nabla^{3} \phi+g^{-1} * \nabla V * \nabla^{2} \phi .
\end{aligned}
$$

Combining those identities yields

$$
\begin{aligned}
\partial_{t} \nabla^{2} \phi= & \Delta \nabla^{2} \phi+g^{*-2} * \operatorname{Rm} * \nabla^{2} \phi+\beta_{2} \nabla^{2} \phi+g^{-1} * \nabla \phi * \nabla \phi * \nabla^{2} \phi \\
& +g^{-1} * \nabla \phi * \nabla^{3} \phi+g^{-1} * \nabla^{2} \phi * \nabla^{2} \phi+g^{*-2} * \operatorname{Rm} * \nabla \phi * \nabla \phi \\
& +g^{-1} * V * \nabla^{3} \phi+g^{-1} * \nabla V * \nabla^{2} \phi .
\end{aligned}
$$

The volume form

$$
d V:=d V_{g(t)}=\sqrt{\operatorname{det}\left(g_{i j}\right)} d x^{1} \wedge \cdots \wedge d x^{m}
$$

evolves by

$$
\partial_{t} d V=\frac{1}{2} g^{i j} \partial_{t} g_{i j} d V=\left(-R+\alpha_{1}|\nabla \phi|_{g}^{2}+\operatorname{div}_{g} V\right) d V .
$$

In particular, $d V=d \tilde{V}$. For any point $x_{0} \in M$ and any $r>0$ we denote by $B_{\tilde{g}}\left(x_{0}, r\right)$ the metric ball with respect to $\tilde{g}$. Recall the definition

$$
\Theta=(g, \phi) .
$$

Lemma 3.10. We have

$$
\int_{0}^{T}\left(\int_{B_{\tilde{g}}\left(x_{0}, r\right)}\left|\tilde{\nabla}^{2} \Theta\right| \frac{\tilde{g}}{\tilde{g}} d \tilde{V}\right) d t \lesssim 1
$$

where $\lesssim$ depends on $m, r, k_{0}, k_{1}, \alpha_{1}, \beta_{1}, \beta_{2}$.

Proof. As in the proof of Lemma 6.2 in [26, we chose a cutoff function $\xi(x) \in$ $C_{0}^{\infty}(M)$ such that $|\tilde{\nabla} \xi|_{\tilde{g}} \leq 8$ in $M$ and

$$
\begin{aligned}
& \xi=1 \text { in } B_{\tilde{g}}\left(x_{0}, r\right), \\
& \xi=0 \text { in } M \backslash B_{\tilde{g}}\left(x_{0}, r+\frac{1}{2}\right), \\
& 0 \leq \xi \leq 1 \text { in } M .
\end{aligned}
$$


Since $m=g^{i j} g_{i j}$, it follows that the constant 1 can be replaced by $g^{-1} * g$. From (3.16) we have

$$
\begin{aligned}
\partial_{t} \tilde{\nabla} g= & g^{\alpha \beta} \tilde{\nabla}_{\alpha} \tilde{\nabla}_{\beta} \tilde{\nabla} g+g^{-1} * g * \tilde{\nabla} \widetilde{\mathrm{Rm}}+g^{*-2} * g * \tilde{\nabla} g * \widetilde{\mathrm{Rm}} \\
& +g^{*-2} * \tilde{\nabla} g * \tilde{\nabla}^{2} g+g^{*-3} *(\tilde{\nabla} g)^{* 3}+\tilde{\nabla} \phi * \tilde{\nabla}^{2} \phi .
\end{aligned}
$$

Therefore integrating $|\tilde{\nabla} g|_{\tilde{g}}^{2} \xi^{2}$ over the ball $B_{\tilde{g}}\left(x_{0}, r+1\right)$ implies

$$
\begin{aligned}
I:= & \frac{d}{d t} \int_{B_{\tilde{g}}\left(x_{0}, r+1\right)}|\tilde{\nabla} g|_{\tilde{g}}^{2} \xi^{2} d \tilde{V}=2 \int_{B_{\tilde{g}}\left(x_{0}, r+1\right)}\left\langle\tilde{\nabla} g, \partial_{t} \tilde{\nabla} g\right\rangle_{\tilde{g}} \xi^{2} d \tilde{V} \\
= & 2 \int_{B_{\tilde{g}\left(x_{0}, r+1\right)}\left\langle\tilde{\nabla} g, g^{\alpha \beta} \tilde{\nabla}_{\alpha} \tilde{\nabla}_{\beta} \tilde{\nabla} g\right\rangle_{\tilde{g}} \xi^{2} d \tilde{V}} \\
& +\int_{B_{\tilde{g}}\left(x_{0}, r+1\right)} g^{-1} * g * \tilde{\nabla} g * \tilde{\nabla} \widetilde{\operatorname{Rm}} \xi^{2} d \tilde{V}+\int_{B_{\tilde{g}}\left(x_{0}, r+1\right)}\left[g^{*-2} * g * \tilde{\nabla} g * \widetilde{\mathrm{Rm}}\right. \\
& \left.+g^{*-2} * \tilde{\nabla} g * \tilde{\nabla}^{2} g+g^{*-3} *(\tilde{\nabla} g)^{* 3}+\tilde{\nabla} \phi * \tilde{\nabla}^{2} \phi\right] * \tilde{\nabla} g \xi^{2} d \tilde{V} \\
:= & I_{1}+I_{2}+I_{3} .
\end{aligned}
$$

Now the following computations are similar to that given in [19]. For convenience, we give a self-contained proof. Using (3.70) and (3.71) we get

$$
\begin{aligned}
& \tilde{\nabla} g *\left[g^{*-2} * g * \tilde{\nabla} g * \widetilde{\operatorname{Rm}}+g^{*-2} * \tilde{\nabla} g * \tilde{\nabla}^{2} g+g^{*-3} *(\tilde{\nabla} g)^{* 3}+\tilde{\nabla} \phi * \tilde{\nabla}^{2} \phi\right] \\
\lesssim & |\tilde{\nabla} g|_{\tilde{g}}^{2}+|\tilde{\nabla} g|_{\tilde{g}}^{2}\left|\tilde{\nabla}^{2} g\right|_{\tilde{g}}+|\tilde{\nabla} g|_{\tilde{g}}^{4}+|\tilde{\nabla} g|_{\tilde{g}}|\tilde{\nabla} \phi|_{\tilde{g}}\left|\tilde{\nabla}^{2} \phi\right|_{\tilde{g}} \\
\lesssim & 1+\left|\tilde{\nabla}^{2} g\right|_{\tilde{g}}+\left|\tilde{\nabla}^{2} \phi\right|_{\tilde{g}},
\end{aligned}
$$

and then

$$
I_{3} \lesssim \int_{B_{\tilde{g}\left(x_{0}, r+1\right)}}\left(1+\left|\tilde{\nabla}^{2} g\right|_{\tilde{g}}+\left|\tilde{\nabla}^{2} \phi\right|_{\tilde{g}}\right) \xi^{2} d \tilde{V} .
$$

By the Bishop-Gromov volume comparison (see 9]), we have

$$
\int_{B_{\tilde{g}}\left(x_{0}, r+1\right)} d \tilde{V} \lesssim 1
$$

By the estimate $(3.78)$, the $I_{3}$-term can be rewritten as

$$
I_{3} \lesssim 1+\int_{B_{\tilde{g}}\left(x_{0}, r+1\right)}\left(\left|\tilde{\nabla}^{2} g\right|_{\tilde{g}}+\left|\tilde{\nabla}^{2} \phi\right|_{\tilde{g}}\right) \xi^{2} d \tilde{V} .
$$

The $I_{1}$-term and $I_{2}$-term were computed in [26] (see (54) and (58) in page 270)

$$
\begin{aligned}
I_{1} & \leq-\frac{1}{2} \int_{B_{\tilde{g}}\left(x_{0}, r+1\right)}\left|\tilde{\nabla}^{2} g\right|_{\tilde{g}}^{2} \xi^{2} d \tilde{V}+C_{1}, \\
I_{2} & \lesssim 1+\int_{B_{\tilde{g}\left(x_{0}, r+1\right)}\left|\tilde{\nabla}^{2} g\right|_{\tilde{g}} \xi d \tilde{V} .}
\end{aligned}
$$

From 3.79, (3.80), and (3.81), we arrive at

$$
I \leq-\frac{1}{4} \int_{B_{\tilde{g}}\left(x_{0}, r+1\right)}\left|\tilde{\nabla}^{2} g\right|_{\tilde{g}}^{2} \xi^{2} d \tilde{V}+C_{2} \int_{B_{\tilde{g}}\left(x_{0}, r+1\right)}\left|\tilde{\nabla}^{2} \phi\right|_{\tilde{g}} \xi d \tilde{V}+C_{2} .
$$


From (3.24) we have

$$
\begin{aligned}
\partial_{t} \tilde{\nabla} \phi= & g^{\alpha \beta} \tilde{\nabla}_{\alpha} \tilde{\nabla}_{\beta} \tilde{\nabla} \phi+g^{*-2} * \widetilde{\mathrm{Rm}} * \tilde{\nabla} \phi+g^{*-2} * \tilde{\nabla} g * \tilde{\nabla}^{2} \phi \\
& +g^{*-2} * \tilde{\nabla} g *(\tilde{\nabla} \phi)^{* 2}+g^{-1} * \tilde{\nabla} \phi * \tilde{\nabla}^{2} \phi+\beta_{2} \tilde{\nabla} \phi .
\end{aligned}
$$

Therefore integrating $|\tilde{\nabla} \phi|_{\tilde{g}}^{2} \xi^{2}$ over the ball $B_{\tilde{g}}\left(x_{0}, r+1\right)$ implies

$$
\begin{aligned}
J:= & \frac{d}{d t} \int_{B_{\tilde{g}}\left(x_{0}, r+1\right)}|\tilde{\nabla} \phi|_{\tilde{g}}^{2} \xi^{2} d \tilde{V}=2 \int_{B_{\tilde{g}}\left(x_{0}, r+1\right)}\left\langle\tilde{\nabla} \phi, \partial_{t} \tilde{\nabla} \phi\right\rangle_{\tilde{g}} \xi^{2} d \tilde{V} \\
= & 2 \int_{B_{\tilde{g}}\left(x_{0}, r+1\right)}\left\langle\tilde{\nabla} \phi, g^{\alpha \beta} \tilde{\nabla}_{\alpha} \tilde{\nabla}_{\beta} \tilde{\nabla} \phi\right\rangle_{\tilde{g}} \xi^{2} d \tilde{V}+\int_{B_{\tilde{g}\left(x_{0}, r+1\right)}} \xi^{2}\left[g^{*-2} * \widetilde{\operatorname{Rm}} * \tilde{\nabla} \phi\right. \\
& \left.+g^{*-2} * \tilde{\nabla} g * \tilde{\nabla}^{2} \phi+g^{*-2} * \tilde{\nabla} g *(\tilde{\nabla} \phi)^{* 2}+g^{-1} * \tilde{\nabla} \phi * \tilde{\nabla}^{2} \phi+\tilde{\nabla} \phi\right] \tilde{\nabla} \phi d \tilde{V} \\
:= & J_{1}+J_{2} .
\end{aligned}
$$

Using (3.70) and (3.71), we get

$$
\begin{aligned}
& {\left[g^{*-2} * \widetilde{\operatorname{Rm}} * \tilde{\nabla} \phi+g^{*-2} * \tilde{\nabla} g * \tilde{\nabla}^{2} \phi+g^{*-2} * \tilde{\nabla} g *(\tilde{\nabla} \phi)^{* 2}\right.} \\
& \left.+g^{-1} * \tilde{\nabla} \phi * \tilde{\nabla}^{2} \phi+\tilde{\nabla} \phi\right] * \tilde{\nabla} \phi \\
\lesssim & |\tilde{\nabla} \phi|_{\tilde{g}}^{2}+|\tilde{\nabla} g|_{\tilde{g}}|\tilde{\nabla} \phi|_{\tilde{g}}\left|\tilde{\nabla}^{2} \phi\right|_{\tilde{g}}+|\tilde{\nabla} g|_{\tilde{g}}|\tilde{\nabla} \phi|_{\tilde{g}}^{3}+|\tilde{\nabla} \phi|_{\tilde{g}}^{2}\left|\tilde{\nabla}^{2} \phi\right|_{\tilde{g}}+|\tilde{\nabla} \phi|_{\tilde{g}}^{2} \\
\lesssim & 1+\left|\tilde{\nabla}^{2} \phi\right|_{\tilde{g}}
\end{aligned}
$$

By the estimate (3.78), the $J_{2}$-term can be bounded by

$$
J_{2} \lesssim 1+\int_{B_{\tilde{g}}\left(x_{0}, r+1\right)}\left|\tilde{\nabla}^{2} \phi\right|_{\tilde{g}} \xi d \tilde{V} .
$$

From the integration by parts, we obtain

$$
\begin{aligned}
J_{1}= & -2 \int_{B_{\tilde{g}\left(x_{0}, r+1\right)}} g^{\alpha \beta}\left\langle\tilde{\nabla}_{\beta} \tilde{\nabla} \phi, \tilde{\nabla}_{\alpha} \tilde{\nabla} \phi\right\rangle_{\tilde{g}} \xi^{2} d \tilde{V} \\
& +\int_{B_{\tilde{g}\left(x_{0}, r+1\right)}} \tilde{\nabla}^{2} \phi * g^{*-2} * \tilde{\nabla} g * \tilde{\nabla} \phi \xi^{2} d \tilde{V} \\
& +\int_{B_{\tilde{g}}\left(x_{0}, r+1\right)} g^{-1} * \tilde{\nabla}^{2} \phi * \tilde{\nabla} \phi * \xi * \tilde{\nabla} \xi d \tilde{V}
\end{aligned}
$$

Since

by 3.71 , and

$$
g^{\alpha \beta}\left\langle\tilde{\nabla}_{\beta} \tilde{\nabla} \phi, \tilde{\nabla}_{\alpha} \tilde{\nabla} \phi\right\rangle_{\tilde{g}} \geq \frac{1}{2}\left|\tilde{\nabla}^{2} \phi\right|_{\tilde{g}}^{2}
$$

$$
\begin{aligned}
\int_{B_{\tilde{g}}\left(x_{0}, r+1\right)} \tilde{\nabla}^{2} \phi * g^{*-2} * \tilde{\nabla} g * \tilde{\nabla} \phi \xi^{2} d \tilde{V} & \lesssim \int_{B_{\tilde{g}\left(x_{0}, r+1\right)}\left|\tilde{\nabla}^{2} \phi\right|_{\tilde{g}} \xi d \tilde{V}} \\
\int_{B_{\tilde{g}}\left(x_{0}, r+1\right)} g^{-1} * \tilde{\nabla}^{2} \phi * \tilde{\nabla} \phi * \xi * \tilde{\nabla} \xi d \tilde{V} & \lesssim \int_{B_{\tilde{g}\left(x_{0}, r+1\right)}\left|\tilde{\nabla}^{2} \phi\right|_{\tilde{g}} \xi d \tilde{V}}
\end{aligned}
$$

it follows that

$$
J_{1} \leq-\int_{B_{\tilde{g}}\left(x_{0}, r+1\right)}\left|\tilde{\nabla}^{2} \phi\right|_{\tilde{g}}^{2} \xi^{2} d \tilde{V}+C_{3} \int_{B_{\tilde{g}}\left(x_{0}, r+1\right)}\left|\tilde{\nabla}^{2} \phi\right|_{\tilde{g}} \xi d \tilde{V} .
$$


From 3.83 and 3.84 ,

$$
J \leq-\frac{1}{2} \int_{B_{\tilde{g}}\left(x_{0}, r+1\right)}\left|\tilde{\nabla}^{2} \phi\right|_{\tilde{g}}^{2} \xi^{2} d \tilde{V}+C_{4} .
$$

Together with 3.82 , we arrive at

$$
I+J \leq-\frac{1}{4} \int_{B_{\tilde{g}\left(x_{0}, r+1\right)}}\left(\left|\tilde{\nabla}^{2} g\right|_{\tilde{g}}^{2}+\left|\tilde{\nabla}^{2} \phi\right|_{\tilde{g}}^{2}\right) \xi^{2} d \tilde{V}+C_{5} .
$$

Thus

$$
\frac{d}{d t} \int_{B_{\tilde{g}}\left(x_{0}, r+1\right)}|\tilde{\nabla} \Theta|_{\tilde{g}}^{2} \xi^{2} d \tilde{V} \leq-\frac{1}{4} \int_{B_{\tilde{g}}\left(x_{0}, r+1\right)}\left|\tilde{\nabla}^{2} \Theta\right|_{\tilde{g}}^{2} \xi^{2} d \tilde{V}+C_{5} .
$$

Integrating $(3.86)$ over $[0, T]$ implies

$$
\int_{0}^{T}\left(\int_{B_{\tilde{g}}\left(x_{0}, r+1\right)}\left|\tilde{\nabla}^{2} \Theta\right|_{\tilde{g}}^{2} \xi^{2} d \tilde{V}\right) d t \lesssim 1
$$

Since $\xi=1$ on $B_{\tilde{g}}\left(x_{0}, r\right)$, the above estimate yields the desired inequality.

Using (3.71) we have

$$
\left|\tilde{\nabla}^{2} g\right|_{g}^{2} \leq 16\left|\tilde{\nabla}^{2} g\right|_{\tilde{g}}^{2}, \quad\left|\tilde{\nabla}^{2} \phi\right|_{g}^{2} \leq 4\left|\tilde{\nabla}^{2} \phi\right|_{\tilde{g}}^{2}, \quad d V \leq 2^{m / 2} d \tilde{V},
$$

on $M \times[0, T]$ and then

$$
\int_{0}^{T}\left(\int_{B_{\tilde{g}}\left(x_{0}, r\right)}\left|\tilde{\nabla}^{2} \Theta\right|_{g}^{2} d V\right) d t \lesssim \int_{0}^{T}\left(\int_{B_{\tilde{g}}\left(x_{0}, r\right)}\left|\tilde{\nabla}^{2} \boldsymbol{\Theta}\right|_{\tilde{g}}^{2} d \tilde{V}\right) d t .
$$

By (66) in page 272 of [26], we have

$$
|\nabla \tilde{\nabla} g|_{g}^{2} \leq 2\left|\tilde{\nabla}^{2} g\right|_{g}^{2}+C_{6}
$$

on the other hand, by $\nabla \tilde{\nabla} \phi=\tilde{\nabla}^{2} \phi+g^{-1} * \tilde{\nabla} g * \tilde{\nabla} \phi$, we get

$$
|\nabla \tilde{\nabla} \phi|_{g}^{2} \leq 2\left|\tilde{\nabla}^{2} \phi\right|_{g}^{2}+C_{7}
$$

Thus

$$
\int_{0}^{T}\left(\int_{B_{\tilde{g}}\left(x_{0}, r\right)}|\nabla \tilde{\nabla} \Theta|_{g}^{2} d V\right) d t \lesssim 1+\int_{0}^{T}\left(\int_{B_{\tilde{g}}\left(x_{0}, r\right)}\left|\tilde{\nabla}^{2} \Theta\right|_{g}^{2} d V\right) d t .
$$

Therefore,

Lemma 3.11. We have

$$
\int_{0}^{T}\left(\int_{B_{\tilde{g}}\left(x_{0}, r\right)}\left(\left|\tilde{\nabla}^{2} \boldsymbol{\Theta}\right|_{g}^{2}+|\nabla \tilde{\nabla} \boldsymbol{\Theta}|_{g}^{2}\right) d V\right) d t \lesssim 1 .
$$

where $\lesssim$ depends on $m, r, k_{0}, k_{1}, \alpha_{1}, \beta_{1}, \beta_{2}$.

We now prove the integral estimates for $\mathrm{Rm}, \nabla^{2} \phi$, and $\nabla V$. The similar results were proved by Shi [26] for the Ricci flow and List [19] for the Ricci flow coupled with the heat flow.

Lemma 3.12. We have

$$
\int_{B_{\tilde{g}}\left(x_{0}, r\right)}\left(|\mathrm{Rm}|_{g}^{2}+\left|\nabla^{2} \phi\right|_{g}^{2}+|\nabla V|_{g}^{2}\right) d V \lesssim 1
$$

where $\lesssim$ depends on $m, r, k_{0}, k_{1}, k_{2}, \alpha_{1}, \beta_{1}, \beta_{2}$. 
Proof. Keep to use the same cutoff function $\xi(x)$ introduced in the proof of Lemma 3.10 From $|\mathrm{Rm}|_{g}^{2}=g^{i \alpha} g^{j \beta} g^{k \gamma} g^{\ell \delta} R_{i j k \ell} R_{\alpha \beta \gamma \delta}$, we get

$$
\partial_{t}|\mathrm{Rm}|_{g}^{2}=2\left\langle\mathrm{Rm}, \partial_{t} \mathrm{Rm}\right\rangle_{g}+\mathrm{Rm}^{* 2} * g^{*-3} * \partial_{t} g^{-1}
$$

and

$$
\begin{aligned}
& \int_{B_{\tilde{g}}\left(x_{0}, r+1\right)}|\mathrm{Rm}|_{g}^{2} \xi^{2} d V \\
= & \int_{B_{\tilde{g}}\left(x_{0}, r+1\right)}|\widetilde{\mathrm{Rm}}|_{\tilde{g}}^{2} \xi^{2} d \tilde{V}+\int_{0}^{t}\left(\frac{d}{d t} \int_{B_{\tilde{g}}\left(x_{0}, r+1\right)}|\mathrm{Rm}|_{g}^{2} \xi^{2} d V\right) d t \\
(3.87)= & \int_{B_{\tilde{g}}\left(x_{0}, r+1\right)}|\widetilde{\mathrm{Rm}}|_{\tilde{g}}^{2} \xi^{2} d \tilde{V}+\int_{0}^{t}\left(\int_{B_{\tilde{g}}\left(x_{0}, r+1\right)}|\mathrm{Rm}|_{g}^{2} \xi^{2} \partial_{t} d V\right) d t \\
& +\int_{0}^{t}\left[\int_{B_{\tilde{g}}\left(x_{0}, r+1\right)}\left(2\left\langle\mathrm{Rm}, \partial_{t} \mathrm{Rm}\right\rangle_{g}+g^{*-3} * \mathrm{Rm}^{* 2} * \partial_{t} g^{-1}\right) \xi^{2} d V\right] d t
\end{aligned}
$$

By the estimate 3.78 we have

$$
\int_{B_{\tilde{g}}\left(x_{0}, r+1\right)}|\widetilde{\mathrm{Rm}}|_{\tilde{g}}^{2} \xi^{2} d \tilde{V} \lesssim 1 .
$$

Using (3.76) implies

$$
\begin{aligned}
\int_{0}^{t} \int_{B_{\tilde{g}}\left(x_{0}, r+1\right)}|\mathrm{Rm}|_{g}^{2} \xi^{2} \partial_{t} d V d t= & \int_{0}^{t} \int_{B_{\tilde{g}}\left(x_{0}, r+1\right)} g^{*-4} * \mathrm{Rm}^{* 2} *\left(g^{*-2} * \mathrm{Rm}\right. \\
& \left.+g^{-1} *(\nabla \phi)^{* 2}+g^{-1} * \nabla V\right) \xi^{2} d V d t \\
= & \int_{0}^{t} \int_{B_{\tilde{g}}\left(x_{0}, r+1\right)}\left(g^{*-5} * \mathrm{Rm}^{* 2} *(\nabla \phi)^{* 2}\right. \\
& \left.+g^{*-6} * \mathrm{Rm}^{* 3}+g^{*-5} * \mathrm{Rm}^{* 2} * \nabla V\right) \xi^{2} d V d t .
\end{aligned}
$$

By the evolution

$$
\partial_{t} g^{-1}=g^{*-3} * \mathrm{Rm}+g^{*-2} * \nabla V+g^{*-2} *(\nabla \phi)^{* 2},
$$

above (3.73), we get

$$
\begin{aligned}
& \int_{0}^{t} \int_{B_{\tilde{g}}\left(x_{0}, r+1\right)} g^{*-3} * \mathrm{Rm}^{* 2} * \partial_{t} g^{-1} \xi^{2} d V d t \\
= & \int_{0}^{t} \int_{B_{\tilde{g}}\left(x_{0}, r+1\right)} g^{*-5} * \mathrm{Rm}^{* 2} *\left(g^{-1} * \mathrm{Rm}+\nabla V+(\nabla \phi)^{* 2}\right) \xi^{2} d V d t .
\end{aligned}
$$

According to 3.72 ,

$$
\begin{aligned}
\text { (3.91) } & 2 \int_{0}^{t} \int_{B_{\tilde{g}}\left(x_{0}, r+1\right)}\left\langle\mathrm{Rm}, \partial_{t} \mathrm{Rm}\right\rangle_{g} \xi^{2} d V_{t} d t \\
= & 2 \int_{0}^{t} \int_{B_{\tilde{g}}\left(x_{0}, r+1\right)}\langle\mathrm{Rm}, \Delta \mathrm{Rm}\rangle_{g} \xi^{2} d V d t+\int_{0}^{t} \int_{B_{\tilde{g}}\left(x_{0}, r+1\right)}\left(g^{*-4} * \mathrm{Rm} *\left(\nabla^{2} \phi\right)^{* 2}\right. \\
& \left.+g^{*-5} * \mathrm{Rm}^{* 2} * \nabla V+g^{*-5} * V * \mathrm{Rm} * \nabla \mathrm{Rm}+g^{*-6} * \mathrm{Rm}^{* 3}\right) \xi^{2} d V d t .
\end{aligned}
$$


Substituting (3.88), 3.89, 3.90, and 3.91), into (3.87), we arrive at

$$
\begin{aligned}
& \int_{B_{\tilde{g}}\left(x_{0}, r+1\right)}|\mathrm{Rm}|_{g}^{2} \xi^{2} d V \\
\leq & C_{1}+2 \int_{0}^{t} \int_{B_{\tilde{g}}\left(x_{0}, r+1\right)}\langle\mathrm{Rm}, \Delta \mathrm{Rm}\rangle_{g} \xi^{2} d V d t+\int_{0}^{t} \int_{B_{\tilde{g}}\left(x_{0}, r+1\right)}\left(g^{*-6} * \mathrm{Rm}^{* 3}\right. \\
(3.92)+g^{*-5} * \mathrm{Rm}^{* 2} * \nabla V+g^{*-5} * V * \mathrm{Rm} * \nabla \mathrm{Rm} & \left.+g^{*-5} * \mathrm{Rm}^{* 2} *(\nabla \phi)^{* 2}+g^{*-4} * \mathrm{Rm} *\left(\nabla^{2} \phi\right)^{* 2}\right) \xi^{2} d V d t
\end{aligned}
$$

where $C_{1}$ is a uniform positive constant independent of $t$ and $x_{0}$. The second term on the right-hand side of 3.92 was estimated in 26] (equation (78) in page 274):

$$
\begin{gathered}
2 \int_{0}^{t} \int_{B_{\tilde{g}}\left(x_{0}, r+1\right)}\langle\mathrm{Rm}, \Delta \mathrm{Rm}\rangle_{g} \xi^{2} d V d t \\
(3.93) \leq-\frac{3}{2} \int_{0}^{t} \int_{B_{\tilde{g}}\left(x_{0}, r+1\right)}|\nabla \mathrm{Rm}|_{g}^{2} \xi^{2} d V_{t} d t+C_{2} \int_{0}^{t} \int_{B_{\tilde{g}}\left(x_{0}, r+1\right)}|\mathrm{Rm}|_{g}^{2} d V d t .
\end{gathered}
$$

Using $V=g^{-1} * \tilde{\nabla} g$ and 3.71 , as showed in [26] (equations (80), (81), (88), pages 275-277), we obtain

$$
\begin{aligned}
& \int_{0}^{t} \int_{B_{\tilde{g}}\left(x_{0}, r+1\right)} g^{*-5} * V * \mathrm{Rm} * \nabla \mathrm{Rm} \xi^{2} d V d t \\
(3.94) \leq & \frac{1}{4} \int_{0}^{t} \int_{B_{\tilde{g}}\left(x_{0}, r+1\right)}|\nabla \mathrm{Rm}|_{g}^{2} \xi^{2} d V_{t} d t+C_{3} \int_{0}^{k} \int_{B_{\tilde{g}}\left(x_{0}, r+1\right)}|\mathrm{Rm}|_{g}^{2} d V d t \\
& \left.\int_{0}^{t} \int_{B_{\tilde{g}\left(x_{0}, r+1\right)} g^{*-5} * \mathrm{Rm}^{* 2} * \nabla V \xi^{2} d V d t}\left|\frac{1}{8} \int_{0}^{t} \int_{B_{\tilde{g}}\left(x_{0}, r+1\right)}\right| \nabla \mathrm{Rm}\right|_{g} ^{2} \xi^{2} d V d t+C_{4} \int_{0}^{t} \int_{B_{\tilde{g}}\left(x_{0}, r+1\right)}|\mathrm{Rm}|_{g}^{2} d V d t \\
(3.95) \leq & \int_{0}^{t} \int_{B_{\tilde{g}}\left(x_{0}, r+1\right)} g^{*-6} * \mathrm{Rm}^{* 3} \xi^{2} d V d t \\
(3.96) \leq & \frac{1}{8} \int_{0}^{t} \int_{B_{\tilde{g}}\left(x_{0}, r+1\right)}|\nabla \mathrm{Rm}|_{g}^{2} \xi^{2} d V d t+C_{5} \int_{0}^{t} \int_{B_{\tilde{g}}\left(x_{0}, r+1\right)}|\mathrm{Rm}|_{g}^{2} d V d t .
\end{aligned}
$$

Plugging 3.93, 3.94), 3.95, 3.96 into 3.92, yields

$$
\begin{gathered}
\int_{B_{\tilde{g}}\left(x_{0}, r+1\right)}|\mathrm{Rm}|_{g}^{2} \xi^{2} d V \\
\leq-\int_{0}^{t} \int_{B_{\tilde{g}}\left(x_{0}, r+1\right)}|\nabla \mathrm{Rm}|_{g}^{2} \xi^{2} d V d t+C_{6} \int_{0}^{t} \int_{B_{\tilde{g}}\left(x_{0}, r+1\right)}|\mathrm{Rm}|_{g}^{2} d V d t+C_{7} \\
(3.97)+\int_{0}^{t} \int_{B_{\tilde{g}}\left(x_{0}, r+1\right)}\left(g^{*-5} * \mathrm{Rm}^{* 2} *(\nabla \phi)^{* 2}+g^{*-4} * \mathrm{Rm} *\left(\nabla^{2} \phi\right)^{* 2}\right) \xi^{2} d V d t .
\end{gathered}
$$

Using (3.71) and the Cauchy-Schwarz inequality, we can conclude that

$$
g^{*-5} * \mathrm{Rm}^{* 2} *(\nabla \phi)^{* 2} \lesssim|\mathrm{Rm}|_{g}^{2}|\nabla \phi|_{g}^{2}
$$


but $|\nabla \phi|_{g}^{2}=g^{i j} \tilde{\nabla}_{i} \phi \tilde{\nabla}_{j} \phi \leq 2|\tilde{\nabla} \phi|_{\tilde{g}}^{2} \lesssim 1$, the above quantity $g^{*-5} * \mathrm{Rm}^{* 2} *(\nabla \phi)^{* 2}$ is bounded from above by $|\mathrm{Rm}|_{g}^{2}$. From the equation (90) in page 277 of [26],

$$
g^{*-5} * \mathrm{Rm}^{* 2} *(\nabla \phi)^{* 2} \lesssim 1+|\nabla \tilde{\nabla} g|_{\tilde{g}}^{2} \lesssim 1+|\nabla \tilde{\nabla} g|_{g}^{2} \lesssim 1+|\nabla \tilde{\nabla} \boldsymbol{\Theta}|_{g}^{2} ;
$$

this estimate together with Lemma 3.11 gives us

$$
\int_{0}^{t} \int_{B_{\tilde{g}}\left(x_{0}, r+1\right)} g^{*-5} * \mathrm{Rm}^{* 2} *(\nabla \phi)^{* 2} \xi^{2} d V d t \lesssim 1 .
$$

To deal with the last term on the right-hand side of (3.97), we perform the integration by parts to obtain

$$
\begin{aligned}
& \int_{B_{\tilde{g}}\left(x_{0}, r+1\right)} g^{*-4} * \mathrm{Rm} *\left(\nabla^{2} \phi\right)^{* 2} \xi^{2} d V \\
& =\int_{B_{\tilde{g}}\left(x_{0}, r+1\right)} g^{*-4} * \nabla \phi *\left(\nabla \mathrm{Rm} * \nabla^{2} \phi+\mathrm{Rm} * \nabla^{3} \phi+\mathrm{Rm} * \nabla^{2} \phi * \frac{\nabla \xi}{\xi}\right) \xi^{2} d V \\
& \leq \frac{1}{2} \int_{B_{\tilde{g}}\left(x_{0}, r+1\right)}|\nabla \mathrm{Rm}|_{g}^{2} \xi^{2} d V+C_{8} \int_{B_{\tilde{g}}\left(x_{0}, r+1\right)}\left|\nabla^{2} \phi\right|_{g}^{2} d V \\
& +\epsilon \int_{B_{\tilde{g}}\left(x_{0}, r+1\right)}\left|\nabla^{3} \phi\right|_{g}^{2} \xi^{2} d V+C_{\epsilon} \int_{B_{\tilde{g}}\left(x_{0}, r+1\right)}|\mathrm{Rm}|_{g}^{2} d V \\
& +C_{9} \int_{B_{\tilde{g}}\left(x_{0}, r+1\right)}|\mathrm{Rm}|_{g}^{2} d V+C_{10} \int_{B_{\tilde{g}}\left(x_{0}, r+1\right)}\left|\nabla^{2} \phi\right|_{g}^{2} d V \\
& \leq \frac{1}{2} \int_{B_{\tilde{g}}\left(x_{0}, r+1\right)}|\nabla \mathrm{Rm}|_{g}^{2} \xi^{2} d V+\epsilon \int_{B_{\tilde{g}}\left(x_{0}, r+1\right)}\left|\nabla^{3} \phi\right|_{g}^{2} \xi^{2} d V \\
& +C_{11} \int_{B_{\tilde{g}}\left(x_{0}, r+1\right)}|\nabla \tilde{\nabla} \Theta|_{g}^{2} d V
\end{aligned}
$$

using 3.71), $\nabla^{2} \phi=\nabla \tilde{\nabla} \phi$, and $|\mathrm{Rm}|_{g}^{2} \lesssim 1+|\nabla \tilde{\nabla} g|_{g}^{2}$. Note also that the second term on the right-hand side of (3.97) is uniformly bounded by the same estimate for $|\mathrm{Rm}|_{g}^{2}$ and Lemma 3.11. Consequently,

$$
\begin{aligned}
\int_{B_{\tilde{g}}\left(x_{0}, r+1\right)}|\mathrm{Rm}|_{g}^{2} \xi^{2} d V \leq & -\frac{1}{2} \int_{0}^{t} \int_{B_{\tilde{g}}\left(x_{0}, r+1\right)}|\nabla \mathrm{Rm}|_{g}^{2} \xi^{2} d V d t \\
& +\epsilon \int_{0}^{t} \int_{B_{\tilde{g}}\left(x_{0}, r+1\right)}\left|\nabla^{3} \phi\right|_{g}^{2} \xi^{2} d V d t+C_{12} .
\end{aligned}
$$

We next establish the similar inequality for $\left|\nabla^{2} \phi\right|_{g}^{2}=g^{i k} g^{j \ell} \nabla_{i} \nabla_{j} \phi \nabla_{k} \nabla_{\ell} \phi$. Calculate $\partial_{t}\left|\nabla^{2} \phi\right|_{g}^{2}=2\left\langle\nabla^{2} \phi, \partial_{t} \nabla^{2} \phi\right\rangle_{g}+\left(\nabla^{2} \phi\right)^{* 2} * g^{-1} * \partial_{t} g^{-1}$ and

$$
\begin{aligned}
& \int_{B_{\tilde{g}\left(x_{0}, r+1\right)}}\left|\nabla^{2} \phi\right|_{g}^{2} \xi^{2} d V \\
= & \int_{B_{\tilde{g}\left(x_{0}, r+1\right)}}\left|\tilde{\nabla}^{2} \tilde{\phi}\right|_{\tilde{g}}^{2} \xi^{2} d \tilde{V}+\int_{0}^{t}\left(\frac{d}{d t} \int_{B_{\tilde{g}}\left(x_{0}, r+1\right)}\left|\nabla^{2} \phi\right|_{g}^{2} \xi^{2} d V\right) d t \\
= & \int_{B_{\tilde{g}}\left(x_{0}, r+1\right)}\left|\tilde{\nabla}^{2} \tilde{\phi}\right|_{\tilde{g}}^{2} \xi^{2} d \tilde{V}+\int_{0}^{t} \int_{B_{\tilde{g}}\left(x_{0}, r+1\right)}\left|\nabla^{2} \phi\right|_{g}^{2} \xi^{2} \partial_{t} d V d t \\
& +\int_{0}^{t} \int_{B_{\tilde{g}}\left(x_{0}, r+1\right)}\left(2\left\langle\nabla^{2} \phi, \partial_{t} \nabla^{2} \phi\right\rangle_{g}+\left(\nabla^{2} \phi\right)^{* 2} * g^{-1} * \partial_{t} g^{-1}\right) \xi^{2} d V d t
\end{aligned}
$$


Since $\left|\tilde{\nabla}^{2} \tilde{\phi}\right|_{\tilde{g}}^{2} \leq k_{2}$ by the assumption 3.70 , we have

$$
\int_{B_{\tilde{g}}\left(x_{0}, r+1\right)}\left|\tilde{\nabla}^{2} \tilde{\phi}\right|_{\tilde{g}}^{2} \xi^{2} d \tilde{V} \lesssim 1 .
$$

Using 3.76 implies

$$
\begin{aligned}
\int_{0}^{t} \int_{B_{\tilde{g}}\left(x_{0}, r+1\right)}\left|\nabla^{2} \phi\right|_{g}^{2} \xi^{2} \partial_{t} d V d t= & \int_{0}^{t} \int_{B_{\tilde{g}}\left(x_{0}, r+1\right)} g^{*-2} *\left(\nabla^{2} \phi\right)^{* 2} *\left(g^{*-2} * \mathrm{Rm}\right. \\
& \left.+g^{-1} *(\nabla \phi)^{* 2}+g^{-1} * \nabla V\right) \xi^{2} d V d t \\
= & \int_{0}^{t} \int_{B_{\tilde{g}}\left(x_{0}, r+1\right)}\left(g^{*-4} * \operatorname{Rm} *\left(\nabla^{2} \phi\right)^{* 2}\right. \\
& +g^{*-3} *(\nabla \phi)^{* 2} *\left(\nabla^{2} \phi\right)^{* 2} \\
& \left.+g^{*-3} * \nabla V *\left(\nabla^{2} \phi\right)^{* 2}\right) \xi^{2} d V d t .
\end{aligned}
$$

By the evolution equation of $\partial_{t} g^{-1}$ above 3.90 , we get

$$
\begin{aligned}
& \int_{0}^{t} \int_{B_{\tilde{g}}\left(x_{0}, r+1\right)} g^{-1} *\left(\nabla^{2} \phi\right)^{* 2} * \partial_{t} g^{-1} \xi^{2} d V d t \\
= & \int_{0}^{t} \int_{B_{\tilde{g}}\left(x_{0}, r+1\right)} g^{*-3} *\left(\nabla^{2} \phi\right)^{* 2} *\left(g^{-1} * \mathrm{Rm}+\nabla V+(\nabla \phi)^{* 2}\right) \xi^{2} d V d t .
\end{aligned}
$$

By 3.75 the third term on the right-hand side of 3.100 can be written as

$$
\begin{aligned}
& \int_{0}^{t} \int_{B_{\tilde{g}}\left(x_{0}, r+1\right)} 2\left\langle\nabla^{2} \phi, \partial_{t} \nabla^{2} \phi\right\rangle_{g} \xi^{2} d V d t \\
= & 2 \int_{0}^{t} \int_{B_{\tilde{g}}\left(x_{0}, r+1\right)}\left\langle\nabla^{2} \phi, \Delta \nabla^{2} \phi\right\rangle_{g} \xi^{2} d V d t+\int_{0}^{t} \int_{B_{\tilde{g}}\left(x_{0}, r+1\right)}\left(g^{*-2} *\left(\nabla^{2} \phi\right)^{* 2}\right. \\
(3.104)+ & +g^{*-4} * \mathrm{Rm} *\left(\nabla^{* 2} \phi\right)^{2}+g^{*-3} *(\nabla \phi)^{* 2} *\left(\nabla^{2} \phi\right)^{* 2}+g^{*-3} *\left(\nabla^{2} \phi\right)^{* 3} \\
& +g^{*-3} * \nabla \phi * \nabla^{* 2} \phi * \nabla^{3} \phi+g^{*-4} * \mathrm{Rm} *(\nabla \phi)^{* 2} * \nabla^{2} \phi \\
& \left.+g^{*-3} * V * \nabla^{2} \phi * \nabla^{3} \phi+g^{*-3} * \nabla V *\left(\nabla^{2} \phi\right)^{* 2}\right) \xi^{2} d V d t .
\end{aligned}
$$

Substituting (3.101), 3.102, 3.103), and 3.104 into 3.100, we arrive at

$$
\begin{gathered}
\int_{B_{\tilde{g}}\left(x_{0}, r+1\right)}\left|\nabla^{2} \phi\right|_{g}^{2} \xi^{2} d V \\
\leq C_{13}+2 \int_{0}^{t} \int_{B_{\tilde{g}}\left(x_{0}, r+1\right)}\left\langle\nabla^{2} \phi, \Delta \nabla^{2} \phi\right\rangle_{g} \xi^{2} d V d t \\
(3.105)+\int_{0}^{t} \int_{B_{\tilde{g}}\left(x_{0}, r+1\right)}\left(g^{*-4} * \mathrm{Rm} *\left(\nabla^{2} \phi\right)^{* 2}+g^{*-3} *(\nabla \phi)^{* 2} *\left(\nabla^{2} \phi\right)^{* 2}\right. \\
+g^{*-3} *\left(\nabla^{2} \phi\right)^{* 3}+g^{*-3} * \nabla \phi * \nabla^{2} \phi * \nabla^{3} \phi+g^{*-4} * \mathrm{Rm} *(\nabla \phi)^{* 2} * \nabla^{2} \phi \\
\left.+g^{*-3} * V * \nabla^{2} \phi * \nabla^{3} \phi+g^{*-3} * \nabla V *\left(\nabla^{2} \phi\right)^{* 2}+g^{*-2} *\left(\nabla^{2} \phi\right)^{* 2}\right) \xi^{2} d V d t .
\end{gathered}
$$


By integration by parts, we find that the first term on the right-hand side of (3.105) equals

$$
\begin{aligned}
& 2 \int_{0}^{t} B_{\tilde{g}\left(x_{0}, r+1\right)}\left\langle\xi^{2} \nabla^{2} \phi, g^{\alpha \beta} \nabla_{\alpha} \nabla_{\beta} \nabla^{2} \phi\right\rangle_{g} d V d t \\
= & -2 \int_{0}^{t} \int_{B_{\tilde{g}}\left(x_{0}, r+1\right)} g^{\alpha \beta}\left\langle\nabla_{\alpha}\left(\xi^{2} \nabla^{2} \phi\right), \nabla_{\beta} \nabla^{2} \phi\right\rangle_{g} d V d t \\
= & -2 \int_{0}^{t} \int_{B_{\tilde{g}}\left(x_{0}, r+1\right)} g^{\alpha \beta}\left\langle\nabla_{\alpha} \nabla^{2} \phi, \nabla_{\beta} \nabla^{2} \phi\right\rangle_{g} \xi^{2} d V d t \\
& +\int_{0}^{t} \int_{B_{\tilde{g}}\left(x_{0}, r+1\right)} g^{*-4} * \nabla^{2} \phi * \nabla^{3} \phi * \nabla \xi \xi d V d t \\
\leq & -\frac{3}{2} \int_{0}^{t} \int_{B_{\tilde{g}}\left(x_{0}, r+1\right)}\left|\nabla^{3} \phi\right|_{g}^{2} \xi^{2} d V d t+C_{14} \int_{0}^{t} \int_{B_{\tilde{g}}\left(x_{0}, r+1\right)}\left|\nabla^{2} \phi\right|_{g}^{2} d V d t,
\end{aligned}
$$

by 3.71 and the fact that $|\nabla \xi|_{g}=|\tilde{\nabla} \xi|_{g} \leq \sqrt{2}|\tilde{\nabla} \xi|_{\tilde{g}} \leq 8 \sqrt{2}$. We now estimate the rest terms on the right-hand side of (3.105). By the estimate below (3.98), we have

$$
\begin{aligned}
& \int_{B_{\tilde{g}}\left(x_{0}, r+1\right)} g^{*-4} * \operatorname{Rm} *\left(\nabla^{2} \phi\right)^{* 2} \xi^{2} d V \\
\leq & \frac{1}{4} \int_{B_{\tilde{g}}\left(x_{0}, r+1\right)}|\nabla \mathrm{Rm}|_{g}^{2} \xi^{2} d V+\epsilon \int_{B_{\tilde{g}}\left(x_{0}, r+1\right)}\left|\nabla^{3} \phi\right|_{g}^{2} \xi^{2} d V \\
& +C_{15} \int_{B_{\tilde{g}}\left(x_{0}, r+1\right)}|\nabla \tilde{\nabla} \Theta|_{g}^{2} d V,
\end{aligned}
$$

where we replaced the coefficients $1 / 2$ by $1 / 4$ (however the proof is the same). Using 3.71), $|\mathrm{Rm}|_{g}^{2} \lesssim 1+|\nabla \tilde{\nabla} g|_{g}^{2}$ (see the equation (90) in page 277 of [26]), and Lemma 3.11 the integral of $g^{*-3} *(\nabla \phi)^{* 2} *\left(\nabla^{2} \phi\right)^{* 2}+g^{*-4} * \mathrm{Rm} *(\nabla \phi)^{* 2} * \nabla^{2} \phi+g^{*-2} *\left(\nabla^{2} \phi\right)^{* 2}$ is bounded by

$$
\int_{0}^{t} \int_{B_{\tilde{g}}\left(x_{0}, r+1\right)}\left[\left|\nabla^{2} \phi\right|_{g}^{2}+|\mathrm{Rm}|_{g}^{2}\right] d V d t \lesssim \int_{0}^{t} \int_{B_{\tilde{g}}\left(x_{0}, r+1\right)}\left[1+|\nabla \tilde{\nabla} \boldsymbol{\Theta}|_{g}^{2}\right] d V d t \lesssim 1
$$

where we used the fact that $T$ depends on the given constants and the volume estimate (3.78), from the second step to the third step. By (3.71), we get

$$
\begin{aligned}
& \int_{0}^{t} \int_{B_{\tilde{g}}\left(x_{0}, r+1\right)} g^{*-3} *\left(\nabla^{2} \phi\right)^{* 3} \xi^{2} d V d t \\
= & \int_{0}^{t} \int_{B_{\tilde{g}}\left(x_{0}, r+1\right)} \nabla \phi * \nabla\left(\xi^{2} g^{*-3} *\left(\nabla^{2} \phi\right)^{* 2}\right) d V d t \\
= & \int_{0}^{t} \int_{B_{\tilde{g}}\left(x_{0}, r+1\right)} \nabla \phi *\left(\xi \nabla \xi * g^{*-3} *\left(\nabla^{2} \phi\right)^{* 2}+\xi^{2} g^{*-4} * \nabla g *\left(\nabla^{2} \phi\right)^{* 2}\right. \\
& \left.+\xi^{2} g^{*-3} * \nabla^{2} \phi * \nabla^{3} \phi\right) d V d t \\
\leq & \epsilon \int_{0}^{t} \int_{B_{\tilde{g}}\left(x_{0}, r+1\right)}\left|\nabla^{3} \phi\right|_{g}^{2} \xi^{2} d V d t+C_{16} \int_{0}^{t} \int_{B_{\tilde{g}}\left(x_{0}, r+1\right)}\left|\nabla^{2} \phi\right|_{g}^{2} d V d t ;
\end{aligned}
$$


similarly, according to the definition $V=g^{-1} * \tilde{\nabla} g$,

$$
\begin{aligned}
& \int_{0}^{t} \int_{B_{\tilde{g}}\left(x_{0}, r+1\right)} g^{*-3} * \nabla \phi * \nabla^{2} \phi * \nabla^{3} \phi \xi^{2} d V d t \\
\leq & \epsilon \int_{0}^{t} \int_{B_{\tilde{g}}\left(x_{0}, r+1\right)}\left|\nabla^{3} g\right|_{g}^{2} \xi^{2} d V d t+C_{17} \int_{0}^{t} \int_{B_{\tilde{g}}\left(x_{0}, r+1\right)}\left|\nabla^{2} g\right|_{g}^{2} d V d t, \\
& \int_{0}^{t} \int_{B_{\tilde{g}}\left(x_{0}, r+1\right)} g^{*-3} * V * \nabla^{2} \phi * \nabla^{3} \phi \xi^{2} d V d t \\
\leq & \epsilon \int_{0}^{t} \int_{B_{\tilde{g}}\left(x_{0}, r+1\right)}\left|\nabla^{3} \phi\right|_{g}^{2} \xi^{2} d V d t+C_{18} \int_{0}^{t} \int_{B_{\tilde{g}}\left(x_{0}, r+1\right)}\left|\nabla^{2} \phi\right|_{g}^{2} d V d t .
\end{aligned}
$$

Taking the integration by parts on $\nabla V$ yields

$$
\begin{aligned}
& \int_{0}^{t} \int_{B_{\tilde{g}}\left(x_{0}, r+1\right)} g^{*-3} * \nabla V *\left(\nabla^{2} \phi\right)^{* 2} \xi^{2} d V d t \\
= & \int_{0}^{t} \int_{B_{\tilde{g}}\left(x_{0}, r+1\right)} V * \nabla\left(\xi^{2} g^{*-3} *\left(\nabla^{2} \phi\right)^{* 2}\right) d V d t \\
= & \int_{0}^{t} \int_{B_{\tilde{g}}\left(x_{0}, r+1\right)} V *\left(\xi^{2} g^{*-4} * \nabla g *\left(\nabla^{2} \phi\right)^{* 2}+\xi^{2} g^{*-3} * \nabla^{2} \phi * \nabla^{3} \phi\right. \\
& \left.+g^{*-3} *\left(\nabla^{2} \phi\right)^{* 2} * \nabla \xi \xi\right) d V d t \\
\leq & \epsilon \int_{0}^{t} \int_{B_{\tilde{g}}\left(x_{0}, r+1\right)}\left|\nabla^{3} \phi\right|_{g}^{2} \xi^{2} d V d t+C_{19} \int_{0}^{t} \int_{B_{\tilde{g}}\left(x_{0}, r+1\right)}\left|\nabla^{2} \phi\right|_{g}^{2} d V d t .
\end{aligned}
$$

Substituting the above estimates into (3.105) and using Lemma 3.11, we have

$$
\begin{aligned}
\int_{B_{\tilde{g}}\left(x_{0}, r+1\right)}\left|\nabla^{2} \phi\right|_{g}^{2} \xi^{2} d V \leq & C_{20}+\frac{1}{4} \int_{0}^{t} \int_{\Omega}|\nabla \mathrm{Rm}|_{g}^{2} \xi^{2} d V d t \\
& -\left(\frac{3}{2}-5 \epsilon\right) \int_{0}^{t} \int_{B_{\tilde{g}}\left(x_{0}, r+1\right)}\left|\nabla^{3} \phi\right|_{g}^{2} \xi^{2} d V d t
\end{aligned}
$$

where $\epsilon$ is a sufficiently small positive number that shall be determined later. Combining (3.100) with 3.106), we arrive at

$$
\begin{aligned}
\int_{B_{\tilde{g}}\left(x_{0}, r+1\right)}\left[|\mathrm{Rm}|_{g}^{2}+\left|\nabla^{2} \phi\right|_{g}^{2}\right] \xi^{2} d V \leq & C_{21}-\frac{1}{4} \int_{0}^{t} \int_{B_{\tilde{g}}\left(x_{0}, r+1\right)}|\nabla \mathrm{Rm}|_{g}^{2} \xi^{2} d V d t \\
& -\left(\frac{3}{2}-5 \epsilon\right) \int_{0}^{t} \int_{B_{\tilde{g}}\left(x_{0}, r+1\right)}\left|\nabla^{3} \phi\right|_{g}^{2} \xi^{2} d V d t .
\end{aligned}
$$

As a consequence of the above estimate (3.107), we can conclude that the integral of $|\mathrm{Rm}|_{g}^{2}+\left|\nabla^{2} \phi\right|_{g}^{2}$ over the metric ball $B_{\tilde{g}}\left(x_{0}, r\right)$ is uniformly bounded from above. We here keep the minus terms on the right-hand side of (3.107) to deal with the integral of $|\nabla V|_{g}^{2} \xi^{2}$ over $B_{\tilde{g}}\left(x_{0}, r+1\right)$, and therefore, we prove Lemma 3.12

Since the metric $g$ is equivalent to $\tilde{g}$, we may write $g^{*-k} * g^{\ell}=g^{*(\ell-k)}$. Under this convenience, the equation (3.74) can be written as

$$
\begin{aligned}
\partial_{t} \nabla V= & \Delta \nabla V+g^{*-3} * \nabla \tilde{\nabla} g * \mathrm{Rm}+g^{*-3} * \tilde{\nabla} g * \nabla \mathrm{Rm}+g^{*-2} * \nabla \tilde{\nabla} g * \nabla V \\
(3.108)= & +g^{*-2} * \tilde{\nabla} g * \nabla^{2} V+g^{*-2} * \nabla \tilde{\nabla} g *(\nabla \phi)^{* 2}+g^{*-2} * \tilde{\nabla} g * \nabla \phi * \nabla^{2} \phi .
\end{aligned}
$$


From $|\nabla V|_{g}^{2}=g^{i k} g^{j \ell} \nabla_{i} V_{j} \nabla_{k} V_{\ell}$ we obtain

$$
\partial_{t}|\nabla V|_{g}^{2}=2\left\langle\nabla V, \partial_{t} \nabla V\right\rangle_{g}+g^{*-4} * \mathrm{Rm} *(\nabla V)^{* 2}+g^{*-3} *(\nabla V)^{* 3} ;
$$

by the evolution equation of $\partial_{t} g^{-1}$ above (3.73), we arrive at

$$
\begin{aligned}
\partial_{t}|\nabla V|_{g}^{2}= & 2\left\langle\nabla V, \partial_{t} \nabla V\right\rangle_{g}+g^{*-4} * \mathrm{Rm} *(\nabla V)^{* 2}+g^{*-3} *(\nabla V)^{* 3} \\
& +g^{*-3} *(\nabla V)^{* 2} *(\nabla \phi)^{* 2} .
\end{aligned}
$$

Calculate

$$
\begin{aligned}
& \int_{B_{\tilde{g}}\left(x_{0}, r+1\right)}|\nabla V|_{g}^{2} \xi^{2} d V=\int_{0}^{t}\left(\frac{d}{d t} \int_{B_{\tilde{g}}\left(x_{0}, r+1\right)}|\nabla V|_{g}^{2} \xi^{2} d V\right) d t \\
= & \int_{0}^{t} \int_{B_{\tilde{g}\left(x_{0}, r+1\right)}}|\nabla V|^{2} \xi^{2} \partial_{t} d V d t+\int_{0}^{t} \int_{B_{\tilde{g}}\left(x_{0}, r+1\right)} \partial_{t}|\nabla V|_{g}^{2} \xi^{2} d V d t .
\end{aligned}
$$

since $V=0$ at $t=0$. Plugging (3.108, 3.109) into 3.100, we get

$$
\begin{aligned}
& \int_{B_{\tilde{g}}\left(x_{0}, r+1\right)}|\nabla V|_{g}^{2} \xi^{2} d V \\
= & 2 \int_{0}^{t} \int_{B_{\tilde{g}}\left(x_{0}, r+1\right)}\langle\nabla V, \Delta \nabla V\rangle_{g} \xi^{2} d V d t \\
& +\int_{0}^{t} \int_{B_{\tilde{g}}\left(x_{0}, r+1\right)}\left[g^{*-5} * \nabla \tilde{\nabla} g * \mathrm{Rm} * \nabla V+g^{*-5} * \tilde{\nabla} g * \nabla \mathrm{Rm} * \nabla V\right. \\
(3.111)+ & g^{*-4} * \nabla \tilde{\nabla} g *(\nabla V)^{* 2}+g^{*-4} * \tilde{\nabla} g * \nabla V * \nabla^{2} V+g^{*-4} * \mathrm{Rm} *(\nabla V)^{* 2} \\
& +g^{*-3} *(\nabla V)^{* 3}+g^{*-4} * \nabla \tilde{\nabla} g * \nabla V *(\nabla \phi)^{* 2} \\
& \left.+g^{*-4} * \tilde{\nabla} g * \nabla V * \nabla \phi * \nabla^{2} \phi+g^{*-3} *(\nabla V)^{* 2} *(\nabla \phi)^{* 2}\right] \xi^{2} d V d t .
\end{aligned}
$$

The first term on the right-hand side of (3.111) was computed in [26] ( see the equation (104) in page 280):

$$
\begin{aligned}
2 \int_{0}^{t} \int_{B_{\tilde{g}}\left(x_{0}, r+1\right)}\langle\nabla V, \Delta \nabla V\rangle_{g} \xi^{2} d V d t \leq & -\frac{15}{8} \int_{0}^{t} \int_{B_{\tilde{g}}\left(x_{0}, r+1\right)}\left|\nabla^{2} V\right|_{g}^{2} \xi^{2} d V d t \\
& +C_{22} \int_{0}^{t} \int_{B_{\tilde{g}}\left(x_{0}, r+1\right)}|\nabla V|_{g}^{2} d V d t .
\end{aligned}
$$

Define

$$
\begin{aligned}
I_{1}:= & g^{*-5} * \tilde{\nabla} g * \nabla \operatorname{Rm} * \nabla V+g^{*-4} * \tilde{\nabla} g * \nabla V * \nabla^{2} V, \\
I_{2}:= & g^{*-5} * \nabla \tilde{\nabla} g * \operatorname{Rm} * \nabla V, \\
I_{3}:= & g^{*-4} * \nabla \tilde{\nabla} g *(\nabla V)^{* 2}+g^{*-4} * \operatorname{Rm} *(\nabla V)^{* 2}+g^{*-3} *(\nabla V)^{* 3}, \\
I_{4}:= & g^{*-4} * \nabla \tilde{\nabla} g * \nabla V *(\nabla \phi)^{* 2}+g^{*-4} * \tilde{\nabla} g * \nabla V * \nabla \phi * \nabla^{2} \phi \\
& +g^{*-3} *(\nabla V)^{* 2} *(\nabla \phi)^{* 2} .
\end{aligned}
$$

According to (106) in page 280 of [26], we have

$$
\begin{aligned}
\int_{0}^{t} \int_{B_{\tilde{g}}\left(x_{0}, r+1\right)} I_{1} \xi^{2} d V d t \leq & \frac{1}{16} \int_{0}^{t} \int_{B_{\tilde{g}}\left(x_{0}, r+1\right)}\left[|\nabla \mathrm{Rm}|_{g}^{2}+\left|\nabla^{2} V\right|_{g}^{2}\right] \xi^{2} d V d t \\
& +C_{23} \int_{0}^{t} \int_{B_{\tilde{g}}\left(x_{0}, r+1\right)}|\nabla V|_{g}^{2} d V d t
\end{aligned}
$$


according to (107) in page 280 and (112) in page 281 of [26], we have

$$
\begin{aligned}
\int_{0}^{t} \int_{B_{\tilde{g}}\left(x_{0}, r+1\right)} I_{2} \xi^{2} d V d t \leq & \frac{1}{16} \int_{0}^{t} \int_{B_{\tilde{g}}\left(x_{0}, r+1\right)}\left[|\nabla \mathrm{Rm}|_{g}^{2}+\left|\nabla^{2} V\right|_{g}^{2}\right] \xi^{2} d V d t \\
& +C_{24} \int_{0}^{t} \int_{B_{\tilde{g}}\left(x_{0}, r+1\right)}\left[|\mathrm{Rm}|_{g}^{2}+|\nabla V|_{g}^{2}\right] d V d t, \\
\int_{0}^{t} \int_{B_{\tilde{g}}\left(x_{0}, r+1\right)} I_{3} \xi^{2} d V d t \leq & \frac{1}{8} \int_{0}^{t} \int_{B_{\tilde{g}}\left(x_{0}, r+1\right)}\left|\nabla^{2} V\right|_{g}^{2} \xi^{2} d V d t \\
& +C_{25} \int_{0}^{t} \int_{B_{\tilde{g}}\left(x_{0}, r+1\right)}|\nabla V|_{g}^{2} \xi^{2} d V d t .
\end{aligned}
$$

Using 3.71 implies

$$
\begin{aligned}
& \int_{0}^{t} \int_{B_{\tilde{g}}\left(x_{0}, r+1\right)} I_{4} \xi^{2} d V d t \\
\lesssim & \int_{0}^{t} \int_{B_{\tilde{g}}\left(x_{0}, r+1\right)}\left(|\nabla \tilde{\nabla} g|_{g}^{2}+|\nabla V|_{g}^{2}+\left|\nabla^{2} \phi\right|_{g}^{2}\right) \xi^{2} d V d t .
\end{aligned}
$$

Substituting 3.112, 3.113, 3.114, 3.115, 3.116 into 3.111, using the fact that $\nabla V=g^{-1} * \nabla \tilde{\nabla} g$, and using Lemma 3.11] we obtain

$$
\begin{aligned}
\int_{B_{\tilde{g}}\left(x_{0}, r+1\right)}|\nabla V|_{g}^{2} \xi^{2} d V_{t} \leq & -\frac{13}{8} \int_{0}^{t} \int_{B_{\tilde{g}}\left(x_{0}, r+1\right)}\left|\nabla^{2} V\right|_{g}^{2} \xi^{2} d V d t \\
& +\frac{1}{8} \int_{0}^{t} \int_{B_{\tilde{g}}\left(x_{0}, r+1\right)}|\nabla \mathrm{Rm}|_{g}^{2} \xi^{2} d V d t+C_{26} .
\end{aligned}
$$

Choosing $\epsilon=11 / 40$ in 3.107) and combining with 3.117), we arrive at

$$
\begin{aligned}
& \int_{B_{\tilde{g}}\left(x_{0}, r+1\right)}\left[|\mathrm{Rm}|_{g}^{2}+\left|\nabla^{2} \phi\right|_{g}^{2}+|\nabla V|_{g}^{2}\right] \xi^{2} d V \\
& +\frac{1}{8} \int_{0}^{t} \int_{B_{\tilde{g}}\left(x_{0}, r+1\right)}\left[|\nabla \mathrm{Rm}|_{g}^{2}+\left|\nabla^{3} \phi\right|_{g}^{2}+\left|\nabla^{2} V\right|_{g}^{2}\right] \xi^{2} d V d t \lesssim 1 .
\end{aligned}
$$

In particular,

$$
\begin{gathered}
\max _{t \in[0, T]} \int_{B_{\tilde{g}}\left(x_{0}, r\right)}\left[|\mathrm{Rm}|_{g}^{2}+\left|\nabla^{2} \phi\right|_{g}^{2}+|\nabla V|_{g}^{2}\right] d V \lesssim 1 \\
\int_{0}^{T} \int_{B_{\tilde{g}\left(x_{0}, r\right)}}\left[|\nabla \mathrm{Rm}|_{g}^{2}+\left|\nabla^{3} \phi\right|_{g}^{2}+\left|\nabla^{2} V\right|_{g}^{2}\right] d V d t \lesssim 1
\end{gathered}
$$

since $\xi \equiv 1$ on $B_{\tilde{g}}\left(x_{0}, r\right)$.

Recall that

$$
\partial_{t} \mathrm{Rm}=\Delta \mathrm{Rm}+g^{*-2} * \mathrm{Rm}^{* 2}+g^{-1} * V * \nabla \mathrm{Rm}+g^{-1} * \mathrm{Rm} * \nabla V+\left(\nabla^{2} \phi\right)^{* 2} .
$$

Since $\nabla\left(g^{-1} * V * \mathrm{Rm}\right)=g^{-1} * V * \nabla \mathrm{Rm}+g^{-1} * \mathrm{Rm} * \nabla V$, it follows that

$$
\partial_{t} \mathrm{Rm}=\Delta \mathrm{Rm}+\nabla P_{1}+Q_{1}
$$

where

$$
P_{1}:=g^{-1} * V * \mathrm{Rm}, \quad Q_{1}:=g^{-1} * \mathrm{Rm} * \nabla V+g^{*-2} * \mathrm{Rm}^{* 2}+\left(\nabla^{2} \phi\right)^{* 2} .
$$


Recall the equation

$$
\partial_{t} \nabla V=\nabla\left(\partial_{t} V\right)+V * \partial_{t} \Gamma, \quad \partial_{t} \Gamma=g^{-1} * \nabla\left(\partial_{t} g\right)
$$

after 3.73 and the equation $\partial_{t} g=g^{-1} * \mathrm{Rm}+\nabla V+(\nabla \phi)^{* 2}$. Hence

$$
\begin{aligned}
\partial_{t} \nabla V= & \nabla\left(\Delta V+g^{*-3} * \tilde{\nabla} g * \mathrm{Rm}+g^{*-2} * \tilde{\nabla} g * \nabla V+g^{*-2} * \tilde{\nabla} g *(\nabla \phi)^{* 2}\right) \\
& +g^{*-2} * V * \nabla \mathrm{Rm}+g^{-1} * V * \nabla^{2} V+g^{-1} * V * \nabla \phi * \nabla^{2} \phi .
\end{aligned}
$$

From the Ricci identity $\nabla \Delta V=\Delta \nabla V+g^{*-2} * \mathrm{Rm} * \nabla V+g^{*-2} * V * \nabla \mathrm{Rm}$, it follows that

$$
\begin{aligned}
\partial_{t} \nabla V= & \Delta \nabla V+\nabla\left(g^{*-3} * \tilde{\nabla} g * \mathrm{Rm}+g^{*-2} * \tilde{\nabla} g * \nabla V+g^{*-2} * \tilde{\nabla} g *(\nabla \phi)^{* 2}\right) \\
& +g^{*-2} * V * \nabla \mathrm{Rm}+g^{-1} * V * \nabla^{2} V+g^{*-2} * \mathrm{Rm} * \nabla V \\
& +g^{-1} * V * \nabla \phi * \nabla^{2} \phi .
\end{aligned}
$$

Since

$$
\begin{aligned}
\nabla\left(g^{*-2} * V * \mathrm{Rm}\right) & =g^{*-2} * V * \nabla \mathrm{Rm}+g^{*-2} * \mathrm{Rm} * \nabla V \\
\nabla\left(g^{-1} * V * \nabla V\right) & =g^{-1} * V * \nabla^{2} V+g^{-1} *(\nabla V)^{* 2}
\end{aligned}
$$

and $V=g^{-1} * \tilde{\nabla} g$, we obtain

$$
\begin{aligned}
g^{*-2} * V * \nabla \mathrm{Rm} & =\nabla\left(g^{*-3} * \tilde{\nabla} g * \mathrm{Rm}\right)+g^{*-2} * \mathrm{Rm} * \nabla V, \\
g^{-1} * V * \nabla^{2} V & =\nabla\left(g^{*-2} * \tilde{\nabla} g * \nabla V\right)+g^{-1} *(\nabla V)^{* 2}
\end{aligned}
$$

and hence

$$
\partial_{t} \nabla V=\Delta \nabla V+\nabla P_{2}+Q_{2}
$$

where

$$
\begin{aligned}
P_{2} & =g^{*-3} * \tilde{\nabla} g * \mathrm{Rm}+g^{*-2} * \tilde{\nabla} g * \nabla V+g^{*-2} * \tilde{\nabla} g *(\nabla \phi)^{* 2}, \\
Q_{2} & =g^{*-2} * \mathrm{Rm} * \nabla V+g^{-1} *(\nabla V)^{* 2}+g^{-1} * V * \nabla \phi * \nabla^{2} \phi .
\end{aligned}
$$

Finally, according to 3.75, we have

$$
\partial_{t} \nabla^{2} \phi=\Delta \nabla^{2} \phi+\nabla P_{3}+Q_{3}
$$

where

$$
\begin{aligned}
P_{3} & =g^{-1} * \nabla \phi * \nabla^{2} \phi+g^{-1} * V * \nabla^{2} \phi, \\
Q_{3} & =g^{*-2} * \operatorname{Rm} * \nabla^{2} \phi+g^{-1} *(\nabla \phi)^{* 2} * \nabla^{2} \phi+g^{*-2} * \operatorname{Rm} *(\nabla \phi)^{* 2}+\beta_{2} \nabla^{2} \phi .
\end{aligned}
$$

Lemma 3.13. For any integer $n \geq 1$, we have

$$
\begin{aligned}
\int_{0}^{T} \int_{B_{\tilde{g}}\left(x_{0}, r\right)} u^{n-1}|\nabla \tilde{\nabla} g|_{g}^{2} d V d t & \lesssim 1, \\
\max _{t \in[0, T]} \int_{B_{\tilde{g}}\left(x_{0}, r\right)} u^{n} d V & \lesssim 1, \\
\int_{0}^{T} \int_{B_{\tilde{g}}\left(x_{0}, r\right)} u^{n-1} v d V d t & \lesssim 1 .
\end{aligned}
$$

where

$$
u:=|\mathrm{Rm}|_{g}^{2}+\left|\nabla^{2} \phi\right|_{g}^{2}+|\nabla V|_{g}^{2}, \quad v:=|\nabla \mathrm{Rm}|_{g}^{2}+\left|\nabla^{3} \phi\right|_{g}^{2}+\left|\nabla^{2} V\right|_{g}^{2},
$$

and $\lesssim$ depends on $m, n, r, k_{0}, k_{1}, k_{2}, \alpha_{1}, \beta_{1}, \beta_{2}$. 
Proof. The case $n=1$ follows from Lemma 3.11 and Lemma 3.12 . We now prove by induction on $n$. Suppose that for $s=1, \cdots, n-1$, we have

$$
\int_{0}^{T} \int_{B_{\tilde{g}}\left(x_{0}, r\right)} u^{s-1}|\nabla \tilde{\nabla} g|_{g}^{2} d V d t, \max _{t \in[0, T]} \int_{B_{\tilde{g}}\left(x_{0}, r\right)} u^{s} d V, \int_{0}^{T} \int_{B_{\tilde{g}}\left(x_{0}, r\right)} u^{s-1} v d V d t \lesssim 1 .
$$

For convenience, define

$$
w:=|\operatorname{Rm}|_{\tilde{g}}^{2}+\left|\nabla^{2} \phi\right|_{\tilde{g}}^{2}+|\nabla V|_{\tilde{g}}^{2} .
$$

By (66) in page 272 of [26] and 3.71), we have $|\nabla \tilde{\nabla} g|_{g}^{2} \leq 2\left|\tilde{\nabla}^{2} g\right|_{g}^{2}+C_{1} \leq 32\left|\tilde{\nabla}^{2} g\right|_{\tilde{g}}^{2}+$ $C_{1}$ and hence

$$
\int_{0}^{T} \int_{B_{\tilde{g}}\left(x_{0}, r+1\right)} u^{n-1}|\nabla \tilde{\nabla} g|_{g}^{2} d V d t \leq 32 \int_{0}^{T} \int_{B_{\tilde{g}}\left(x_{0}, r+1\right)} u^{n-1}\left|\tilde{\nabla}^{2} g\right|_{\tilde{g}}^{2} d V d t+C_{2}
$$

by 3.78 and 3.71 . Since $\frac{1}{16} w \leq u \leq 16 w$, to estimate

$$
\int_{0}^{T} \int_{B_{\tilde{g}}\left(x_{0}, r+1\right)} u^{n-1}|\nabla \tilde{\nabla} g|_{g}^{2} d V d t
$$

we suffice to estimate

$$
\int_{0}^{T} \int_{B_{\tilde{g}}\left(x_{0}, r+1\right)} w^{n-1}\left|\tilde{\nabla}^{2} g\right|_{\tilde{g}}^{2} d \tilde{V} d t
$$

since $d V \leq 2^{m / 2} d \tilde{V}$. Consider the same cutoff function $\xi(x)$ used in the proof of Lemma 3.10. Calculate

$$
\begin{aligned}
K:= & \frac{d}{d t} \int_{B_{\tilde{g}}\left(x_{0}, r+1\right)} w^{n-1}|\tilde{\nabla} g|_{\tilde{g}}^{2} \xi^{2} d \tilde{V} \\
= & \int_{B_{\tilde{g}}\left(x_{0}, r+1\right)} w^{n-1} 2\left\langle\tilde{\nabla} g, \partial_{t} \tilde{\nabla} g\right\rangle_{\tilde{g}} \xi^{2} d \tilde{V} \\
& +\int_{B_{\tilde{g}}\left(x_{0}, r+1\right)}|\tilde{\nabla} g|_{\tilde{g}}^{2}(n-1) w^{n-2}\left[\partial_{t}|\operatorname{Rm}|_{\tilde{g}}^{2}+\partial_{t}\left|\nabla^{2} \phi\right|_{\tilde{g}}^{2}+\partial_{t}|\nabla V|_{\tilde{g}}^{2}\right] \xi^{2} d \tilde{V} \\
:= & I+J .
\end{aligned}
$$

Using the evolution equation of $\tilde{\nabla} g$ after 3.77 yields

$$
\begin{aligned}
I= & 2 \int_{B_{\tilde{g}}\left(x_{0}, r+1\right)} \xi^{2} w^{n-1}\left\langle\tilde{\nabla} g, g^{\alpha \beta} \tilde{\nabla}_{\alpha} \tilde{\nabla}{ }_{\beta} \tilde{\nabla} g+g^{-1} * g * \tilde{\nabla} \widetilde{\mathrm{Rm}}+\tilde{\nabla} \phi * \tilde{\nabla}^{2} \phi\right. \\
& \left.+g^{*-2} * g * \tilde{\nabla} g * \widetilde{\mathrm{Rm}}+g^{*-2} * \tilde{\nabla} g * \tilde{\nabla}^{2} g+g^{*-3} *(\tilde{\nabla} g)^{* 3}\right\rangle_{\tilde{g}} d \tilde{V} \\
\leq & C_{3} \int_{B_{\tilde{g}}\left(x_{0}, r+1\right)}\left(1+\left|\tilde{\nabla}^{2} g\right|_{\tilde{g}}\right) w^{n-1} \xi^{2} d \tilde{V}+I_{1}+I_{2}+I_{3}
\end{aligned}
$$

by (3.71), where

$$
\begin{aligned}
I_{1} & =2 \int_{B_{\tilde{g}}\left(x_{0}, r+1\right)} \xi^{2} w^{n-1}\left\langle\tilde{\nabla} g, g^{\alpha \beta} \tilde{\nabla}_{\alpha} \tilde{\nabla}_{\beta} \tilde{\nabla} g\right\rangle_{\tilde{g}} d \tilde{V} \\
I_{2} & =\int_{B_{\tilde{g}}\left(x_{0}, r+1\right)} \xi^{2} w^{n-1} * g^{-1} * g * \tilde{\nabla} g * \tilde{\nabla} \widetilde{\operatorname{Rm}} d \tilde{V} \\
I_{3} & =\int_{B_{\tilde{g}}\left(x_{0}, r+1\right)} \xi^{2} w^{n-1} * \tilde{\nabla} g * \tilde{\nabla} \phi * \tilde{\nabla}^{2} \phi d \tilde{V}
\end{aligned}
$$


By integration by parts, the term $I_{1}$ can be estimated by

$$
\begin{aligned}
I_{1}= & -2 \int_{B_{\tilde{g}}\left(x_{0}, r+1\right)}\left\langle\tilde{\nabla}_{\beta} \tilde{\nabla} g, \tilde{\nabla}_{\alpha}\left(g^{\alpha \beta} \xi^{2} w^{n-1} \tilde{\nabla} g\right)\right\rangle_{\tilde{g}} d \tilde{V} \\
= & -2 \int_{B_{\tilde{g}}\left(x_{0}, r+1\right)}\left\langle\tilde{\nabla}_{\beta} \tilde{\nabla} g, g^{\alpha \beta} \xi^{2} w^{n-1} \tilde{\nabla}_{\alpha} \tilde{\nabla} g\right. \\
& +g^{*-2} *(\tilde{\nabla} g)^{* 2} * \xi^{2} * w^{n-1}+g^{-1} * \xi * \tilde{\nabla} \xi * \tilde{\nabla} g * w^{n-1} \\
& \left.+g^{-1} * \tilde{\nabla} g * \xi^{2} w^{n-2} *\left(\mathrm{Rm} * \tilde{\nabla} \mathrm{Rm}+\nabla V * \tilde{\nabla} \nabla V+\nabla^{2} \phi * \tilde{\nabla} \nabla^{2} \phi\right)\right\rangle_{\tilde{g}} d \tilde{V} \\
\leq & \int_{B_{\tilde{g}}\left(x_{0}, r+1\right)}\left[-\left|\tilde{\nabla}^{2} g\right|_{\tilde{g}}^{2} \xi^{2} w^{n-1}+C_{4}\left|\tilde{\nabla}^{2} g\right|_{\tilde{g}} \xi w^{n-1}+\xi^{2} w^{n-2} * g^{-1} * \tilde{\nabla} g\right. \\
& \left.* \tilde{\nabla}^{2} g *\left(\mathrm{Rm} * \tilde{\nabla} \mathrm{Rm}+\nabla V * \tilde{\nabla} \nabla V+\nabla^{2} \phi * \tilde{\nabla} \nabla^{2} \phi\right)\right] d \tilde{V}
\end{aligned}
$$

by (3.71). The Cauchy-Schwarz inequality implies

$$
\begin{aligned}
& C_{4} \int_{B_{\tilde{g}}\left(x_{0}, r+1\right)}\left|\tilde{\nabla}^{2} g\right|_{\tilde{g}} \xi w^{n-1} d \tilde{V}=C_{4} \int_{B_{\tilde{g}}\left(x_{0}, r+1\right)}\left(\left|\tilde{\nabla}^{2} g\right|_{\tilde{g}} w^{\frac{n-1}{2}} \xi\right) w^{\frac{n-1}{2}} d \tilde{V} \\
& \leq \frac{1}{8} \int_{B_{\tilde{g}\left(x_{0}, r+1\right)}}\left|\tilde{\nabla}^{2} g\right|_{\tilde{g}}^{2} \xi^{2} w^{n-1} d \tilde{V}+2 C_{4}^{2} \int_{B_{\tilde{g}}\left(x_{0}, r+1\right)} w^{n-1} d \tilde{V} \\
& \leq \frac{1}{8} \int_{B_{\tilde{g}}\left(x_{0}, r+1\right)}\left|\tilde{\nabla}^{2} g\right|_{\tilde{g}}^{2} \xi^{2} w^{n-1} d \tilde{V}+C_{5}
\end{aligned}
$$

by the inductive hypothesis and $\frac{1}{16} w \leq u \leq 16 w$. Similarly,

$$
\begin{aligned}
& \int_{B_{\tilde{g}}\left(x_{0}, r+1\right)} \xi^{2} w^{n-2} * g^{-1} * \tilde{\nabla} g * \tilde{\nabla}^{2} g *(\mathrm{Rm} * \tilde{\nabla} \mathrm{Rm}+\nabla V * \tilde{\nabla} \nabla V \\
& \left.+\nabla^{2} \phi * \tilde{\nabla} \nabla^{2} \phi\right) d \tilde{V} \\
\leq & C_{6} \int_{B_{\tilde{g}}\left(x_{0}, r+1\right)}\left(w^{\frac{n-2}{2}} \xi\left|\tilde{\nabla}^{2} g\right|_{\tilde{g}}|\mathrm{Rm}| \tilde{g}\right)\left(w^{\frac{n-2}{2}} \xi|\tilde{\nabla} \mathrm{Rm}|_{\tilde{g}}\right) d \tilde{V} \\
& +C_{6} \int_{B_{\tilde{g}\left(x_{0}, r+1\right)}}\left(w^{\frac{n-2}{2}} \xi\left|\tilde{\nabla}^{2} g\right|_{\tilde{g}}|\nabla V|_{\tilde{g}}\right)\left(w^{\frac{n-2}{2}} \xi|\tilde{\nabla} \nabla V|_{\tilde{g}}\right) d \tilde{V} \\
& +C_{6} \int_{B_{\tilde{g}\left(x_{0}, r+1\right)}}\left(w^{\frac{n-2}{2}} \xi\left|\tilde{\nabla}^{2} g\right|_{\tilde{g}}\left|\nabla^{2} \phi\right|_{\tilde{g}}\right)\left(w^{\frac{n-2}{2}} \xi\left|\tilde{\nabla} \nabla^{2} \phi\right|_{\tilde{g}}\right) d \tilde{V} \\
\leq & \frac{1}{8} \int_{B_{\tilde{g}}\left(x_{0}, r+1\right)}\left|\tilde{\nabla}^{2} g\right|_{\tilde{g}}^{2} w^{n-1} \xi^{2} d \tilde{V} \\
& +C_{7} \int_{B_{\tilde{g}}\left(x_{0}, r+1\right)} w^{n-2} \xi^{2}\left(|\tilde{\nabla} \mathrm{Rm}|_{\tilde{g}}^{2}+|\tilde{\nabla} \nabla V|_{\tilde{g}}^{2}+\left|\tilde{\nabla} \nabla^{2} \phi\right|_{\tilde{g}}^{2}\right) d \tilde{V} .
\end{aligned}
$$

According to $\Gamma-\tilde{\Gamma}=g^{-1} * \tilde{\nabla} g$, we have

$$
\begin{aligned}
\tilde{\nabla} \mathrm{Rm} & =\nabla \mathrm{Rm}+g^{-1} * \tilde{\nabla} g * \mathrm{Rm}, \quad \tilde{\nabla} \nabla V=\nabla^{2} V+g^{-1} * \tilde{\nabla} g * \nabla V, \\
\tilde{\nabla} \nabla^{2} \phi & =\nabla^{3} \phi+g^{-1} * \tilde{\nabla} g * \nabla^{2} \phi,
\end{aligned}
$$


from which we can conclude that $|\tilde{\nabla} \mathrm{Rm}|_{\tilde{g}}^{2}+|\tilde{\nabla} \nabla V|_{\tilde{g}}^{2}+\left|\tilde{\nabla} \nabla^{2} \phi\right|_{\tilde{g}}^{2} \lesssim u+v$ and then

$$
\begin{aligned}
& \int_{B_{\tilde{g}}\left(x_{0}, r+1\right)} \xi^{2} w^{n-2} * g^{-1} * \tilde{\nabla} g * \tilde{\nabla}^{2} g *(\mathrm{Rm} * \tilde{\nabla} \mathrm{Rm}+\nabla V * \tilde{\nabla} \nabla V \\
& \left.+\left|\tilde{\nabla} \nabla^{2} \phi\right|_{\tilde{g}}^{2}\right) d \tilde{V} \\
\leq & \frac{1}{8} \int_{B_{\tilde{g}}\left(x_{0}, r+1\right)}\left|\tilde{\nabla}^{2} g\right|_{\tilde{g}}^{2} w^{n-1} \xi^{2} d \tilde{V}+C_{8} \int_{B_{\tilde{g}}\left(z_{0}, r+1\right)} w^{n-2} \xi^{2}(u+v) d \tilde{V} \\
\leq & \frac{1}{8} \int_{B_{\tilde{g}}\left(x_{0}, r+1\right)}|\tilde{\nabla} g|_{\tilde{g}}^{2} w^{n-1} \xi^{2} d \tilde{V}+C_{9} \int_{B_{\tilde{g}}\left(x_{0}, r+1\right)} u^{n-2} \xi^{2}(u+v) d \tilde{V} \\
\leq & \frac{1}{8} \int_{B_{\tilde{g}}\left(x_{0}, r+1\right)}|\tilde{\nabla} g|_{\tilde{g}}^{2} w^{n-1} \xi^{2} d \tilde{V}+C_{9} \int_{B_{\tilde{g}}\left(x_{0}, r+1\right)} u^{n-2} v \xi^{2} d \tilde{V}+C_{10}
\end{aligned}
$$

by the inductive hypothesis. Consequently,

$$
I_{1} \leq-\frac{3}{4} \int_{B_{\tilde{g}}\left(x_{0}, r+1\right)}|\tilde{\nabla} g|_{\tilde{g}}^{2} w^{n-1} \xi^{2} d \tilde{V}+C_{11} \int_{B_{\tilde{g}}\left(x_{0}, r+1\right)} u^{n-2} v d \tilde{V}+C_{12}
$$

If we directly use the inequalities (3.71), we can get the uniform upper bound for $I_{2}$ by the inductive hypothesis. However, in this case the bound shall depend on an upper bound of $|\tilde{\nabla} \widehat{\mathrm{Rm}}|_{\tilde{g}}$. To fund the dependence of $k_{0}$, we will argue as follows. Again using the integration by parts, we get

$$
\begin{aligned}
I_{2}= & -\int_{B_{\tilde{g}}\left(x_{0}, r+1\right)}\left\langle\widetilde{\mathrm{Rm}}, \tilde{\nabla}\left(g^{-1} * g * \tilde{\nabla} g * \xi^{2} * w^{n-1}\right)\right\rangle_{\tilde{g}} d \tilde{V} \\
= & \int_{B_{\tilde{g}}\left(x_{0}, r+1\right)}\left\langle\widetilde{\mathrm{Rm}}, g^{*-2} *(\tilde{\nabla} g)^{* 2} * g * \xi^{2} * w^{n-1}+\left((\tilde{\nabla} g)^{* 2}+g * \tilde{\nabla}^{2} g\right)\right. \\
& * g^{-1} * \xi^{2} * w^{n-1}+g^{-1} * g * \tilde{\nabla} g * \xi * \tilde{\nabla} \xi * w^{n-1}+g^{-1} * g * \tilde{\nabla} g * \xi^{2} \\
(3.122) \quad & \left.* w^{n-2} *\left(\operatorname{Rm} * \tilde{\nabla} \mathrm{Rm}+\nabla V * \tilde{\nabla} \nabla V+\nabla^{2} \phi * \tilde{\nabla} \nabla^{2} \phi\right)\right\rangle_{\tilde{g}} d \tilde{V} \\
\leq & C_{13}+C_{13} \int_{B_{\tilde{g}}\left(x_{0}, r+1\right)} w^{n-1}\left|\tilde{\nabla}^{2} g\right|_{\tilde{g}} \xi^{2} d \tilde{V}+C_{13} \int_{B_{\tilde{g}}\left(x_{0}, r+1\right)} w^{n-2}\left(|\mathrm{Rm}|_{\tilde{g}}^{2}\right. \\
& \left.+|\nabla V|_{\tilde{g}}^{2}+|\tilde{\nabla} \mathrm{Rm}|_{\tilde{g}}^{2}+|\tilde{\nabla} \nabla V|_{\tilde{g}}^{2}+\left|\nabla^{2} \phi\right|_{\tilde{g}}^{2}+\left|\tilde{\nabla} \nabla^{2} \phi\right|_{\tilde{g}}^{2}\right) d \tilde{V} \\
\leq & \frac{1}{8} \int_{B_{\tilde{g}}\left(x_{0}, r+1\right)} w^{n-1}\left|\tilde{\nabla}^{2} g\right|_{\tilde{g}}^{2} \xi^{2} d \tilde{V}+C_{14} \int_{B_{\tilde{g}}\left(x_{0}, r+1\right)} w^{n-2}(u+v) d \tilde{V}+C_{14} \\
\leq & \frac{1}{8} \int_{B_{\tilde{g}}\left(x_{0}, r+1\right)} w^{n-1}\left|\tilde{\nabla}^{2} g\right|_{\tilde{g}}^{2} \xi^{2} d \tilde{V}+C_{15} \int_{B_{\tilde{g}}\left(x_{0}, r+1\right)} u^{n-2} v d \tilde{V}+C_{15}
\end{aligned}
$$

by the inductive hypothesis, (3.71) and the previous estimates. From (3.71), we also have

$$
I_{3} \lesssim \int_{B_{\tilde{g}}\left(x_{0}, r+1\right)} w^{n-1}\left|\tilde{\nabla}^{2} \phi\right| \tilde{g} \xi d \tilde{V}
$$


Since $C_{3}\left|\tilde{\nabla}^{2} g\right|_{\tilde{g}} \leq \frac{1}{8}\left|\tilde{\nabla}^{2} g\right|_{\tilde{g}}^{2}+2 C_{3}^{2}$, we infer from 3.121, 3.122 , and 3.123 that

$$
\begin{aligned}
I \leq & -\frac{1}{2} \int_{B_{\tilde{g}}\left(x_{0}, r+1\right)}\left|\tilde{\nabla}^{2} g\right|_{\tilde{g}}^{2} w^{n-1} \xi^{2} d \tilde{V}+C_{16} \int_{B_{\tilde{g}}\left(x_{0}, r+1\right)} u^{n-2} v d \tilde{V} \\
& +C_{16} \int_{B_{\tilde{g}}\left(x_{0}, r+1\right)} w^{n-1}\left|\tilde{\nabla}^{2} \phi\right| \tilde{g} \xi d \tilde{V}+C_{16}
\end{aligned}
$$

by the inductive hypothesis. Note that the estimate 3.82 is a special case of 3.124. According to 3.71,

$$
\begin{aligned}
J \leq & C_{17} \int_{B_{\tilde{g}}\left(x_{0}, r+1\right)} w^{n-2}\left[2\left\langle\mathrm{Rm}, \partial_{t} \mathrm{Rm}\right\rangle_{\tilde{g}}+2\left\langle\nabla^{2} \phi, \partial_{t} \nabla^{2} \phi\right\rangle_{\tilde{g}}\right. \\
& \left.+2\left\langle\nabla V, \partial_{t} \nabla V\right\rangle_{\tilde{g}}\right] \xi^{2} d \tilde{V}:=\quad C_{17}\left(J_{1}+J_{2}+J_{3}\right),
\end{aligned}
$$

where

$$
\begin{aligned}
& J_{1}:=\int_{B_{\tilde{g}}\left(x_{0}, r+1\right)} w^{n-2} 2\left\langle\mathrm{Rm}, \partial_{t} \mathrm{Rm}\right\rangle_{\tilde{g}} \xi^{2} d \tilde{V}, \\
& J_{2}:=\int_{B_{\tilde{g}}\left(x_{0}, r+1\right)} w^{n-2} 2\left\langle\nabla V, \partial_{t} \nabla V\right\rangle_{\tilde{g}} \xi^{2} d \tilde{V}, \\
& J_{3}:=\int_{B_{\tilde{g}}\left(x_{0}, r+1\right)} w^{n-2} 2\left\langle\nabla^{2} \phi, \partial_{t} \nabla^{2} \phi\right\rangle_{\tilde{g}} \xi^{2} d \tilde{V} .
\end{aligned}
$$

Substituting 3.72 into $J_{1}$ we find that

$$
\begin{aligned}
J_{1}= & \int_{B_{\tilde{g}}\left(x_{0}, r+1\right)} w^{n-2} \xi^{2} 2\left\langle\mathrm{Rm}, \Delta \mathrm{Rm}+g^{*-2} * \mathrm{Rm}^{* 2}\right. \\
& \left.+g^{-1} * V * \nabla \mathrm{Rm}+g^{-1} * \mathrm{Rm} * \nabla V+\left(\nabla^{2} \phi\right)^{* 2}\right\rangle_{\tilde{g}} d \tilde{V} .
\end{aligned}
$$

We now estimate each term in 3.125. Since $\Delta \mathrm{Rm}=g^{\alpha \beta} \nabla_{\alpha} \nabla_{\beta} \mathrm{Rm}$, the first term on the right-hand side of $(3.125)$ is bounded by

$$
\begin{aligned}
& -2 \int_{B_{\tilde{g}}\left(x_{0}, r+1\right)}\left\langle\nabla_{\beta} \mathrm{Rm}, \nabla_{\alpha}\left(\xi^{2} w^{n-2} g^{\alpha \beta} \mathrm{Rm}\right)\right\rangle_{\tilde{g}} d \tilde{V} \\
= & -2 \int_{B_{\tilde{g}}\left(x_{0}, r+1\right)}\left\langle\nabla_{\beta} \mathrm{Rm}, \xi^{2} w^{n-2} g^{\alpha \beta} \nabla_{\alpha} \operatorname{Rm}\right\rangle_{\tilde{g}} d \tilde{V} \\
& +\int_{B_{\tilde{g}}\left(x_{0}, r+1\right)} \nabla \mathrm{Rm} *\left(g^{*-2} * \nabla g * \operatorname{Rm} * \xi^{2}+g^{-1} * \mathrm{Rm} * \xi * \nabla \xi\right) w^{n-2} d \tilde{V} \\
& +\int_{B_{\tilde{g}}\left(x_{0}, r+1\right)} \nabla \mathrm{Rm} * g^{-1} * \mathrm{Rm} * \xi^{2} * w^{n-3} *\left(\mathrm{Rm} * \tilde{\nabla} \mathrm{Rm}+\nabla V * \nabla^{2} V\right. \\
& \left.+\nabla^{2} \phi * \nabla^{3} \phi\right) d \tilde{V} \\
\leq & -\int_{B_{\tilde{g}}\left(x_{0}, r+1\right)}|\nabla \mathrm{Rm}|_{\tilde{g}} \xi^{2} w^{n-2} d \tilde{V}+C_{18} \int_{B_{\tilde{g}}\left(x_{0}, r+1\right)}|\nabla \mathrm{Rm}|_{\tilde{g}}|\operatorname{Rm}|_{\tilde{g}} \xi w^{n-2} d \tilde{V} \\
& +C_{18} \int_{B_{\tilde{g}}\left(x_{0}, r+1\right)}|\nabla \operatorname{Rm}|_{\tilde{g}}|\operatorname{Rm}|_{\tilde{g}} \xi^{2} w^{n-3}\left(|\operatorname{Rm}|_{\tilde{g}}|\nabla \operatorname{Rm}|_{\tilde{g}}+|\nabla V|_{\tilde{g}}\left|\nabla^{2} V\right|_{\tilde{g}}\right. \\
& \left.+\left|\nabla^{2} \phi\right|_{\tilde{g}}\left|\nabla^{3} \phi\right|_{\tilde{g}}\right) d \tilde{V}
\end{aligned}
$$


since $\nabla g=\tilde{\nabla} g+g^{-1} * \tilde{\nabla} g * g \lesssim 1$. By the inductive hypothesis, we have

$$
\begin{aligned}
& \int_{B_{\tilde{g}}\left(x_{0}, r+1\right)}|\nabla \operatorname{Rm}| \tilde{g}|\operatorname{Rm}|_{\tilde{g}} \xi w^{n-2} d \tilde{V} \\
\lesssim & \int_{B_{\tilde{g}}\left(x_{0}, r+1\right)} w^{n-2}|\nabla \operatorname{Rm}| \tilde{\tilde{g}} d \tilde{V}+\int_{B_{\tilde{g}}\left(x_{0}, r+1\right)} w^{n-2}|\operatorname{Rm}|_{\tilde{g}}^{2} d \tilde{V} \\
\lesssim & \int_{B_{\tilde{g}}\left(x_{0}, r+1\right)} u^{n-2} v d \tilde{V}+\int_{B_{\tilde{g}}\left(x_{0}, r+1\right)} u^{n-1} d \tilde{V} \\
\lesssim & 1+\int_{B_{\tilde{g}}\left(x_{0}, r+1\right)} u^{n-2} v d V,
\end{aligned}
$$

and

$$
\begin{aligned}
& \int_{B_{\tilde{g}}\left(x_{0}, r+1\right)} w^{n-3}|\nabla \operatorname{Rm}|_{\tilde{g}}|\operatorname{Rm}|_{\tilde{g}}\left(|\operatorname{Rm}|_{\tilde{g}}|\nabla \operatorname{Rm}|_{\tilde{g}}+|\nabla V|_{\tilde{g}}\left|\nabla^{2} V\right|_{\tilde{g}}\right. \\
& \left.+\left|\nabla^{2} \phi\right|_{\tilde{g}}\left|\nabla^{3} \phi\right|_{\tilde{g}}\right) d \tilde{V} \\
\lesssim & \int_{B_{\tilde{g}}\left(x_{0}, r+1\right)} w^{n-3} u v d \tilde{V}+\int_{B_{\tilde{g}}\left(x_{0}, r+1\right)} w^{n-3} u^{1 / 2} v^{1 / 2}\left(u^{1 / 2} v^{1 / 2}+u^{1 / 2} v^{1 / 2}\right) d \tilde{V} \\
\lesssim & \int_{B_{\tilde{g}}\left(x_{0}, r+1\right)} u^{n-2} v d V ;
\end{aligned}
$$

hence the first term on the right-hand side of 3.125$)$ is bounded from above by

$$
1+\int_{B_{\tilde{g}}\left(x_{0}, r+1\right)} u^{n-2} v d V
$$

up to a uniform positive multiple. Since $|\mathrm{Rm}|_{g}^{2} \lesssim 1+|\nabla \tilde{\nabla} g|_{\tilde{g}}^{2}$ by the equation (90) in page 277 of [26], it follows that the sum of the third and forth terms of the right-hand side of (3.125) is bounded from above by

$$
\begin{aligned}
& \int_{B_{\tilde{g}}\left(x_{0}, r+1\right)} w^{n-2} \xi^{2} * 2 \mathrm{Rm} *\left(g^{-1} * V * \nabla \mathrm{Rm}+g^{-1} * \mathrm{Rm} * \nabla V\right) d \tilde{V} \\
\leq & C_{19} \int_{B_{\tilde{g}\left(x_{0}, r+1\right)}} w^{n-2} \xi^{2}\left(|\nabla \mathrm{Rm}|_{\tilde{g}}|\mathrm{Rm}|_{\tilde{g}}+|\mathrm{Rm}|_{\tilde{g}}^{2}|\nabla V|_{\tilde{g}}\right) d \tilde{V} \\
\leq & \frac{1}{2} C_{19} \int_{B_{\tilde{g}}\left(x_{0}, r+1\right)} w^{n-2} \xi^{2}\left(|\nabla \mathrm{Rm}|_{\tilde{g}}^{2}+|\operatorname{Rm}|_{\tilde{g}}^{2}\right) d \tilde{V} \\
& +\frac{1}{2} C_{19} \int_{B_{\tilde{g}}\left(x_{0}, r+1\right)} w^{n-1} \xi^{2}|\mathrm{Rm}|_{\tilde{g}} d \tilde{V} \\
\leq & \frac{1}{2} C_{19} \int_{B_{\tilde{g}}\left(x_{0}, r+1\right)} w^{n-2} v d \tilde{V}+\frac{1}{2} C_{19} \int_{B_{\tilde{g}}\left(x_{0}, r+1\right)} w^{n-1} d \tilde{V} \\
& +C_{20} \int_{B_{\tilde{g}\left(x_{0}, r+1\right)} w^{n-1} \xi^{2}\left(1+|\nabla \tilde{\nabla} g|_{\tilde{g}}\right) \xi^{2} d \tilde{V}} \\
\leq & \frac{1}{8 C_{17}} \int_{B_{\tilde{g}}\left(x_{0}, r+1\right)} w^{n-1} \xi^{2}\left|\tilde{\nabla}^{2} g\right|_{\tilde{g}}^{2} d \tilde{V}+C_{21} \int_{B_{\tilde{g}}\left(x_{0}, r+1\right)} u^{n-2} v d V+C_{21},
\end{aligned}
$$

because of the inductive hypothesis and $V=g^{-1} * \tilde{\nabla} g \lesssim 1$. Similarly, the second term on the right-hand side of 3.125 is bounded from above by (up to a uniform 
positive multiple)

$$
\begin{aligned}
& \int_{B_{\tilde{g}}\left(x_{0}, r+1\right)} w^{n-2} \xi^{2} * g^{*-2} * \mathrm{Rm}^{* 3} d \tilde{V} \\
\lesssim & \int_{B_{\tilde{g}}\left(x_{0}, r+1\right)} w^{n-2} \xi^{2} * g^{*-2} * \mathrm{Rm}^{* 2} *(1+\nabla \tilde{\nabla} g) d \tilde{V} \\
\lesssim & \int_{B_{\tilde{g}}\left(x_{0}, r+1\right)} w^{n-1} d \tilde{V}+\int_{B_{\tilde{g}}\left(x_{0}, r+1\right)} w^{n-2} \xi^{2} * \mathrm{Rm}^{* 2} * g^{*-2} * \nabla \tilde{\nabla} g d \tilde{V} \\
\lesssim & 1+\int_{B_{\tilde{g}}\left(x_{0}, r+1\right)} \tilde{\nabla} g *\left(\nabla \xi * \xi * \mathrm{Rm}^{* 2}+\xi^{2} * \nabla \mathrm{Rm} * \mathrm{Rm}\right) * g^{*-2} * w^{n-2} d \tilde{V} \\
& +\int_{B_{\tilde{g}}\left(x_{0}, r+1\right)} \mathrm{Rm}^{* 2} * w^{n-3} *\left(\mathrm{Rm} * \nabla \mathrm{Rm}+\nabla V * \nabla^{2} V+\nabla^{2} \phi * \nabla^{3} \phi\right) d \tilde{V} \\
\lesssim & 1+\int_{B_{\tilde{g}}\left(x_{0}, r+1\right)}(u+v) u^{n-2} d V+\int_{B_{\tilde{g}}\left(x_{0}, r+1\right)} u u^{n-3}(u+v) d V \\
\lesssim & 1+\int_{B_{\tilde{g}}\left(x_{0}, r+1\right)} u^{n-2} v d V,
\end{aligned}
$$

by the equation (84) in page 276 of [26], $g$ is equivalent to $\tilde{g}$, and the inductive hypothesis. The last term on the right-hand side of (3.125) is bounded from above by (up to a uniform positive multiple)

$$
\begin{aligned}
& \int_{B_{\tilde{g}}\left(x_{0}, r+1\right)} w^{n-2} \xi^{2} * \mathrm{Rm} * \nabla^{2} \phi * \nabla^{2} \phi d \tilde{V} \\
\lesssim & \int_{B_{\tilde{g}}\left(x_{0}, r+1\right)} \nabla \phi * \nabla\left(w^{n-2} \xi^{2} * \mathrm{Rm} * \nabla^{2} \phi\right) d \tilde{V} \\
= & \int_{B_{\tilde{g}}\left(x_{0}, r+1\right)} \nabla \phi *\left(\xi * \nabla \xi * \mathrm{Rm} * \nabla^{2} \phi+\xi^{2} * \nabla \mathrm{Rm} * \nabla^{2} \phi\right. \\
& \left.+\xi^{2} * \mathrm{Rm} * \nabla^{3} \phi\right) w^{n-2} d \tilde{V}+\int_{B_{\tilde{g}}\left(x_{0}, r+1\right)} \nabla \phi * \xi^{2} * \mathrm{Rm} * \nabla^{2} \phi * w^{n-3} \\
& *\left(\mathrm{Rm} * \nabla \mathrm{Rm}+\nabla V * \nabla^{2} V+\nabla^{2} \phi * \nabla^{3} \phi\right) d \tilde{V} \\
\lesssim & \int_{B_{\tilde{g}}\left(x_{0}, r+1\right)} w^{n-2}\left(w+v^{1 / 2} w^{1 / 2}\right) d \tilde{V}+\int_{B_{\tilde{g}}\left(x_{0}, r+1\right)} w w^{n-3}(w+v) d \tilde{V} \\
\lesssim & \int_{B_{\tilde{g}}\left(x_{0}, r+1\right)} u^{n-1} d V+\int_{B_{\tilde{g}}\left(x_{0}, r+1\right)} u^{n-2} v d V \lesssim 1+\int_{B_{\tilde{g}}\left(x_{0}, r+1\right)} u^{n-2} v d V .
\end{aligned}
$$

Note that when we do the integration by parts, we may replace $\tilde{g}$ by $g$ since $g$ is equivalent $\tilde{g}$, so that we have no extra terms $\nabla \tilde{g}$ and $\nabla \tilde{g}^{-1}$. Therefore, substituting those estimates into 3.125 implies

$$
C_{17} J_{1} \leq \frac{1}{8} \int_{B_{\tilde{g}}\left(x_{0}, r+1\right)} w^{n-1} \xi^{2}\left|\tilde{\nabla}^{2} g\right|_{\tilde{g}}^{2} d \tilde{V}+C_{22} \int_{B_{\tilde{g}}\left(x_{0}, r+1\right)} u^{n-2} v d V+C_{22}
$$


By (3.125), we have

$$
\begin{aligned}
J_{2}= & \int_{B_{\tilde{g}}\left(x_{0}, r+1\right)} w^{n-2} \xi^{2} 2\left\langle\nabla V, \Delta \nabla V+g^{*-3} * \nabla \tilde{\nabla} g * \mathrm{Rm}+g^{*-3} * \tilde{\nabla} g * \nabla \mathrm{Rm}\right. \\
(3.127) \quad & g^{*-2} * \nabla \tilde{\nabla} g * \nabla V+g^{*-2} * \tilde{\nabla} g * \nabla^{2} V \\
& \left.+g^{*-2} * \nabla \tilde{\nabla} g *(\nabla \phi)^{* 2}+g^{*-2} * \tilde{\nabla} g * \nabla \phi * \nabla^{2} \phi\right\rangle_{\tilde{g}} d \tilde{V} .
\end{aligned}
$$

As before, the first term on the right-hand side of (3.127) is bounded from above by (up to a uniform positive multiple)

$$
\begin{aligned}
& 2 \int_{B_{\tilde{g}}\left(x_{0}, r+1\right)} w^{n-2} \xi^{2}\langle\nabla V, \Delta \nabla V\rangle_{\tilde{g}} d \tilde{V} \\
= & -2 \int_{B_{\tilde{g}}\left(x_{0}, r+1\right)}\left\langle\nabla_{\beta} \nabla V, \nabla_{\alpha}\left(g^{\alpha \beta} \xi^{2} w^{n-2} \nabla V\right)\right\rangle_{\tilde{g}} d \tilde{V} \\
\leq & -\int_{B_{\tilde{g}}\left(x_{0}, r+1\right)}\left|\nabla^{2} V\right|_{\tilde{g}}^{2} \xi^{2} w^{n-2} d \tilde{V} \\
& +\int_{B_{\tilde{g}}\left(x_{0}, r+1\right)} \nabla^{2} V * \nabla V * g^{-1}\left[\xi * \nabla \xi * w^{n-2}+\xi * w^{n-3} *(\mathrm{Rm} * \nabla \mathrm{Rm}\right. \\
& \left.\left.+\nabla V * \nabla^{2} V+\nabla^{2} \phi * \nabla^{3} \phi\right)\right] d \tilde{V} \\
\lesssim & \int_{B_{\tilde{g}}\left(x_{0}, r+1\right)} w^{n-2} v^{1 / 2} w^{1 / 2} d \tilde{V}+\int_{B_{\tilde{g}}\left(x_{0}, r+1\right)} v^{1 / 2} w^{1 / 2} w^{n-3} v^{1 / 2} w^{1 / 2} d \tilde{V} \\
\lesssim & \int_{B_{\tilde{g}}\left(x_{0}, r+1\right)} u^{n-1} d V+\int_{B_{\tilde{g}}\left(x_{0}, r+1\right)} u^{n-2} v d V \lesssim 1+\int_{B_{\tilde{g}}\left(x_{0}, r+1\right)} u^{n-2} v d V
\end{aligned}
$$

by the inductive hypothesis. By the Cauchy-Schwarz inequality, the sum of the second, third, forth, and fifth terms on the right-hand side of (3.127) is bounded from above by

$$
\begin{aligned}
& C_{23} \int_{B_{\tilde{g}\left(x_{0}, r+1\right)}} w^{n-2}|\nabla V|_{\tilde{g}}\left[w^{1 / 2}\left|\tilde{\nabla}^{2} g\right|_{\tilde{g}}+|\nabla \mathrm{Rm}|_{\tilde{g}}+\left|\nabla^{2} V\right|_{\tilde{g}}\right] \xi^{2} d \tilde{V} \\
\leq & \frac{1}{8 C_{17}} \int_{B_{\tilde{g}\left(x_{0}, r+1\right)}} w^{n-1} \xi^{2}\left|\tilde{\nabla}^{2} g\right|_{\tilde{g}}^{2} d \tilde{V}+C_{24} \int_{B_{\tilde{g}}\left(x_{0}, r+1\right)} w^{n-2} v d \tilde{V}+C_{24} \\
\leq & \frac{1}{8 C_{17}} \int_{B_{\tilde{g}}\left(x_{0}, r+1\right)} w^{n-1} \xi^{2}\left|\tilde{\nabla}^{2} g\right|_{\tilde{g}}^{2} d \tilde{V}+C_{25} \int_{B_{\tilde{g}}\left(x_{0}, r+1\right)} u^{n-2} v d V+C_{25} .
\end{aligned}
$$

The rest terms on the right-hand side of (3.127) is bounded from above by (up to a uniform positive multiple)

$$
\begin{aligned}
& \int_{B_{\tilde{g}}\left(x_{0}, r+1\right)} w^{n-2} \xi^{2} * \nabla V * g^{*-2} *\left(\nabla \tilde{\nabla} g *(\nabla \phi)^{* 2}+\tilde{\nabla} g * \nabla \phi * \nabla^{2} \phi\right) d \tilde{V} \\
\lesssim & \int_{B_{\tilde{g}}\left(x_{0}, r+1\right)} w^{n-2} w^{1 / 2}|\nabla \tilde{\nabla} g|_{\tilde{g}} d \tilde{V}+\int_{B_{\tilde{g}}\left(x_{0}, r+1\right)} w^{n-2} w^{1 / 2} w^{1 / 2} d \tilde{V} \\
\lesssim & \int_{B_{\tilde{g}}\left(x_{0}, r+1\right)} u^{n-1} d V+\int_{B_{\tilde{g}}\left(x_{0}, r+1\right)} u^{n-2}|\nabla \tilde{\nabla} g|_{\tilde{g}}^{2} d \tilde{V} \lesssim 1
\end{aligned}
$$


by the inductive hypothesis. Hence

$$
C_{17} J_{2} \leq \frac{1}{8} \int_{B_{\tilde{g}}\left(x_{0}, r+1\right)} w^{n-1} \xi^{2}\left|\tilde{\nabla}^{2} g\right|_{\tilde{g}}^{2} d \tilde{V}+C_{26} \int_{B_{\tilde{g}}\left(x_{0}, r+1\right)} u^{n-2} v d V+C_{26}
$$

According to 3.75 ,

$$
\begin{aligned}
J_{3}= & \int_{B_{\tilde{g}}\left(x_{0}, r+1\right)} w^{n-2} \xi^{2} 2\left\langle\nabla^{2} \phi, \Delta \nabla^{2} \phi+g^{*-2} * \mathrm{Rm} * \nabla^{2} \phi+\beta_{2} \nabla^{2} \phi\right. \\
& +g^{-1} *(\nabla \phi)^{* 2} * \nabla^{2} \phi+g^{-1} * \nabla \phi * \nabla^{2} \phi+g^{-1} *\left(\nabla^{2} \phi\right)^{* 2} \\
& \left.+g^{*-2} * \mathrm{Rm} *(\nabla \phi)^{* 2}+g^{-1} * V * \nabla^{3} \phi+g^{-1} * \nabla V * \nabla^{2} \phi\right\rangle_{\tilde{g}} d \tilde{V}
\end{aligned}
$$

By the integration by parts, the first term on the right-hand side of 3.129 equals

$$
\begin{aligned}
& -2 \int_{B_{\tilde{g}}\left(x_{0}, r+1\right)}\left\langle\nabla_{\beta} \nabla^{2} \phi, \nabla_{\alpha}\left(\xi^{2} w^{n-2} g^{\alpha \beta} \nabla^{2} \phi\right)\right\rangle_{\tilde{g}} d \tilde{V} \\
= & -2 \int_{B_{\tilde{g}}\left(x_{0}, r+1\right)}\left\langle\nabla_{\beta} \nabla^{2} \phi, \xi^{2} g^{\alpha \beta} w^{n-2} \nabla_{\alpha} \nabla^{2} \phi\right\rangle_{\tilde{g}} d \tilde{V}+\int_{B_{\tilde{g}}\left(x_{0}, r+1\right)} \xi^{2} \nabla^{3} \phi * \nabla^{2} \phi \\
& * g^{-1} * w^{n-3} *\left(\mathrm{Rm} * \nabla \mathrm{Rm}+\nabla V * \nabla^{2} V+\nabla^{2} \phi * \nabla^{3} \phi\right) d \tilde{V} \\
& +\int_{B_{\tilde{g}}\left(x_{0}, r+1\right)} \nabla^{3} \phi * \nabla^{2} \phi * g^{-1} * w^{n-2} * \nabla \xi * \xi d \tilde{V} \\
\lesssim & -\int_{B_{\tilde{g}}\left(x_{0}, r+1\right)} \xi^{2} w^{n-2}\left|\nabla^{3} \phi\right|_{\tilde{g}}^{2} d \tilde{V}+\int_{B_{\tilde{g}}\left(x_{0}, r+1\right)} v^{1 / 2} w^{1 / 2} w^{n-3} w^{1 / 2} v^{1 / 2} d \tilde{V} \\
& +\int_{B_{\tilde{g}}\left(x_{0}, r+1\right)} v^{1 / 2} w^{1 / 2} w^{n-2} d \tilde{V} \lesssim 1+\int_{B_{\tilde{g}}\left(x_{0}, r+1\right)} w^{n-2} v d \tilde{V} .
\end{aligned}
$$

The rest terms on the right-hand side of (3.129) are bounded from above by (up to a uniform positive multiple)

$$
\begin{aligned}
\int_{B_{\tilde{g}}\left(x_{0}, r+1\right)} w^{n-2}\left(w+w^{1 / 2}+v^{1 / 2}\right) d \tilde{V} & \lesssim \int_{B_{\tilde{g}}\left(x_{0}, r+1\right)} w^{n-2}(1+w+v) d \tilde{V} \\
& \lesssim 1+\int_{B_{\tilde{g}}\left(x_{0}, r+1\right)} u^{n-2} v d V
\end{aligned}
$$

by the inductive hypothesis. Hence

$$
J_{3} \lesssim 1+\int_{B_{\tilde{g}}\left(x_{0}, r+1\right)} u^{n-2} v d V
$$

Combining 3.126), 3.128, and 3.130, we find that

$$
J \leq \frac{1}{4} \int_{B_{\tilde{g}}\left(x_{0}, r+1\right)} w^{n-1} \xi^{2}\left|\tilde{\nabla}^{2} g\right|_{\tilde{g}}^{2} d \tilde{V}+C_{27} \int_{B_{\tilde{g}}\left(x_{0}, r+1\right)} u^{n-2} v d V+C_{27} .
$$

Substituting (3.124) and (3.131) into the definition of $K$ yields

$$
\begin{aligned}
\frac{d}{d t} \int_{B_{\tilde{g}}\left(x_{0}, r+1\right)} w^{n-1}|\tilde{\nabla} g|_{\tilde{g}}^{2} \xi^{2} d \tilde{V} \leq & -\frac{1}{4} \int_{B_{\tilde{g}}\left(x_{0}, r+1\right)} w^{n-1} \xi^{2}\left|\tilde{\nabla}^{2} g\right|_{\tilde{g}}^{2} d \tilde{V}+C_{28} \\
& +C_{28} \int_{B_{\tilde{g}}\left(x_{0}, r+1\right)} u^{n-2} v d V \\
& +C_{28} \int_{B_{\tilde{g}}\left(x_{0}, r+1\right)} w^{n-1}\left|\tilde{\nabla}^{2} \phi\right|_{\tilde{g}} \xi d \tilde{V} .
\end{aligned}
$$


Define

$$
\begin{aligned}
L:= & \frac{d}{d t} \int_{B_{\tilde{g}}\left(x_{0}, r+1\right)} w^{n-1}|\tilde{\nabla} \phi|_{\tilde{g}}^{2} \xi^{2} d \tilde{V}=2 \int_{B_{\tilde{g}}\left(x_{0}, r+1\right)} w^{n-1} \xi^{2}\left\langle\tilde{\nabla} \phi, \partial_{t} \tilde{\nabla} \phi\right\rangle_{\tilde{g}} d \tilde{V} \\
= & 2 \int_{B_{\tilde{g}}\left(x_{0}, r+1\right)} w^{n-1} \xi^{2}\left\langle\tilde{\nabla} \phi, g^{\alpha \beta} \tilde{\nabla}_{\alpha} \tilde{\nabla}{ }_{\beta} \tilde{\nabla} \phi\right\rangle_{\tilde{g}} d \tilde{V} \\
& +\int_{B_{\tilde{g}}\left(x_{0}, r+1\right)} w^{n-1} \xi^{2} \tilde{\nabla} \phi *\left(g^{*-2} * \widetilde{\operatorname{Rm}} * \tilde{\nabla} \phi+g^{*-2} * \tilde{\nabla} g * \tilde{\nabla}^{2} \phi\right. \\
& \left.+g^{*-2} * \tilde{\nabla} g *(\tilde{\nabla} \phi)^{* 2}+g^{-1} * \tilde{\nabla} \phi * \tilde{\nabla}^{2} \phi+\tilde{\nabla} \phi\right) d \tilde{V}:=L_{1}+L_{2} .
\end{aligned}
$$

For $L_{2}$, we have

$$
L_{2} \lesssim \int_{B_{\tilde{g}}\left(x_{0}, r+1\right)} w^{n-1} \xi\left(1+\left|\tilde{\nabla}^{2} \phi\right| \tilde{g}\right) d \tilde{V} \lesssim 1+\int_{B_{\tilde{g}}\left(x_{0}, r+1\right)} w^{n-1} \xi\left|\tilde{\nabla}^{2} \phi\right|_{\tilde{g}} d \tilde{V}
$$

by the inductive hypothesis. Taking the integration by parts on $L_{1}$ implies

$$
\begin{aligned}
L_{1}= & -2 \int_{B_{\tilde{g}}\left(x_{0}, r+1\right)}\left\langle\tilde{\nabla}_{\beta} \tilde{\nabla} \phi, \tilde{\nabla}_{\alpha}\left(g^{\alpha \beta} w^{n-1} \xi^{2} \tilde{\nabla} \phi\right)\right\rangle_{\tilde{g}} d \tilde{V} \\
= & -2 \int_{B_{\tilde{g}}\left(x_{0}, r+1\right)}\left\langle\tilde{\nabla}_{\beta} \tilde{\nabla} \phi, g^{\alpha \beta} w^{n-1} \xi^{2} \tilde{\nabla} \alpha \tilde{\nabla} \phi\right\rangle_{\tilde{g}} d \tilde{V} \\
& +\int_{B_{\tilde{g}}\left(x_{0}, r+1\right)} \tilde{\nabla}^{2} \phi * \tilde{\nabla} \phi *\left(g^{*-2} * \tilde{\nabla} g * w^{n-1} * \xi^{2}+g^{-1} * \xi * \tilde{\nabla} \xi * w^{n-1}\right. \\
& \left.+g^{-1} * \xi^{2} * w^{n-2} *\left(\mathrm{Rm} * \tilde{\nabla} \mathrm{Rm}+\nabla V * \tilde{\nabla} \nabla V+\nabla^{2} \phi * \tilde{\nabla} \nabla^{2} \phi\right)\right) d \tilde{V} \\
\leq & -\int_{B_{\tilde{g}}\left(x_{0}, r+1\right)} w^{n-1} \xi^{2}\left|\tilde{\nabla}^{2} \phi\right|_{\tilde{g}}^{2} d \tilde{V}+C_{29} \int_{B_{\tilde{g}}\left(x_{0}, r+1\right)} w^{n-1} \xi\left|\tilde{\nabla}^{2} \phi\right|_{\tilde{g}} d \tilde{V} \\
& +C_{29} \int_{B_{\tilde{g}}\left(x_{0}, r+1\right)} w^{n-2} \xi^{2}\left|\tilde{\nabla}^{2} \phi\right|_{\tilde{g}} w^{1 / 2}\left(w^{1 / 2}+v^{1 / 2}\right) d \tilde{V} \\
= & -\int_{B_{\tilde{g}}\left(x_{0}, r+1\right)} w^{n-1} \xi^{2}\left|\tilde{\nabla}^{2} \phi\right|_{\tilde{g}}^{2} d \tilde{V}+2 C_{29} \int_{B_{\tilde{g}}\left(x_{0}, r+1\right)} \xi w^{n-1}\left|\tilde{\nabla}^{2} \phi\right|_{\tilde{g}} d \tilde{V} \\
& +C_{29} \int_{B_{\tilde{g}}\left(x_{0}, r+1\right)}\left(\xi w^{\frac{n-1}{2}}\left|\tilde{\nabla}^{2} \phi\right|_{\tilde{g}}\right)\left(\xi w^{\frac{n-2}{2}} v^{1 / 2}\right) d \tilde{V} \\
\leq & -\frac{3}{4} \int_{B_{\tilde{g}}\left(x_{0}, r+1\right)} w^{n-1} \xi^{2}\left|\tilde{\nabla}^{2} \phi\right|_{\tilde{g}}^{2} d \tilde{V}+C_{30} \int_{B_{\tilde{g}}\left(x_{0}, r+1\right)} w^{n-1} \xi\left|\tilde{\nabla}^{2} \phi\right|_{\tilde{g}} d \tilde{V} \\
& +C_{30} \int_{B_{\tilde{g}}\left(x_{0}, r+1\right)} u^{n-2} v d V
\end{aligned}
$$

since

$$
\begin{aligned}
\tilde{\nabla} \mathrm{Rm} & =\nabla \mathrm{Rm}+g^{-1} * \tilde{\nabla} g * \mathrm{Rm} \lesssim v^{1 / 2}+w^{1 / 2} \\
\tilde{\nabla} \nabla V & =\nabla^{2} V+g^{-1} * \tilde{\nabla} g * \nabla V \lesssim v^{1 / 2}+w^{1 / 2} \\
\tilde{\nabla} \nabla^{2} \phi & =\nabla^{3} \phi+g^{-1} * \tilde{\nabla} g * \nabla^{2} \phi \lesssim v^{1 / 2}+w^{1 / 2}
\end{aligned}
$$

Therefore

$$
L \leq-\frac{1}{2} \int_{B_{\tilde{g}}\left(x_{0}, r+1\right)} w^{n-1} \xi^{2}\left|\tilde{\nabla}^{2} \phi\right|_{\tilde{g}}^{2} d \tilde{V}+C_{31} \int_{B_{\tilde{g}}\left(x_{0}, r+1\right)} u^{n-2} v d V+C_{31}
$$


From 3.132 and 3.133 , we arrive at

$$
\begin{aligned}
& \frac{d}{d t}\left(\int_{B_{\tilde{g}}\left(x_{0}, r+1\right)} w^{n-1} \xi^{2}\left(|\tilde{\nabla} g|_{\tilde{g}}^{2}+|\tilde{\nabla} \phi|_{\tilde{g}}^{2}\right) d \tilde{V}\right) \\
\leq & -\frac{1}{4} \int_{B_{\tilde{g}}\left(x_{0}, r+1\right)} w^{n-1} \xi^{2}\left(\left|\tilde{\nabla}^{2} g\right|_{\tilde{g}}^{2}+\left|\tilde{\nabla}^{2} \phi\right|_{\tilde{g}}^{2}\right) d \tilde{V} \\
& +C_{32} \int_{B_{\tilde{g}}\left(x_{0}, r+1\right)} u^{n-2} v d V+C_{32} .
\end{aligned}
$$

Consequently,

$$
\int_{0}^{T} \int_{B_{\tilde{g}}\left(x_{0}, r+1\right)} w^{n-1} \xi^{2}|\tilde{\nabla} g|_{\tilde{g}}^{2} d \tilde{V} d t \lesssim 1
$$

in particular,

$$
\int_{0}^{T} \int_{B_{\tilde{g}}\left(x_{0}, r+1\right)} u^{n-1}|\nabla \tilde{\nabla} g|_{g}^{2} \xi^{2} d V d t \lesssim 1 .
$$

By the equations (90) in page 277 and (108) in page 281 of 26, together with $\nabla^{2} \phi=\tilde{\nabla}^{2}+g^{-1} * \tilde{\nabla} g * \tilde{\nabla} \phi$, we have

$$
|\mathrm{Rm}|_{g}^{2}+|\nabla V|_{g}^{2}+\left|\nabla^{2} \phi\right|_{g}^{2} \lesssim 1+|\nabla \tilde{\nabla} g|_{g}^{2}
$$

using the estimate (3.134), we obtain

$$
\int_{0}^{T} \int_{B_{\tilde{g}}\left(x_{0}, r+1\right)} u^{n} d V d t \lesssim 1 .
$$

As in the proof of Lemma 3.12, we can show that

$$
\int_{B_{\tilde{g}}\left(x_{0}, r+1\right)} u^{n} \xi^{2} d V+\frac{1}{8} \int_{0}^{t} \int_{B_{\tilde{g}}\left(x_{0}, r+1\right)} u^{n-1} v \xi^{2} d V d t \lesssim 1
$$

by the previous estimates and inductive hypothesis. Thus the lemma is also true for $s=n$.

We now can prove the following theorem, as in [26] where use the equations (3.118, 3.119, 3.120, and Lemma 3.13.

Theorem 3.14. We have

$$
\sup _{M \times[0, T]}|\operatorname{Rm}|_{g}^{2} \lesssim 1, \quad \sup _{M \times[0, T]}|\nabla V|_{g}^{2} \lesssim 1, \quad \sup _{M \times[0, T]}\left|\nabla^{2} \phi\right|_{g}^{2} \lesssim 1,
$$

where $\lesssim$ depend on $n, k_{0}, k_{1}, k_{2}, \alpha_{1}, \beta_{1}, \beta_{2}$.

By the same argument used in [19, 26], we have

Theorem 3.15. Let $(M, \tilde{g})$ be a complete noncompact Riemannian m-manifold with bounded Riemann curvature $\left|\operatorname{Rm}_{\tilde{g}}\right|_{\tilde{g}}^{2} \leq k_{0}$, and $\tilde{\phi}$ a smooth function on $M$ satisfying

$$
|\tilde{\phi}|^{2}+\left|\nabla_{\tilde{g}} \tilde{\phi}\right|_{\tilde{g}}^{2} \leq k_{1}, \quad\left|\nabla_{\tilde{g}}^{2} \tilde{\phi}\right|_{\tilde{g}}^{2} \leq k_{2} .
$$

Then there exists a positive constant $T=T\left(m, k_{0}, k_{1}, \alpha_{1}, \beta_{1}, \beta_{2}\right)>0$ such that the regular- $\left(\alpha_{1}, 0, \beta_{1}, \beta_{2}\right)$-flow

$$
\begin{aligned}
\partial_{t} \hat{g}(t) & =-2 \operatorname{Ric}_{\hat{g}(t)}+2 \alpha_{1} \nabla_{\hat{g}(t)} \hat{\phi}(t) \otimes \nabla_{\hat{g}(t)} \hat{\phi}(t), \\
\partial_{t} \hat{\phi}(t) & =\Delta_{\hat{g}(t)} \hat{\phi}(t)+\beta_{1}\left|\nabla_{\hat{g}(t)} \hat{\phi}(t)\right|_{\hat{g}(t)}^{2}+\beta_{2} \hat{\phi}(t), \\
(\hat{g}(0), \hat{\phi}(0)) & =(\tilde{g}, \tilde{\phi})
\end{aligned}
$$


has a smooth solution $(\hat{g}(t), \hat{\phi}(t))$ on $M \times[0, T]$ and satisfies the following estimate

$$
\frac{1}{C_{1}} \tilde{g} \leq \hat{g}(t) \leq C_{1} \tilde{g}, \quad\left|\operatorname{Rm}_{\hat{g}(t)}\right|_{\hat{g}(t)}^{2}+|\hat{\phi}(t)|^{2}+\left|\nabla_{\hat{g}(t)} \hat{\phi}(t)\right|_{\hat{g}(t)}^{2}+\left|\nabla_{\hat{g}(t)}^{2} \hat{\phi}(t)\right|_{\hat{g}(t)}^{2} \leq C_{2}
$$

on $M \times[0, T]$, where $C_{1}, C_{2}$ are uniform positive constants depending only on $m, k_{0}, k_{1}, k_{2}, \alpha_{1}, \beta_{1}, \beta_{2}$. flow

Suppose that $(\hat{g}(t), \hat{\phi}(t))$ is a smooth solution to the regular- $\left(\alpha_{1}, 0, \beta_{1}-\alpha_{2}, \beta_{2}\right)$ -

$$
\begin{aligned}
\partial_{t} \hat{g}(t) & =-2 \operatorname{Ric}_{\hat{g}(t)}+2 \alpha_{1} \nabla_{\hat{g}(t)} \hat{\phi}(t) \otimes \nabla_{\hat{g}(t)} \hat{\phi}(t), \\
\partial_{t} \hat{\phi}(t) & =\Delta_{\hat{g}(t)} \hat{\phi}(t)+\left(\beta_{1}-\alpha_{2}\right)\left|\nabla_{\hat{g}(t)} \hat{\phi}(t)\right|_{\hat{g}(t)}^{2}+\beta_{2} \hat{\phi}(t), \\
(\hat{g}(0), \hat{\phi}(0)) & =(\tilde{g}, \tilde{\phi}) .
\end{aligned}
$$

Consider a 1-parameter family of diffeomorphisms $\Phi(t): M \rightarrow M$ by

$$
\frac{d}{d t} \Phi(t)=\alpha_{2} \nabla_{\hat{g}(t)} \hat{\phi}(t), \quad \Phi(0)=\operatorname{Id}_{M} .
$$

If we define

$$
g(t):=[\Phi(t)]^{*} \hat{g}(t), \quad \phi(t):=[\Phi(t)]^{*} \hat{\phi}(t),
$$

then

$$
\begin{aligned}
\partial_{t} g(t) & =[\Phi(t)]^{*}\left(\partial_{t} \hat{g}(t)\right)+\alpha_{1}[\Phi(t)]^{*}\left(\mathscr{L}_{\nabla_{\hat{g}(t)} \hat{\phi}(t)} \hat{g}(t)\right) \\
& =[\Phi(t)]^{*}\left(-2 \operatorname{Rm}_{\hat{g}(t)}+2 \alpha_{1} \nabla_{\hat{g}(t)} \hat{\phi}(t) \otimes \nabla_{\hat{g}(t)} \hat{\phi}(t)\right)+2 \alpha_{2}[\Phi(t)]^{*} \nabla_{\hat{g}(t)}^{2} \hat{\phi}(t) \\
& =-2 \operatorname{Rm}_{g(t)}+2 \alpha_{1} \nabla_{g(t)} \phi(t) \otimes \nabla_{g(t)} \phi(t)+2 \alpha_{2} \nabla_{g(t)}^{2} \phi(t), \\
\partial_{t} \phi(t) & =\partial_{t}(\hat{\phi}(t) \cdot \Phi(t)) \\
& =\partial_{t} \hat{\phi}(t) \circ \Phi(t)+\alpha_{2}\left|\nabla_{\hat{g}(t)} \hat{\phi}(t)\right|_{\hat{g}(t)}^{2} \\
& =\Delta_{g(t)} \phi(t)+\beta_{1}\left|\nabla_{g(t)} \phi(t)\right|_{g(t)}^{2}+\beta_{2} \phi(t) .
\end{aligned}
$$

If we furthermore have $|\hat{\phi}(t)|^{2} \lesssim 1$ and $\left|\nabla_{\hat{g}(t)} \hat{\phi}(t)\right|_{\hat{\phi}}^{2} \lesssim 1$ on $M \times[0, T]$, using the standard theory of ordinary differential equations we have that the system (3.136) has a unique smooth solution $\Phi(t)$ on $M \times[0, T]$. Therefore $(g(t), \phi(t))$ defined in 3.137) are also smooth on $M \times[0, T]$ and satisfies the above system of equations.

Theorem 3.16. Let $(M, \tilde{g})$ be a complete noncompact Riemannian m-manifold with bounded Riemann curvature $\left|\operatorname{Rm}_{\tilde{g}}\right|_{\tilde{g}}^{2} \leq k_{0}$, and $\tilde{\phi}$ a smooth function on $M$ satisfying

$$
|\tilde{\phi}|^{2}+\left|\nabla_{\tilde{g}} \tilde{\phi}\right|_{\tilde{g}}^{2} \leq k_{1}, \quad\left|\nabla_{\tilde{g}}^{2} \tilde{\phi}\right|_{\tilde{g}}^{2} \leq k_{2} .
$$

Then there exists a positive constant $T=T\left(m, k_{0}, k_{1}, \alpha_{1}, \alpha_{2}, \beta_{1}, \beta_{2}\right)>0$ such that the regular- $\left(\alpha_{1}, \alpha_{2}, \beta_{1}, \beta_{2}\right)$-flow

$$
\begin{aligned}
\partial_{t} g(t) & =-2 \operatorname{Ric}_{g(t)}+2 \alpha_{1} \nabla_{g(t)} \phi(t) \otimes \nabla_{g(t)} \phi(t), \\
\partial_{t} \phi(t) & =\Delta_{g(t)} \phi(t)+\beta_{1}\left|\nabla_{g(t)} \phi(t)\right|_{g(t)}^{2}+\beta_{2} \phi(t), \\
(g(0), \phi(0)) & =(\tilde{g}, \phi)
\end{aligned}
$$


has a smooth solution $(g(t), \phi(t))$ on $M \times[0, T]$ and satisfies the following estimate

$$
\frac{1}{C_{1}} \tilde{g} \leq g(t) \leq C_{1} \tilde{g}, \quad\left|\operatorname{Rm}_{g(t)}\right|_{g(t)}^{2}+|\phi(t)|^{2}+\left|\nabla_{g(t)} \phi(t)\right|_{g(t)}^{2}+\left|\nabla_{g(t)}^{2} \phi(t)\right|_{g(t)}^{2} \leq C_{2}
$$

on $M \times[0, T]$, where $C_{1}, C_{2}$ are uniform positive constants depending only on $m, k_{0}, k_{1}, k_{2}, \alpha_{1}, \alpha_{2}, \beta_{1}, \beta_{2}$.

3.5. Higher order derivatives estimates. To complete the proof of Theorem 3.1. we need only to prove the higher order derivatives estimates (3.1). Suppose we have a smooth solution $(g(t), \phi(t))$ on $M \times[0, T]$ and satisfies

$$
\begin{aligned}
\partial_{t} g(t) & =-2 \operatorname{Ric}_{g(t)}+2 \alpha_{2} \nabla_{g(t)} \phi(t) \otimes \nabla_{g(t)} \phi(t)+2 \alpha_{2} \nabla_{g(t)}^{2} \phi(t), \\
\partial_{t} \phi(t) & =\Delta_{g(t)} \phi(t)+\beta_{1}\left|\nabla_{g(t)} \phi(t)\right|_{g(t)}^{2}+\beta_{2} \phi(t), \\
(g(0), \phi(0)) & =(\tilde{g}, \tilde{\phi})
\end{aligned}
$$

where $(M, \tilde{g})$ is a complete noncompact Riemannian $m$-manifold with bounded curvature $\left|\operatorname{Rm}_{\tilde{g}}\right|_{\tilde{g}}^{2} \leq k_{0}$ and $\tilde{\phi}$ is a smooth function on $M$ satisfying $|\tilde{\phi}|^{2}+\left|\nabla_{\tilde{g}} \tilde{\phi}\right|_{\tilde{g}}^{2} \leq k_{1}$ and $\left|\nabla_{\tilde{g}}^{2} \tilde{\phi}\right|_{\tilde{g}}^{2} \leq k_{2}$, and

$$
g(t) \approx \tilde{g}, \quad\left|\mathrm{Rm}_{g(t)}\right|_{g(t)}^{2}+|\phi(t)|^{2}+\left|\nabla_{g(t)} \phi(t)\right|_{g(t)}^{2}+\left|\nabla_{g(t)}^{2} \phi(t)\right|_{g(t)}^{2} \lesssim 1
$$

on $M \times[0, T]$, where $\lesssim$ or $\approx$ depends only on $m, k_{0}, k_{1}, k_{2}, \alpha_{1}, \alpha_{2}, \beta_{1}, \beta_{2}$.

Lemma 3.17. For any nonnegative integer $n$, there exist uniform positive constants $C_{k}$ depending only on $m, n, k_{0}, k_{1}, k_{2}, \alpha_{1}, \alpha_{2}, \beta_{1}, \beta_{2}$ such that

$$
\left|\nabla_{g(t)}^{n} \operatorname{Rm}_{g(t)}\right|_{g(t)}^{2}+\left|\nabla_{g(t)}^{n+2} \phi(t)\right|_{g(t)}^{2} \leq \frac{C_{n}}{t^{n}}
$$

on $M \times[0, T]$.

Proof. As before, we always write $\mathrm{Rm}:=\mathrm{Rm}_{g(t)}, \phi:=\phi(t)$, etc. From Lemma 2.5 , we have

(3.141) $\partial_{t} \mathrm{Rm}=\Delta \mathrm{Rm}+g^{*-2} * \mathrm{Rm}^{* 2}+\left(\nabla^{2} \phi\right)^{* 2}+g^{-1} * \nabla \mathrm{Rm} * \nabla \phi+g^{-1} * \mathrm{Rm} * \nabla^{2} \phi$.

Then the norm $|\mathrm{Rm}|^{2}$ of Riemann curvature evolves by

$$
\partial_{t}|\mathrm{Rm}|^{2}=2\left\langle\mathrm{Rm}, \partial_{t} \mathrm{Rm}\right\rangle_{g}+g^{*-3} * \partial_{t} g^{-1} * \mathrm{Rm}^{* 2}
$$

substituting (3.138) and 3.141) into above yields

$$
\begin{aligned}
\partial_{t}|\mathrm{Rm}|^{2}= & \Delta|\mathrm{Rm}|^{2}-2|\nabla \mathrm{Rm}|^{2}+g^{*-6} * \mathrm{Rm}^{* 3} \\
& +g^{*-4} * \mathrm{Rm} *\left(\nabla^{2} \phi\right)^{* 2}+g^{*-5} * \mathrm{Rm}^{* 2} *(\nabla \phi)^{* 2} \\
& +g^{*-5} * \mathrm{Rm}^{* 2} * \nabla^{2} \phi+g^{*-5} * \mathrm{Rm} * \nabla \mathrm{Rm} * \nabla \phi .
\end{aligned}
$$

Introduce a family of vector-valued tensor fields

$$
\boldsymbol{\Lambda}:=(\mathrm{Rm}, \nabla \phi),
$$

and define

$$
\left|\nabla^{k} \boldsymbol{\Lambda}\right|^{2}:=\left|\nabla^{k} \mathrm{Rm}\right|^{2}+\left|\nabla^{k+1} \phi\right|^{2}
$$

for each nonnegative integer $k$. According to 3.139 , 3.142 and the CauchySchwarz inequality, we have

$$
\partial_{t}|\mathrm{Rm}|^{2} \leq \Delta|\mathrm{Rm}|^{2}-\frac{3}{2}|\nabla \mathrm{Rm}|^{2}+C_{1} .
$$


Since $\partial_{t} \nabla \mathrm{Rm}=\nabla \partial_{t} \mathrm{Rm}+\partial_{t} \Gamma * \mathrm{Rm}$ and

$$
\begin{aligned}
\partial_{t} \Gamma & =g^{-1} * \nabla \partial_{t} g=g^{-1} * \nabla\left(g^{-1} * \mathrm{Rm}+(\nabla \phi)^{* 2}+\nabla^{2} \phi\right) \\
& =g^{*-2} * \nabla \mathrm{Rm}+g^{-1} * \nabla \phi * \nabla^{2} \phi+g^{-1} * \nabla^{3} \phi
\end{aligned}
$$

it follows that

$$
\begin{aligned}
\partial_{t} \nabla \mathrm{Rm}= & \nabla \Delta \mathrm{Rm}+g^{*-2} * \mathrm{Rm} * \nabla \mathrm{Rm}+\nabla^{2} \phi * \nabla^{3} \phi+g^{-1} * \nabla^{2} \mathrm{Rm} * \nabla \phi \\
& +g^{-1} * \nabla \mathrm{Rm} * \nabla^{2} \phi+g^{-1} * \mathrm{Rm} * \nabla^{3} \phi+g^{-1} * \mathrm{Rm} * \nabla \phi * \nabla^{2} \phi \\
= & \Delta \nabla \mathrm{Rm}+g^{*-2} * \mathrm{Rm} * \nabla \mathrm{Rm}+\nabla^{2} \phi * \nabla^{3} \phi+g^{-1} * \mathrm{Rm} * \nabla^{3} \phi \\
& +g^{-1} * \mathrm{Rm} * \nabla \phi * \nabla^{2} \phi+g^{-1} * \nabla \mathrm{Rm} * \nabla^{2} \phi+g^{-1} * \nabla^{2} \mathrm{Rm} * \nabla \phi .
\end{aligned}
$$

From

$$
\begin{aligned}
\partial_{t}|\nabla \mathrm{Rm}|^{2} & =2\left\langle\nabla \mathrm{Rm}, \partial_{t} \nabla \mathrm{Rm}\right\rangle+g^{*-4} * \partial_{t} g^{-1} *(\nabla \mathrm{Rm})^{* 2} \\
& =2\left\langle\nabla \mathrm{Rm}, \partial_{t} \nabla \mathrm{Rm}\right\rangle+g^{-6} *\left(g^{-1} * \mathrm{Rm}+(\nabla \phi)^{2}+\nabla^{2} \phi\right) *(\nabla \mathrm{Rm})^{* 2}
\end{aligned}
$$

we conclude that

$$
\begin{aligned}
\partial_{t}|\nabla \mathrm{Rm}|^{2}= & \Delta|\nabla \mathrm{Rm}|^{2}-2\left|\nabla^{2} \mathrm{Rm}\right|^{2}+g^{*-7} * \mathrm{Rm} *(\nabla \mathrm{Rm})^{* 2} \\
& +g^{*-5} * \nabla \mathrm{Rm} * \nabla^{2} \phi * \nabla^{3} \phi+g^{*-6} * \mathrm{Rm} * \nabla \mathrm{Rm} * \nabla \phi * \nabla^{2} \phi \\
& +g^{*-6} *(\nabla \mathrm{Rm})^{* 2} * \nabla^{2} \phi+g^{*-6} * \nabla \mathrm{Rm} * \nabla^{2} \mathrm{Rm} * \nabla \phi \\
& +g^{*-6} *(\nabla \mathrm{Rm})^{* 2} *(\nabla \phi)^{* 2}+g^{*-6} * \mathrm{Rm} * \nabla \mathrm{Rm} * \nabla^{3} \phi .
\end{aligned}
$$

Consequently,

$$
\partial_{t}|\nabla \mathrm{Rm}|^{2} \leq \Delta|\nabla \mathrm{Rm}|^{2}-2\left|\nabla^{2} \mathrm{Rm}\right|^{2}+C_{2}|\nabla \mathrm{Rm}|\left|\nabla^{2} \mathrm{Rm}\right|+C_{2}|\nabla \mathrm{Rm}|\left|\nabla^{3} \phi\right|
$$

On the other hand, Lemma 2.7 yields

$$
\begin{aligned}
\partial_{t} \nabla^{2} \phi= & \Delta \nabla^{2} \phi+g^{*-2} * \operatorname{Rm} * \nabla^{2} \phi+\beta_{2} \nabla^{2} \phi+|\nabla \phi|^{2} \nabla^{2} \phi \\
& +\nabla \phi * \nabla^{3} \phi+g^{-1} *\left(\nabla^{2} \phi\right)^{* 2}+g^{*-2} * \operatorname{Rm} *(\nabla \phi)^{* 2} .
\end{aligned}
$$

Plugging 3.146 into $\partial_{t}\left|\nabla^{2} \phi\right|^{2}=2\left\langle\nabla^{2} \phi, \partial_{t} \nabla^{2} \phi\right\rangle+g^{*-3} * \partial_{t} g *\left(\nabla^{2} \phi\right)^{* 2}$, we arrive at

$$
\begin{aligned}
\partial_{t}\left|\nabla^{2} \phi\right|^{2}= & \Delta\left|\nabla^{2} \phi\right|^{2}-2\left|\nabla^{3} \phi\right|^{2}+g^{*-4} * \mathrm{Rm} *\left(\nabla^{2} \phi\right)^{* 2}+g^{*-2} *\left(\nabla^{2} \phi\right)^{* 2} \\
& +g^{*-3} *(\nabla \phi)^{* 2} *\left(\nabla^{2} \phi\right)^{* 2}+g^{*-2} * \nabla \phi * \nabla^{2} \phi * \nabla^{3} \phi \\
& +g^{*-3} *\left(\nabla^{2} \phi\right)^{* 3}+g^{*-4} * \mathrm{Rm} *(\nabla \phi)^{* 2} * \nabla^{2} \phi .
\end{aligned}
$$

Consequently,

$$
\partial_{t}\left|\nabla^{2} \phi\right|^{2} \leq \Delta\left|\nabla^{2} \phi\right|^{2}-2\left|\nabla^{3} \phi\right|^{2}+C_{3}\left|\nabla^{3} \phi\right|+C_{3} .
$$

Combining (3.145) and (3.147), we have

$$
\partial_{t}|\nabla \boldsymbol{\Lambda}|^{2} \leq \Delta|\nabla \boldsymbol{\Lambda}|^{2}-\frac{3}{2}\left|\nabla^{2} \boldsymbol{\Lambda}\right|^{2}+C_{4}|\nabla \boldsymbol{\Lambda}|^{2}+C_{4} .
$$

According to Lemma 2.6 and (3.144), for any given positive number $a$, we get

$$
\partial_{t}\left(a+|\boldsymbol{\Lambda}|^{2}\right) \leq \Delta\left(a+|\boldsymbol{\Lambda}|^{2}\right)-\frac{3}{2}|\nabla \boldsymbol{\Lambda}|^{2}+C_{5} .
$$


Therefore

$$
\begin{aligned}
\partial_{t}\left[\left(a+|\boldsymbol{\Lambda}|^{2}\right)|\nabla \boldsymbol{\Lambda}|^{2}\right] \leq & \Delta\left[\left(a+|\boldsymbol{\Lambda}|^{2}\right)|\nabla \boldsymbol{\Lambda}|^{2}\right]-2\left\langle\nabla|\boldsymbol{\Lambda}|^{2}, \nabla|\nabla \boldsymbol{\Lambda}|^{2}\right\rangle \\
& -\frac{3}{2}|\nabla \boldsymbol{\Lambda}|^{4}+C_{5}|\nabla \boldsymbol{\Lambda}|^{2}-\frac{3}{2}\left(a+|\boldsymbol{\Lambda}|^{2}\right)\left|\nabla^{2} \boldsymbol{\Lambda}\right|^{2} \\
& +C_{4}\left(a+|\boldsymbol{\Lambda}|^{2}\right)|\nabla \boldsymbol{\Lambda}|^{2}+C_{4}\left(a+|\boldsymbol{\Lambda}|^{2}\right) .
\end{aligned}
$$

By the definition, the second term on the right-hand side of 3.150 is bounded from above by

$$
\begin{aligned}
& g^{p q} \nabla_{p}\left(|\mathrm{Rm}|^{2}+|\nabla \phi|^{2}\right) \nabla_{q}\left(|\nabla \mathrm{Rm}|^{2}+\left|\nabla^{2} \phi\right|^{2}\right) \\
= & g^{-1} *\left(g^{-4} * \mathrm{Rm} * \nabla \mathrm{Rm}+g^{-1} * \nabla \phi * \nabla^{2} \phi\right) \\
& *\left(g^{*-5} * \nabla \mathrm{Rm} * \nabla^{2} \mathrm{Rm}+g^{*-2} * \nabla^{2} \phi * \nabla^{3} \phi\right) \\
\leq & C_{6}|\nabla \mathrm{Rm}|^{2}\left|\nabla^{2} \mathrm{Rm}\right|+C_{6}|\nabla \mathrm{Rm}|\left|\nabla^{2} \mathrm{Rm}\right|+C_{6}|\nabla \mathrm{Rm}|\left|\nabla^{3} \phi\right|+C_{6}\left|\nabla^{3} \phi\right| \\
\leq & C_{6}|\nabla \boldsymbol{\Lambda}|^{2}\left|\nabla^{2} \boldsymbol{\Lambda}\right|+C_{6}|\nabla \boldsymbol{\Lambda}|\left|\nabla^{2} \boldsymbol{\Lambda}\right|+C_{6}|\nabla \boldsymbol{\Lambda}|\left|\nabla^{2} \boldsymbol{\Lambda}\right|+C_{6}\left|\nabla^{2} \boldsymbol{\Lambda}\right| \\
\leq & \frac{3}{8} a\left|\nabla^{2} \boldsymbol{\Lambda}\right|^{2}+\frac{2 C_{6}^{2}}{3 a}|\nabla \boldsymbol{\Lambda}|^{4}+\frac{6}{8} a\left|\nabla^{2} \boldsymbol{\Lambda}\right|^{2}+\frac{4 C_{6}^{2}}{3 a}|\nabla \boldsymbol{\Lambda}|^{2}+\frac{3}{8} a\left|\nabla^{2} \boldsymbol{\Lambda}\right|^{2}+\frac{2 C_{6}^{2}}{3 a} \\
\leq & \frac{3}{2} a\left|\nabla^{2} \boldsymbol{\Lambda}\right|^{2}+\frac{2 C_{6}^{2}}{a}|\nabla \boldsymbol{\Lambda}|^{4}+\frac{2 C_{6}^{2}}{a}
\end{aligned}
$$

where we used the inequality $x^{2} \leq x^{4}+1$ for any $x \geq 0$. Substituting this inequality into (3.150) implies

$$
\begin{array}{ll}
\partial_{t}\left[\left(a+|\boldsymbol{\Lambda}|^{2}\right)|\nabla \boldsymbol{\Lambda}|^{2}\right] \leq & \Delta\left[\left(a+|\boldsymbol{\Lambda}|^{2}\right)|\nabla \boldsymbol{\Lambda}|^{2}\right]-\left(\frac{3}{2}-\frac{2 C_{6}^{2}}{a}\right)|\nabla \boldsymbol{\Lambda}|^{4}+C_{5}|\nabla \boldsymbol{\Lambda}|^{2} \\
(3.151) & +C_{4}\left(a+|\boldsymbol{\Lambda}|^{2}\right)|\nabla \boldsymbol{\Lambda}|^{2}+C_{4}\left(a+|\boldsymbol{\Lambda}|^{2}\right)+\frac{2 C_{6}^{2}}{a} .
\end{array}
$$

By 3.139 , we can choose $a$ so that

$$
a \geq 4 C_{6}^{2}, \quad a \geq \max _{M \times[0, T]}\left(|\mathrm{Rm}|^{2}+\left|\nabla^{2} \phi\right|^{2}\right) ;
$$

then $\frac{3}{2}-\frac{2 C_{6}^{2}}{a} \geq 1$ and $a \leq a+|\boldsymbol{\Lambda}|^{2} \leq 2 a$. Consequently, we can deduce from 3.151 that

$$
\begin{aligned}
\partial_{t}\left[\left(a+|\boldsymbol{\Lambda}|^{2}\right)|\nabla \boldsymbol{\Lambda}|^{2}\right] \leq & \Delta\left[\left(a+|\boldsymbol{\Lambda}|^{2}\right)|\nabla \boldsymbol{\Lambda}|^{2}\right]-|\nabla \boldsymbol{\Lambda}|^{4} \\
& +\left(C_{4}+\frac{C_{4}}{a}\right)\left(a+|\boldsymbol{\Lambda}|^{2}\right)|\nabla \boldsymbol{\Lambda}|^{2}+2 C_{4} a+\frac{2 C_{6}^{2}}{a} \\
\leq & \Delta\left[\left(a+|\boldsymbol{\Lambda}|^{2}\right)|\nabla \boldsymbol{\Lambda}|^{2}\right]-\frac{1}{4 a^{2}}\left[\left(a+|\boldsymbol{\Lambda}|^{2}\right)|\nabla \boldsymbol{\Lambda}|^{2}\right]^{2} \\
& +\left(C_{4}+\frac{C_{4}}{a}\right)\left(a+|\boldsymbol{\Lambda}|^{2}\right)|\nabla \boldsymbol{\Lambda}|^{2}+2 C_{4} a+\frac{2 C_{6}^{2}}{a} \\
\leq & \Delta\left[\left(a+|\boldsymbol{\Lambda}|^{2}\right)|\nabla \boldsymbol{\Lambda}|^{2}\right]-\frac{1}{8 a^{2}}\left[\left(a+|\boldsymbol{\Lambda}|^{2}\right)|\nabla \boldsymbol{\Lambda}|^{2}\right]^{2}+C_{7} .
\end{aligned}
$$

Consider the function

$$
u:=\left(a+|\boldsymbol{\Lambda}|^{2}\right)|\nabla \boldsymbol{\Lambda}|^{2} t
$$


defined on $M \times[0, T]$. Then

$$
\begin{aligned}
u & =0 \text { on } M \times\{0\}, \\
\partial_{t} u & \leq \Delta u-\frac{1}{8 a^{2} t} u^{2}+C_{7} t+\frac{1}{t} u \quad \text { on } M \times[0, T] .
\end{aligned}
$$

Fix a point $x_{0} \in M$, consider the cutoff function $\xi(x)$ on $M$, introduced in [26], such that

$$
\begin{aligned}
\xi & =1 \text { on } B_{\tilde{g}}\left(x_{0}, 1\right), \quad \xi=0 \quad \text { on } M \backslash B_{\tilde{g}}\left(x_{0}, 2\right), \quad 0 \leq \xi \leq 1, \\
|\tilde{\nabla} \xi|_{\tilde{g}}^{2} & \leq 16 \xi, \quad \tilde{\nabla}^{2} \xi \geq-c \tilde{g}, \quad \text { on } M,
\end{aligned}
$$

where $c$ depends on $k_{0}$. As in [26], we define

$$
F:=\xi u
$$

on $M \times[0, T]$. Then from the definition, we have

$$
F=0 \text { on } M \times\{0\}, \quad F=0 \text { on }\left(M \backslash B_{\tilde{g}}\left(x_{0}, 2\right)\right) \times[0, T], \quad F \geq 0 \text { on } M \times[0, T] .
$$

Without loss of generality, we may assume that $F$ is not identically zero on $M \times$ $[0, T]$. In this case, we can find a point $\left(x_{1}, t_{1}\right) \in B_{\tilde{g}}\left(x_{0}, 2\right) \times[0, T]$ such that

$$
F\left(x_{1}, t_{1}\right)=\max _{M \times[0, T]} F(x, t)>0,
$$

which implies $t_{1}>0$ and

$$
\partial_{t} F\left(x_{1}, t_{1}\right)>0, \quad \nabla F\left(x_{1}, t_{1}\right)=0, \quad \Delta F\left(x_{1}, t_{1}\right) \leq 0 .
$$

If $u\left(x_{1}, t_{1}\right) \leq 1$, then $F\left(x_{1}, t_{1}\right) \leq 1$ by 3.155 and hence

$$
a t|\nabla \boldsymbol{\Lambda}|^{2} \leq u=F \leq 1 \quad \text { on } B_{\tilde{g}}\left(x_{0}, 1\right) \times[0, T] ;
$$

in particular,

$$
|\nabla \mathrm{Rm}|^{2}+\left|\nabla^{2} \phi\right|^{2} \lesssim \frac{1}{t} \quad \text { on } M \times[0, T] .
$$

In the following we assume that $u\left(x_{1}, t_{1}\right) \geq 1$. Under this assumption, together with (3.154) and $\xi \partial_{t} u \geq 0$ at $\left(x_{1}, t_{1}\right)$, we arrive at, at the point $\left(x_{1}, t_{1}\right)$,

$$
\begin{aligned}
0 & \leq \xi \partial_{t} u \leq \xi \Delta u+\left(\frac{1}{t} u+C_{7} t-\frac{1}{8 a^{2} t} u^{2}\right) \xi \\
& \leq \xi \Delta u+\left(\frac{1}{t} u+C_{7} t u-\frac{1}{8 a^{2} t} u^{2}\right) \xi \\
& \leq \xi \Delta u+\frac{u}{t}\left(1+C_{7} t^{2}-\frac{1}{8 a^{2}} u\right) \xi \leq \xi \Delta u+\frac{u}{t}\left(C_{8}-C_{9} u\right) \xi
\end{aligned}
$$

thus

$$
\xi \Delta u+\frac{\xi u}{t}\left(C_{8}-C_{9} u\right) \geq 0 \quad \text { at }\left(x_{1}, t_{1}\right) .
$$

By the same argument in [26] (equations (28)-(35) in page 293), we find that

$$
\frac{\xi u}{t}\left(C_{9} u-C_{8}\right) \leq C_{10} u-u \Delta \xi \quad \text { at }\left(x_{1}, t_{1}\right) .
$$

According to the equation (38) in page 294 of [26], we get

$$
-\Delta \xi \leq C_{11}+g^{\alpha \beta}\left(\Gamma_{\alpha \beta}^{\gamma}-\tilde{\Gamma}_{\alpha \beta}^{\gamma}\right) \tilde{\nabla}_{\gamma} \xi .
$$

Since

$$
\partial_{t} \Gamma=g^{*-2} * \nabla \mathrm{Rm}+g^{-1} * \nabla^{3} \phi+g^{-1} * \nabla \phi * \nabla^{2} \phi,
$$


it follows that

$$
\left|\partial_{t} \Gamma\right| \leq C_{11}|\nabla \mathbf{\Lambda}|+C_{12} \leq \frac{C_{11}}{\sqrt{a t}} u^{1 / 2}+C_{12}
$$

Since $\xi\left(x_{1}\right) u\left(x_{1}, t\right)=F\left(x_{1}, t\right) \leq F\left(x_{1}, t_{1}\right)$ for $t \in[0, T]$, we obtain

$$
\left|\partial_{t}\left(x_{1}, t\right)\right| \leq C_{11}\left[\frac{F\left(x_{1}, t_{1}\right)}{a \xi\left(x_{1}\right)}\right]^{1 / 2} \frac{1}{\sqrt{t}}+C_{12}
$$

for any $t \in[0, T]$. As showed in [26] (the equation (45) in page 295), together with $|\tilde{\nabla} \xi|_{\tilde{g}} \leq 4 \xi^{1 / 2}$, we find that

$$
g^{\alpha \beta}\left(\Gamma_{\alpha \beta}^{\gamma}-\tilde{\Gamma}_{\alpha \beta}^{\gamma}\right) \tilde{\nabla}_{\gamma} \xi \leq C_{13} F\left(x_{1}, t_{1}\right)^{1 / 2}+C_{14} ;
$$

substituting 3.159 into 3.158$)$ implies

$$
-\Delta \xi \leq C_{15}+C_{13} F\left(X_{1}, t_{1}\right)^{1 / 2} \text { at }\left(x_{1}, t_{1}\right) .
$$

According to (3.157) and (3.160), we have the following inequality

$$
\xi u\left(C_{9} u-C_{8}\right) \leq C_{10} t u+C_{15} t u+C_{13} t u F^{1 / 2} \leq C_{16} u+C_{16} u F^{1 / 2} \text { at }\left(x_{1}, t_{1}\right) ;
$$

multiplying by $\xi\left(x_{1}\right)$ yields

$$
C_{9} F^{2} \leq C_{17} F+C_{16} F^{3 / 2} \text { at }\left(x_{1}, t_{1}\right),
$$

from which we deduce that $F\left(x_{1}, t_{1}\right) \lesssim 1$ and therefore

$$
\xi u \lesssim 1 \quad \text { on } M \times[0, T] .
$$

In particular, $u \lesssim 1$ on $M \times[0, T]$ since $x_{0}$ was arbitrary. From the definition (3.152), this tells us the estimate $|\nabla \boldsymbol{\Lambda}|^{2} \lesssim 1 / t$ on $M \times[0, T]$, where $\lesssim$ depends only on $m, k_{0}, k_{1}, k_{2}, \alpha_{1}, \alpha 2, \beta_{1}, \beta_{2}$. Hence the lemma holds for $n=1$.

By induction, suppose for $s=1, \cdots, n-1$ we have

$$
\left|\nabla^{s} \mathrm{Rm}\right|^{2}+\left|\nabla^{s+2} \phi\right|^{2} \lesssim \frac{1}{t^{s}}
$$

on $M \times[0, T]$. As in [26], we define a function

$$
v:=\left(a+t^{n-1}\left|\nabla^{n-1} \boldsymbol{\Lambda}\right|^{2}\right)\left|\nabla^{n} \boldsymbol{\Lambda}\right|^{2} t^{n}
$$

and choose $a$ sufficiently large. Similarly to 3.154 we can show that

$$
\partial_{t} v \leq \Delta v-\frac{C_{18}}{a^{2} t} v^{2}+C_{19}+\frac{C_{20}}{t} v
$$

on $M \times[0, T]$. Using the same cutoff function $\xi$ and arguing in the same way, we obtain that $v \lesssim 1$ on $M \times[0, T]$. Hence the inequality (3.140) holds for $s=n$.

\section{REFERENCES}

[1] Brendle, Simon. Uniqueness of gradient Ricci solitons, Math. Res. Lett., 18(2011), no. 3, 531-538. MR2802586 (2012e: 53073)

[2] Brendle, Simon. Rotational symmetry of self-similar solutions to the Ricci flow, Invent. Math., 194(2013), no. 3, 731-764. MR3127066

[3] Brendel, Simon.Rotational symmetry of Ricci solitons in higher dimensions, J. Differential Geom., 97(2014), no. 2, 191-214. MR3231974

[4] Cao, Huai-Dong. Existence of gradient Kähler-Ricci solitons. Elliptic and parabolic methods in geometry (Minneapolis, MN, 1994), 1-16, AK Peters, Wellesley, MA, 1996. MR1417944 (98a: 53058)

[5] Cao, Huai-Dong. Recent progress on Ricci solitons. Recent adcances in geometry analysis, 1-38, Adv. Lect. Math. (ALM), 11, Int. Press, Someville, MA, 2010. MR2648937 (2011d: $53061)$ 
[6] Cao, Huai-dong; Chen, Qiang. On locally conformally flat gradient steady Ricci solitons, Trans. Amer. Math. Soc., 364(2012), no. 5, 2377-2391. MR2888210

[7] Catino, Giovanni; Mantegazza, Carlo. Evolution of the Weyl tensor under the Ricci flow, Ann. Inst. Fourier (Grenoble), 61(2011), no. 4, 1407-1435. MR2951497

[8] Chen, Bing-Long. Strong uniqueness of the Ricci flow, J. Differential Geom., 82(2009), no. 2, 363-382. MR25207960 (2019h: 53095)

[9] Chow, Bennett; Lu, Peng; Ni, Lei. Hamilton's Ricci flow. Graduate Studies in Mathematics, 77. American Mathematical Society, Providence, RI; Science Press, New York, 2006. xxxv+608 pp. MR2274812 (2008a: 53068)

[10] Chow, Bennett; Lu, Peng; Yang, Bo. Lower bounds for the scalar curvatures of noncompact gradient Ricci solitons, C. R.Math. Acad. Sci. Paris, 349(2011), no. 23-24, 1265-1267. MR2861997

[11] Chow Bennett; Chu Sun-Chin; Glickenstein David; Guenther Christine; Isenberg James; Ivey Tom; Knopf Dan; Lu Peng; Luo Feng; Ni Lei. The Ricci flow: Techniques and Applications. Part I. Geometric aspects, Mathematical Surveys and Monographs, 135, American Mathematical Society, Providence, RI, 2007. xxiv+536 pp. MR2302600 (2008 f: 53088)

[12] Fernández-López, Manuel; García-Río, Eduardo. A sharp lower bound for the scalar curvature of certain steady gradient Ricci solitons, Proc. Amer. Math. Soc., 141(2013), no. 6, 21452148. MR3034440

[13] Hamilton, Richard S. Three-manifolds with positive Ricci curvature, J. Differential Geom., 17(1982), no. 2, 255-306. MR0664497 (84a: 53050)

[14] He, Chun-Lei; Hu, Sen; Kong, De-Xing; Liu, kefeng. Generalized Ricci flow I: Local existence and uniqueness. Topology and physis, 151-171, Nankai Tracts Math., 12, World Sci. Publ., Hackensack, NJ, 2008. MR2503395 (2010k: 53098)

[15] Hamilton, Richard S. The formation of singularities in the Ricci flow. Surveys in differential geometry, Vol. II (Cambridge, MA, 1993), 7-136, Int. Press, Cambridge, MA, 1995. MR1375255 (97e: 53075)

[16] Ivey, Thomas. New examples of complete Ricci solitons, Proc. Amer. Math. Soc., 122(1994), no. 1, 241-245. MR1207538 (94k: 53057)

[17] Li, Yi. Generalized Ricci flow I: higher derivatives estimates for compact manifolds, Analysis \& PDE., 5(2012), no. 4, 747-775. MR3006641

[18] Li, Yi. Eigenvalues and entropies under the harmonic-Ricci flow, Pacific J. Math., 267(2014), no. 1, 141-184. MR3163480

[19] List, B. Evolution of an extended Ricci flow system, PhD thesis. AEI Postdam, 2005.

[20] Müler, Reto. Monotone volume formulas for geometric flow, J. Reine Angew. Math., 643(2010), 39-57. MR2658189 (2011k: 53086)

[21] Müler, Reto. Ricci flow coupled with harmonic map flow, Ann. Sci. Éc. Norm. Supér. (4) 45 (2012), no. 1, 101-142. MR2961788.

[22] Perelman, Grisha. The entropy formula for the Ricci flow and its geometric applications, arXiv: math/0211159

[23] Perelman, Grisha. Ricci flow with surgery on three-manifolds, arXiv: math/0303109

[24] Perelman, Grisha. Finite extinction time for the solutions to the Ricci flow on certain threemanifolds, arXiv: math/0307245

[25] Petersen, Peter; Wylie, William. Rigidity of gradient Ricci solitons, Pacific J. Math., 241(2009), no. 2, 329-345. MR2507581 (2010j: 53071)

[26] Shi, Wan-Xiong. Deforming the metric on complete Riemannian manifolds, J. Differential Geom., 30(1989), no. 1, 223-301. MR1001277 (90i: 58202)

[27] $\mathrm{Hu}, \mathrm{Xue}$; Shi, YuGang. Static flow on complete noncompact manifolds I: short-time existence and asymptotic expansions at conformal infinity, Sci. China Math., 55(2012), no. 9, 18831900. MR2960867

[28] Yau, Shing-Tung. Harmonic functions on complete Riemannian manifolds, Comm. Pure Appl. Math., 28(1975), 201-228. MR0431040 (55\# 4042).

[29] Yau, Shing-Tung. On the Ricci curvature of a compact Kähler manifold and the complex Monge-Ampère equation. I., Comm. Pure Appl. Math., 31(1978), no. 3, 339-411. MR0480350 (81d: 53045). 
Mathematics Research Unit, FStC, University of Luxembourg, 6 rue Richard CoudenhoveKalergi, L-1359 Luxembourg, Grand-Duchy of Luxembourg

E-mail address: yilicms@gmail.com 